CHAPTER 5

\title{
Optical Properties of Excitons in ZnSe-based Quantum Well Heterostructures
}

\author{
Roberto Cingolani \\ Dipartimento di Scienza dei Materiali \\ UNIVERSTÍ Á DI LECCE \\ LECCE, ITALY
}

I. INTRODUCTION . . . . . . . . . . . . . . . . . . . . . . . . . . . . . . . 163

II. Modeling of Excitonic States In II-VI Quantum Wells ． . . . . . . . 164

III. Linear Optical Properties of QUASI-TWO-DIMENSIONAL ExcItons . . . . . . 169

1. Optical Absorption . . . . . . . . . . . . . . . . . . . . . . . . . . . . . . . . 169

2. Excitons at the Dimensionality Cross-over . . . . . . . . . . . . . . . 185

3. Phototransport Processes . . . . . . . . . . . . . . . . . . . . . . 189

4. Temporal Evolution of the Excitonic Transitions . . . . . . . . . . . . . 195

IV. Nontinear EXCITONIC Properties . . . . . . . . . . . . . . . . . . . . 202

1. Basic Theoretical Concepts . . . . . . . . . . . . . . . . . . . . . . 202

2. Excitons and the One-component Electron Plasma . . . . . . . . . . . 205

3. Excitons and the Electron-hole Plasma . . . . . . . . . . . . . . . . . 211

V. Role of Excitons in THE Lasing Of ZnSe-Based Quantum Wells . . . . . . 215

VI. ConClusions . . . . . . . . . . . . . . . . . . . . . . . . . . . . . . 223

REFERENCES . . . . . . . . . . . . . . . . . . . . . 223

\section{Introduction}

Excitons in ZnSe-based quantum well heterostructures exhibit strong stability as compared to bulk semiconductors or III-V quantum wells, due to the enhancement of the binding energy and the reduction of the exciton-phonon coupling caused by quantum confinement. Due to these effects excitons are expected to play an important role in many-body processes such as lasing and nonlinear absorption of II-VI quantum wells even at room temperature. For example, excitonic gain has been demonstrated in $\mathrm{Zn}_{1-x} \mathrm{Cd}_{x} \mathrm{Se} / \mathrm{ZnSe}$ quantum wells of thickness and composition such that the exciton binding energy was larger than the LO phonon energy $\left(E_{b}>\hbar \omega_{\mathrm{LO}}\right)$ (Ding et al., 1992). A detailed study of excitons in ZnSe multiple quantum wells (MQWs) is thus important to understand the optical 
properties of these wide gap heterostructures, also in view of their application to blue-green optoelectronic devices. In this chapter we overview the most recent experimental and theoretical work about the optical properties of excitons in ZnSe-based quantum wells. In Section II we briefly summarize the basic theoretical concepts used to model quasi-two-dimensional excitons in quantum wells. In Section III, we discuss the linear optical properties of excitons, including the electrostatic and thermal stability, the strength of the quantum size effect in quantum wells and thin films, the phototransport properties and the temporal evolution of the excitonic transitions. In Section IV we treat the nonlinear optical properties of excitons. After a short theoretical introduction, we discuss the interaction of excitons with a single-carrier and a two-carrier plasma. Finally, we discuss the role of excitons in the lasing processes of II-VI quantum wells in Section V. Our conclusions are drawn in Section VI.

\section{Modeling of Excitonic States in II-VI Quantum Wells}

We concentrate on II-VI quantum wells consisting of binary compounds $\mathrm{ZnSe}, \mathrm{CdSe}, \mathrm{ZnS}$ and their solid solutions, $\mathrm{ZnCdSe}, \mathrm{ZnSSe}$. The combination of these materials spans the whole green-blue spectral range (Fig. 1), though with the complication of lattice mismatch which strongly affects the electronic states and the valence band offset.

The shift of the conduction (c), heavy-hole (HH), and light-hole (LH) band edges due to the hydrostatic and uniaxial deformation of the strained semiconductor is given by (Pollak and Cardona, 1968):

$$
\begin{aligned}
\delta E_{c} & =2 \cdot \alpha_{c} \cdot\left(\frac{C_{11}-C_{12}}{C_{11}}\right) \cdot \sigma \\
\delta E_{H H} & =\left(-2 \cdot \alpha_{v} \cdot\left(\frac{C_{11}-C_{12}}{C_{11}}\right)+b \cdot\left(\frac{C_{11}+2 C_{12}}{C_{11}}\right)\right) \cdot \sigma \\
\delta E_{L H} & =\left(-2 \cdot \alpha_{v} \cdot\left(\frac{C_{11}-C_{12}}{C_{11}}\right)-\frac{1}{2} b \cdot\left(\frac{C_{11}+2 C_{12}}{C_{11}}\right)\right) \cdot \sigma \\
- & \frac{\Delta}{2}+\frac{1}{2} \cdot \sqrt{\Delta^{2}+\frac{9}{4}\left(2 b \cdot\left(\frac{C_{11}+2 C_{12}}{C_{11}}\right) \cdot \sigma\right)^{2}-2\left(b \cdot\left(\frac{C_{11}+2 C_{12}}{C_{11}}\right) \cdot \sigma\right) \cdot \Delta}
\end{aligned}
$$

where $\alpha_{c}$ and $\alpha_{v}$ are the conduction and valence band hydrostatic deformation potentials, respectively, $C_{i j}$ are the elastic constants, $\sigma$ is the strain, $b$ is 


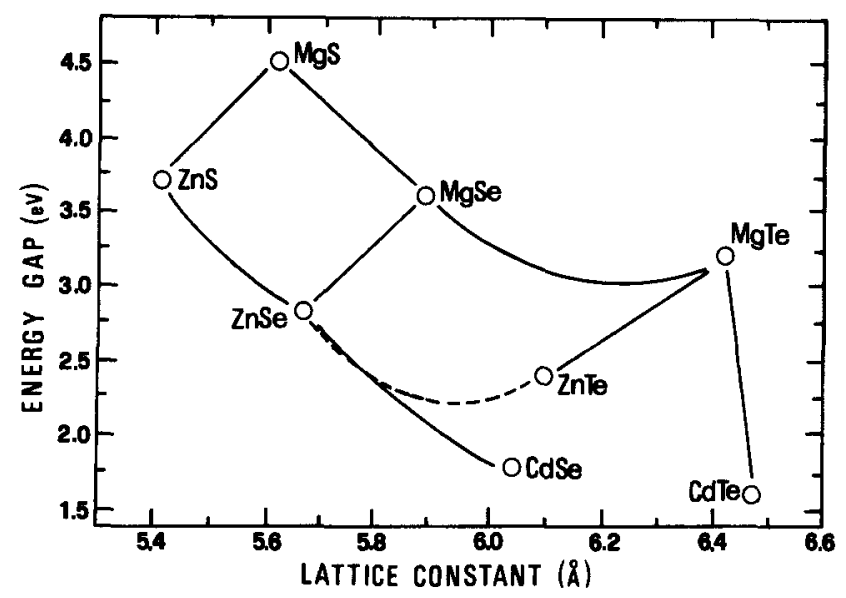

Fig. 1. Energy gap at $300 \mathrm{~K}$ versus lattice constant for a few relevant II-VI semiconductors.

the uniaxial deformation potential, and $\Delta$ the spin orbit splitting. Actually, the way in which the total deformation potential $\alpha$ is shared between the conduction and valence bands $\left(\alpha=\alpha_{c}+\alpha_{v}\right)$ is unknown. This is usually determined empirically, by comparing experimental data and calculations assuming different band-offsets and $\alpha_{c} / \alpha_{c}$ ratio.

The calculation of the confinement energies and excitonic states requires a detailed knowledge of the band structure parameters. Those used in this chapter are summarized in Table I. For the solid solutions, the effective masses and dielectric constants are scaled linearly with the stoichiometric fraction, whereas the band gap is usually scaled taking into account some bowing (Tamargo et al., 1991). The electronic states are evaluated following the usual envelope function model, in the effective mass approximation (Bastard, 1991). The main problem is the conduction to valence band offset ratio assumed in the calculations. There is presently some spread of data in the literature concerning the $\mathrm{ZnSe} / \mathrm{CdSe}$ material system, whereas the negligible conduction band offset of the $\mathrm{ZnS} / \mathrm{ZnSe}$ system is well documented. The available data and their differences will be commented in Section III.1.

The exciton binding energy is a very important parameter to be evaluated in II-VI compounds. The enhancement of the excitonic stability due to quantum confinement and reduced phonon coupling is one of the most interesting properties of II-VI quantum wells for optoelectronics. What is important for technology is the correct description of the well width and composition dependence of the exciton binding energy. This in turn depends 
TABLE 1

Main Structural and Electronic Parameters of Binary II-VI COMpounds

\begin{tabular}{llll}
\hline & \multicolumn{1}{c}{$\mathrm{ZnSe}$} & \multicolumn{1}{c}{$\mathrm{CdSe}$} & $\mathrm{ZnS}$ \\
\hline$E_{g}(e V)$ & $2.821^{a}$ & $1.765^{a}$ & $3.840^{b}$ \\
$m_{e}\left(m_{0}\right)$ & $0.16^{a}$ & $0.13^{a}$ & $0.34^{c}$ \\
$m_{h_{h}}\left(m_{0}\right)$ & $0.6^{a}$ & $0.45^{a}$ & $1.76^{d} / 0.61^{e}$ \\
$m_{l h}\left(m_{0}\right)$ & $0.145^{b}$ & - & - \\
$\varepsilon$ & $8.8^{b}$ & $9.3^{b}$ & $8.32^{b}$ \\
$a(\AA)$ & $5.6676^{a}$ & $6.077^{a}$ & $5.4060^{b}$ \\
$C_{11}\left(N / m^{2}\right)$ & $8.26 \cdot 10^{10 a}$ & $6.67 \cdot 10^{10 a}$ & $10.40 \cdot 10^{10 f}$ \\
$C_{12}\left(N / m^{2}\right)$ & $4.98 \cdot 10^{10 a}$ & $4.63 \cdot 10^{10 a}$ & $6.50 \cdot 10^{10 f}$ \\
$\alpha(\mathrm{eV})^{g}$ & -1.2 & - & $-4.53^{h}$ \\
$b(\mathrm{eV})$ & -1.2 & 0.8 & $-1.25^{i}$ \\
$\Delta(\mathrm{eV})$ & $0.43^{b}$ & - & $0.070^{b}$ \\
\hline
\end{tabular}

The conduction to valence band offsets adopted in the calculations are $80: 20$ for the $\mathrm{ZnCdSe} / \mathrm{ZnSe}$ material system, and about 5:95 for the $\mathrm{ZnSe} / \mathrm{ZnSSe}$ heterostructures, including the effect of strain.

For the ternary alloys, the effective masses and dielectric constant are evaluated by linear interpolation from the bulk values of the corresponding binary compounds. The energy gap is also linearly scaled unless otherwise stated.

${ }^{a}$ Lozykowsky and Shastri, 1991.

'Landolt and Bornstein, 1982.

'Yokogawa et al., 1994.

'Lawaetz, 1971.

'Lippens and Lanoo, 1989.

${ }^{5}$ Singh and Singh, 1987.

${ }^{\theta} \alpha=-\frac{C_{11}+2 C_{12}}{3} \cdot \frac{\partial E_{g}}{\partial P}$, where the $\frac{\partial E_{g}}{\partial P}$ was taken after Thomas et al., 1992.

"Ves et al., 1990.

'Shahzad et al, 1988.

on the offset which is affected by the strain. The exciton binding energy is usually evaluated by a variational calculation. In the effective mass approximation, the in-plane motion of the electron and hole is transformed into a center-of-mass motion of the exciton and in the relative motion of the constituent carriers. Neglecting the kinetic energy term of the in-plane motion, the exciton Hamiltonian can be written as:

$H=\sum_{i=e, h}\left[-\frac{\hbar^{2}}{2 m_{i}} \frac{\partial^{2}}{\partial z_{i}^{2}}+V_{i}\left(z_{i}\right)\right]-\frac{\hbar^{2}}{2 \mu}\left(\frac{\partial^{2}}{\partial x^{2}}+\frac{\partial^{2}}{\partial y^{2}}\right)-\frac{e^{2}}{\varepsilon \sqrt{\rho^{2}+\left(z_{e}-z_{h}\right)^{2}}}$

where $m_{e}, m_{h}$ are the effective masses of the electron and hole, $\mu$ is the reduced exciton mass, $z_{e}$ and $z_{h}$ are the coordinates perpendicular to the plane of the layers for the electron and hole, $\rho$ is the in-plane relative 
position given by $\rho=\sqrt{x^{2}+y^{2}}, x$ and $y$ are the relative coordinates of electrons and holes, $\varepsilon$ is the dielectric constant, $V_{e}\left(z_{e}\right)$ and $V_{h}\left(z_{h}\right)$ are the actual confinement potentials for electrons and holes, respectively. The lowest exciton energy is calculated variationally with a simple trial function

$$
\Psi\left(\rho, z_{e}, z_{h}\right)=\psi_{e}\left(z_{e}\right) \psi_{h}\left(z_{h}\right) \phi_{e-h}(\rho)
$$

where $\psi_{e}\left(z_{e}\right)$ and $\psi_{h}\left(z_{h}\right)$ are the exact electron and hole wavefunctions in the finite quantum well, respectively. $\phi$ is the wavefunction of the in-plane radial motion, given by a $1 S$-orbital

$$
\phi_{e-h}(\rho)=\left[\frac{2}{\pi \lambda^{2}}\right]^{1 / 2} \exp \left[-\frac{\rho}{\lambda}\right]
$$

where $\lambda$ is the trial parameter representing the radius of the exciton orbit. This separable trial function is strictly correct only for narrow well structures (Bastard et al., 1982; Miller et al., 1985), however it can be used to obtain an estimate of the exciton binding energy also for wider wells, its limiting value being the bulk exciton Rydberg.

The two-dimensional exciton radius is determined by maximizing variationally the exciton binding energy

$$
E_{b}(\lambda)=-\frac{\hbar^{2}}{2 \mu \lambda^{2}}+\frac{e^{2}}{4 \pi \varepsilon}\langle\Psi|1 / \rho| \Psi\rangle
$$

The calculated exciton binding energy strongly depends on the degree of confinement of the electronic wavefunctions. Type I quantum wells like $\mathrm{ZnCdSe} / \mathrm{ZnSe}$ exhibit binding energies ranging between the bulk value (about $20 \mathrm{meV}$ ) up to $40 \mathrm{meV}$ or more, depending on the well depth and width (Fig. 2). Conversely, structures with vanishing potential offset, like the $\mathrm{ZnSe} / \mathrm{ZnS}$ MQWs (very small conduction band offset) have bulk-like excitonic properties (Galbraith, 1992). In Section III.1 we will compare the calculated exciton binding energies with the available experimental data.

Another important figure of merit of the ZnSe-based MQWs is the considerable enhancement of the optical absorption strength. For light propagating perpendicular to the basal plane of the quantum well, this is given by (Sugawara, 1992)

$$
\alpha \cdot L_{x} \simeq \frac{\left|P_{c v}\right|^{2}}{E_{b} \lambda^{2}}\left|\left\langle\psi_{e}\left(z_{e}\right) \mid \psi_{h}\left(z_{h}\right)\right\rangle\right|^{2} \cdot L\left(\hbar \omega-E_{b}\right)
$$




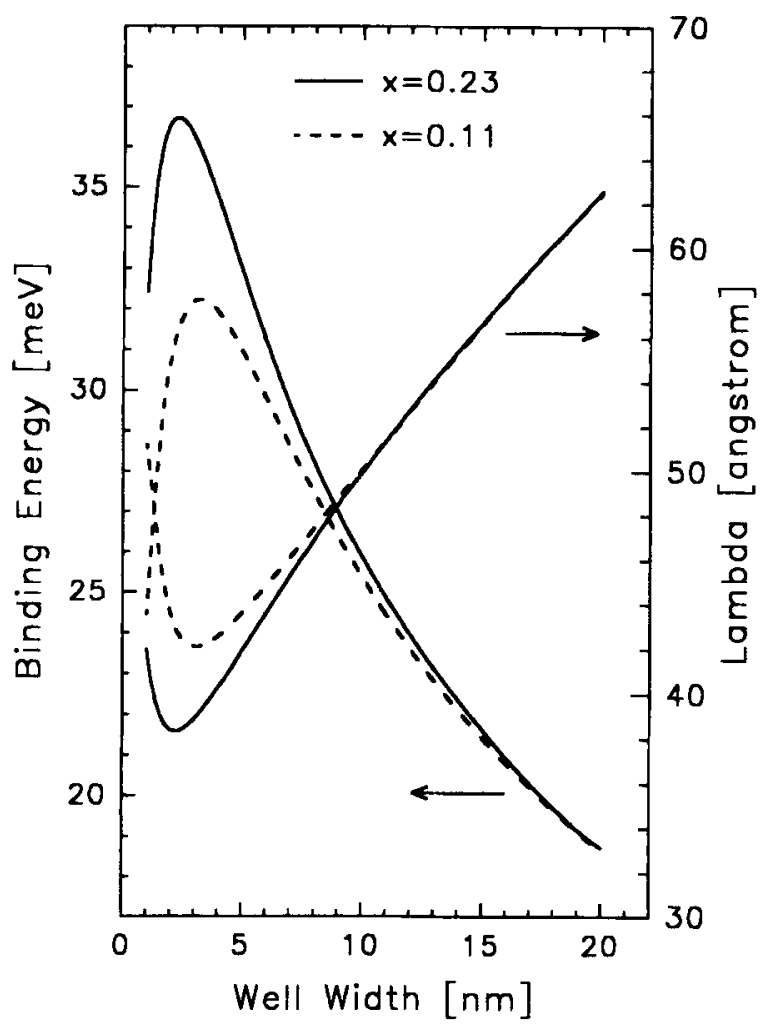

FIg. 2. Variational calculation of the exciton binding energy (left-hand scale) and transverse extent of the exciton wavefunction ( $\lambda$ on the right-hand scale) of $\mathrm{Zn}_{(1-x)} \mathrm{Cd}_{x} \mathrm{Se} / \mathrm{ZnSe}$ quantum wells of different composition.

where $P$ is the momentum matrix element and $L$ is a Lorentzian function modeling the exciton density of states. Calculations performed with available material parameters (Table I) clearly show that the integrated absorption strength is $2-3$ times larger than the corresponding value of $\mathrm{III}-\mathrm{V}$ (GaAs) quantum wells. This finding, together with the extremely large exciton binding energy, suggests that excitons play a very important role in the linear and nonlinear optical response of ZnSe-based quantum wells. It is therefore very important to have a precise description of the excitonic properties, in order to understand some of the relevant optoelectronic phenomena of interest for modern technology: namely, lasing, optical modulation, and nonlinear switching. 


\section{Linear Optical Properties of Quasi-two-dimensional Excitons}

\section{Optical Absorption}

Representative low-temperature absorption spectra of selected $\mathrm{Zn}_{1-x} \mathrm{Cd}_{x^{-}}$ $\mathrm{Se} / \mathrm{ZnSe} \mathrm{MQWs}$ are shown in Figure 3. The samples investigated here were grown by molecular beam epitaxy (Bratina et al., 1993) on GaAs substrates. The width and composition of the $\mathrm{ZnCdSe}$ quantum wells of these samples span the range from bulk-like (3D) behavior to quasi-two-dimensional (2D) limit. Changes in composition $x$ cause variations in the depth of the quantum well and in the value of the elastic strain within the $\mathrm{Zn}_{1-x} \mathrm{Cd}_{x} \mathrm{Se}$ layers, which are all expected to be pseudomorphically strained to the $\mathrm{ZnSe}$ buffer. The combination of these compositional and configurational par-

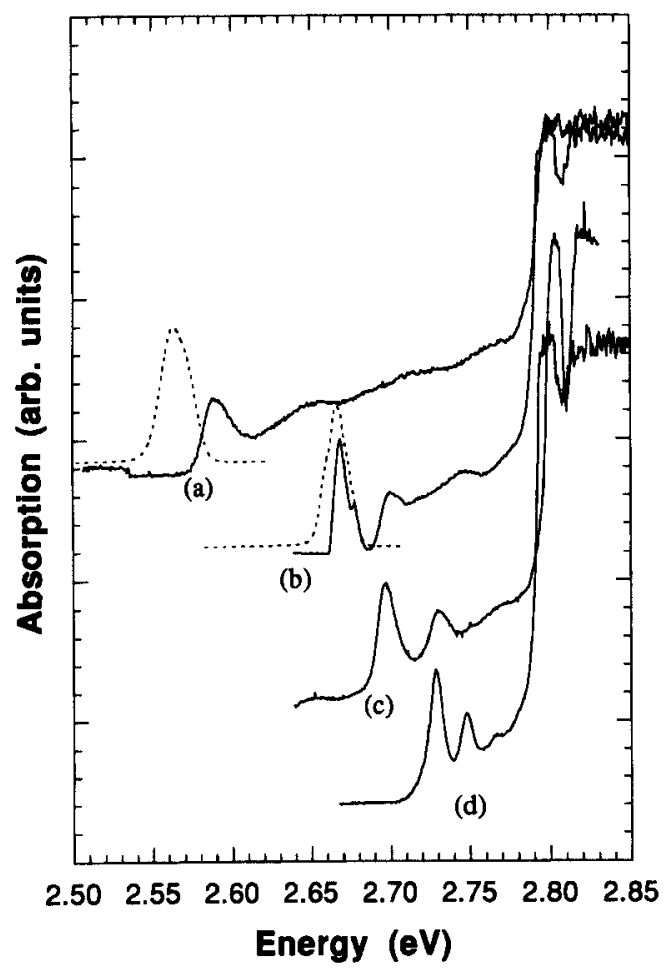

Fig. 3. Absorption (continuous lines) and photoluminescence (dashed lines) spectra of different $\mathrm{Zn}_{(1-x)} \mathrm{Cd}_{x} \mathrm{Se} / \mathrm{ZnSe}$ multiple quantum wells at $10 \mathrm{~K}$. The well width and composition are: (a) $x=0.23, L_{w}=3 \mathrm{~nm}$, (b) $x=0.11, L_{w}=7 \mathrm{~nm}$, (c) $x=0.16, L_{w}=3 \mathrm{~nm}$, (d) $x=0.11$, $L_{w}=3 \mathrm{~nm}$. 
ameters permits a fine tuning of the excitonic properties of the $\mathrm{ZnCdSe}$ MQWs, which can be investigated by systematic optical and structural studies.

For transmission measurements, the heterostructures are glued to a sapphire plate and the GaAs substrate is mechanically polished down to a thickness of about $80 \mu \mathrm{m}$. The samples are then masked with a photoresist leaving the substrate exposed through 100- $\mu$ m-diameter windows, and the $\mathrm{GaAs}$ selectively removed by wet chemical etching using a $\mathrm{H}_{3} \mathrm{PO}_{4}: \mathrm{H}_{2} \mathrm{O}_{2}: \mathrm{H}_{2} \mathrm{O}$ solution at $30^{\circ} \mathrm{C}$ followed by a $\mathrm{H}_{2} \mathrm{SO}_{4}: \mathrm{H}_{2} \mathrm{O}_{2}: \mathrm{H}_{2} \mathrm{O}$ solution at $40^{\circ} \mathrm{C}$. The strong excitonic feature around $2.8 \mathrm{eV}$ is due to the $\mathrm{ZnSe}$ barrier and buffer layer absorption. At lower energies all samples exhibit distinct excitonic peaks superimposed to the QW continuum. The quantum size effect is clearly demonstrated by the blue-shift of the absorption spectrum with decreasing well width. Furthermore, samples with higher $\mathrm{Cd}$ content show absorption spectra extended toward the green range, reflecting the increased depth of the quantum well. Narrow wells $\left(L_{W}=3 \mathrm{~nm}\right)$ exhibit a single confined subband, as expected. The absorption spectra become more structured in the 20-nm-wide MQWs, where at least three electron subbands may be confined in the well. The photoluminescence spectra (dashed lines in Fig. 3) exhibit clear excitonic bands with typical Stokes shift ranging between $2 \mathrm{meV}$ for wide wells with little Cd content, and $20 \mathrm{meV}$ for the narrow wells with high $\mathrm{Cd}$ content. This indicates that the exciton localization at compositional or thickness fluctuations is more important in deep (Cd-rich) quantum wells. X-ray diffraction studies (Cingolani et al., 1994a) show distinct satellite peaks due to the superlattice periodicity, with a relevant broadening of the peaks due to disorder. A typical rocking curve measured in the vicinity of the symmetric (002) direction is shown in Figure 4. The quantitative analysis of these $x$-ray patterns based on the dynamical diffraction theory (Tapfer and Ploog, 1986) provides the well width and composition in good agreement with the nominal parameters. In particular the stoichiometric $\mathrm{Cd}$ content is found to vary by $\pm 1 \%$ around the growth value. A more detailed analysis of the satellite peaks line-shape, which is beyond the scope of this chapter, reveals some inhomogeneous strain fluctuation presumably caused by a smooth long-range modulation of the quantum well thickness having a somewhat "wavy" profile. Both these effects produce relevant potential modulation in the quntum well, causing carrier and/or exciton localization. This localization affects dramatically the optical properties of II-VI quantum wells. We should mention that a quantitative understanding of the microscopic disorder in II-VI quantum wells is still lacking and much work has to be done in order to clarify this important issue. So far there are a number of phenomenological approaches to the disorder-induced broadening of 


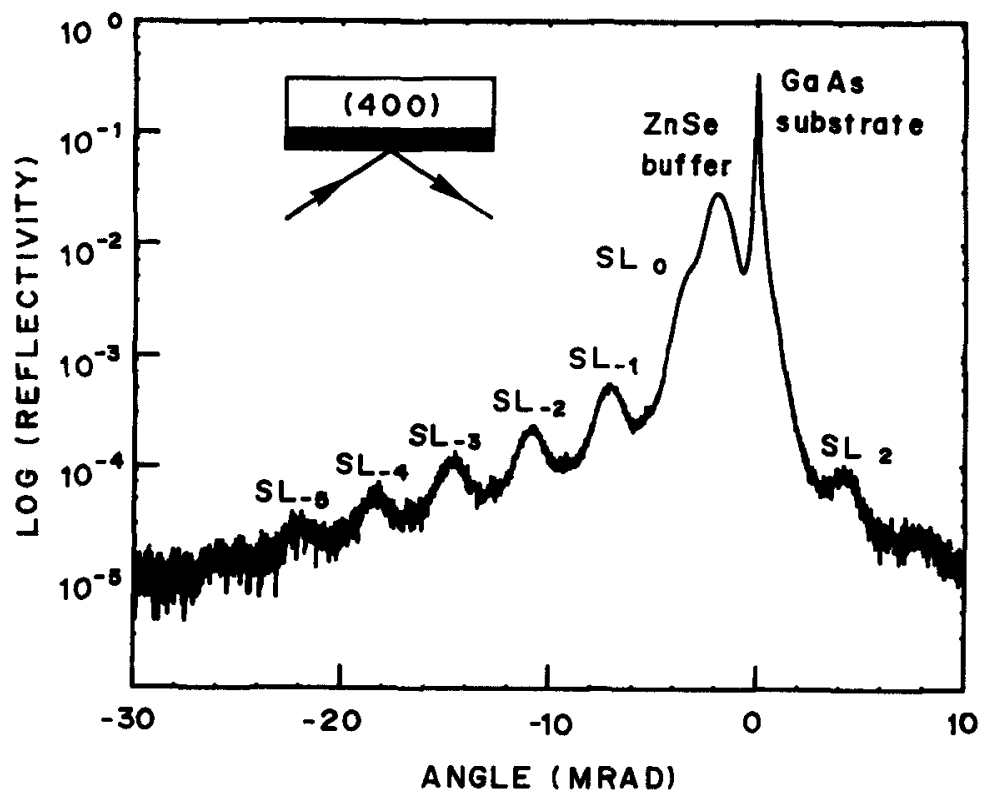

Fig. 4. Double crystal $x$-ray diffraction pattern of a $\mathrm{Zn}_{0.9} \mathrm{Cd}_{0.1} \mathrm{Se} / \mathrm{ZnSe}$ multiple quantum well structure recorded near the (400) reflection of the GaAs, using the $\mathrm{CuK} \alpha_{1}$ radiation. The well width is $2.8 \mathrm{~nm}$, the barrier width is $21.7 \mathrm{~nm}$.

the optical spectra. These are based on a statistical distribution of disorder (either compositional or thickness fluctuation), which is assumed to cause a Gaussian broadening of the single particle states (Young et al., 1994; Baranowskii et al., 1993), usually referred to as inhomogeneous broadening. Furthermore, the formation of ternary alloy at the interface of thin binary/binary quantum wells has been experimentally investigated by Zhu et al. (1993) for the CdSe/ZnSe system and by Yao et al. (1991) for the $\mathrm{ZnS} / \mathrm{ZnSe}$ system.

The temperature dependence of the absorption spectra provides information on the thermal stability of excitons in these quantum wells. In Figure $5 \mathrm{a}$ and $\mathrm{b}$ we show absorption spectra in the $10-300 \mathrm{~K}$ temperature range for MQWs with $x=0.16$ and $L_{W}=3 \mathrm{~nm}$, and $x=0.11$ and $L_{W}=20 \mathrm{~nm}$, respectively. As recently reported by various authors (Ding et al., 1990a; Ding et al., 1992; Ding et al., 1993; Pelekanos et al., 1992a; Cingolani et al., 1995; Liaci et al., 1995, Pellegrini et al., 1995) deep quantum wells (high Cd content) have strongly confined excitons, with large binding energies and clear exciton features observed up to room temperature (Fig. 5a). On the contrary, shallow quantum wells (lower $\mathrm{Cd}$ content) do not exhibit room 

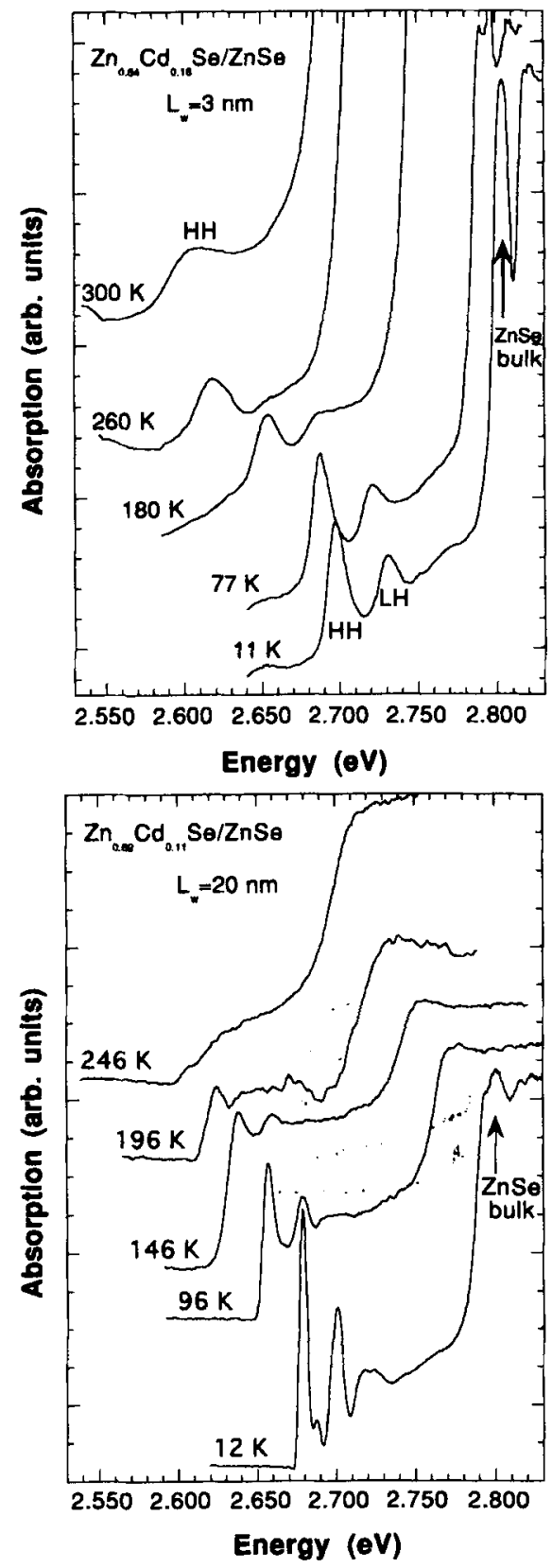

Fig. 5. (a) Temperature-dependent absorption spectra of a $\mathrm{Zn}_{0.84} \mathrm{Cd}_{0.11} \mathrm{Se} / \mathrm{ZnSe}$ multiple quantum well sample with $L_{w}=3 \mathrm{~nm}$. (b) Temperature-dependent absorption spectra of a $\mathrm{Zn}_{0.89} \mathrm{Cd}_{0.11} \mathrm{Se} / \mathrm{ZnSe}$ multiple quantum well sample with $L_{w}=20 \mathrm{~nm}$. 
temperature excitonic absorption (Fig. 5b). The thermal stability of the exciton depends on the ratio of the exciton binding energy $\left(E_{b}\right)$ to the LO phonon energy ( $h \omega_{\mathrm{LO}}=31.8 \mathrm{meV}$ in $\mathrm{ZnSe}$ ), and on the actual strength of the exciton-phonon coupling. The latter parameter can be approximately estimated from the temperature-dependent absorption linewidth of the ground level $\mathrm{HH}$ exciton. In order to get information on this important parameter and, more in general, on the excitonic eigenstates and binding energy, one has to model the absorption line-shape with a statistical model. The absorption spectra are fitted to Gaussian line-shapes (Cingolani et al., 1995; Pellegrini et al., 1995), with linewidths mainly reflecting the inhomogeneous broadening of the states - i.e., statistical fluctuations in the ternary alloy composition, strain and layer thickness $\left(\Gamma_{\text {inh }}\right)$-and the homogeneous term due to lifetime broadening caused by the excitonphonon interaction. The full width at half maximum (FWHM) of the exciton can be written as:

$$
\Gamma(T)=\Gamma_{\mathrm{inh}}+\frac{\Gamma_{L o}}{\left[\left(\exp \left(\hbar \omega_{\mathrm{LO}} / k_{B} T\right)-1\right)\right]}
$$

The homogeneous term, proportional to the LO phonon population, becomes important at high temperature and is weighted by the electronphonon coupling constant $\Gamma_{\text {Lo }}$. The step-like continuum of the quantum well density of states is simulated by a step function, convoluted with the same inhomogeneous Gaussian broadening used to reproduce the exciton absorption resonance, and with the inclusion of the Coulomb enhancement factor at the band edge (Sommerfeld factor) (see for instance Chemla et al., 1984), which is found to play a minor role in these II-VI materials. The binding energy is obtained from the spectral separation between the exciton line and the continuum edge. This simple criterion is justified by the lack of any feature associated with the $2 s$ state of the exciton in the absorption spectra, due to the unavoidable broadening induced by local inhomogeneities of the ternary alloy well.

An example of the result of this line-shape analysis is shown in Figure 6 for a $\mathrm{Zn}_{0.87} \mathrm{Cd}_{0.13} \mathrm{Se} / \mathrm{ZnSe} \mathrm{MQW}$ with $L_{W}=3 \mathrm{~nm}$. The overall calculated absorption spectrum (solid line) obtained from a best fit to the data (solid circles) reproduces fairly well the experimental spectrum. The individual contributions of the heavy-hole $(\mathrm{HH})$ and light-hole $(\mathrm{LH})$ excitonic features and the $\mathrm{HH}$ continuum are also shown (dashed lines).

The analysis of the temperature-dependent absorption spectra of $\mathrm{ZnCdSe} /$ ZnSe MQWs (Pelekanos et al., 1992a; Cingolani et al., 1995) by means of $\mathrm{Eq}$ (7) (neglecting the contribution of acoustic-phonon scattering [Lee et al., 


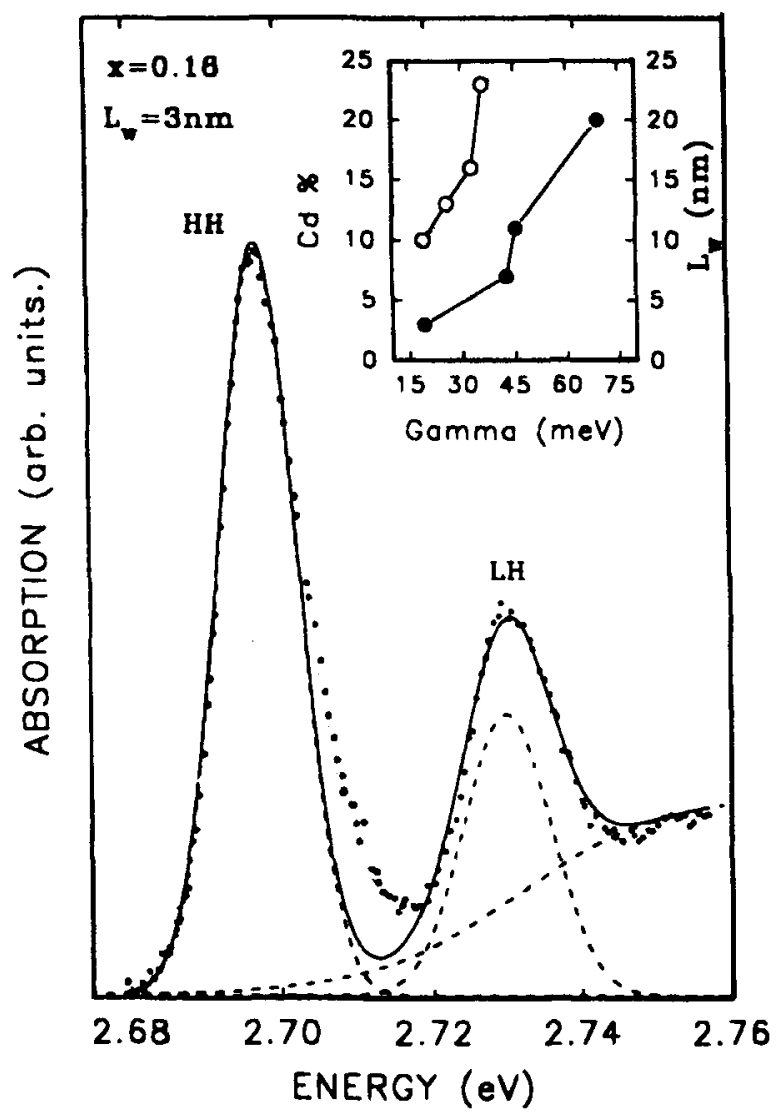

FIG. 6. Line-shape fitting of the absorption spectrum at $10 \mathrm{~K}$ of a $\mathrm{Zn}_{0.84} \mathrm{Cd}_{0.16} \mathrm{Se} / \mathrm{ZnSe}$ multiple quantum well with $L_{w}=3 \mathrm{~nm}$. The overall best fit (continuous line) to the experimental data (solid circles) is shown together with the individual heavy-hole (HH) and light-hole (LH) exciton resonances and HH continuum (dashed lines). Inset: exciton-phonon coupling constant $\left(\Gamma_{\mathrm{LO}}\right)$ versus the well width $L_{w}{ }^{e}$ at $x=0.11$ (solid circles, right-hand scale) and versus $\mathrm{Cd}$ concentration (empty circles, left-hand scale), as obtained from the temperature-dependent exciton linewidth.

1986]) provides the $\Gamma_{\mathrm{Lo}}$ values displayed in the inset of Figure 6. The exciton-phonon coupling in narrow QWs $(3 \mathrm{~nm})$ is found to be smaller than in bulk $\mathrm{ZnSe}$, whereas for wide wells $(20 \mathrm{~nm})$ it is larger than in the bulk, in agreement with the theoretical work of Young et al. (1994). The important implication of this result is that the reduced exciton-phonon coupling in narrow wells favors the exciton stability, leading to a dominant excitonic role in the optical processes of II-VI quantum wells under strong injection 
or high temperatures. We also observe in the inset of Figure 6 that, for a given well width $(3 \mathrm{~nm})$, the exciton-phonon coupling appears to increase with increasing $\mathrm{Cd}$ content. Apparently, the increased exciton confinement occurring in deep $\mathrm{Zn}_{1-x} \mathrm{Cd}_{x} \mathrm{Se} / \mathrm{ZnSe} \mathrm{QWs}$ is partly balanced by the increased exciton-phonon coupling occurring in the Cd-rich alloys. This result is in contrast with the expected reduction of the exciton-phonon coupling occurring in CdSe with respect to $\mathrm{ZnSe}$ (Rudin et al., 1990). A totally different situation is found for the $\mathrm{ZnSe} / \mathrm{ZnS}$ system, in which the weak confinement does not affect strongly the phonon coupling. Strong exciton-phonon interaction is indeed observed in the optical spectra, as described at the end of this subsection.

In wide wells with large $\mathrm{Cd}$ content $(x>0.2)$ the broadening becomes comparable to the subband splitting, and the interband continuum can hardly be resolved in the experimental spectra. For example, in the investigated composition range $(0.1<x<0.3)$ a $\pm 1 \%$ fluctuation in the $\mathrm{Cd}$ content of the alloy results in a fluctuation of $\pm 11 \mathrm{meV}$ in the energy gap (Tamargo et al., 1991). The corresponding unintentional modulation of the quantum well depth would cause per se a fluctuation of $1-3 \mathrm{meV}$ in the quantization energies of the carriers in the well.

The experimental exciton binding energies are summarized in Figure 7 for the different MQWs (solid circles and triangles for $x=0.11$ and $x=0.23$ QWs, respectively), together with the results of the variational calculations described in Section II (solid lines). For shallow MQWs, the calculated exciton binding energy varies from the bulk value (about $19 \mathrm{meV}$ for $L_{W}=20 \mathrm{~nm}$ ) up to $32 \mathrm{meV}$ for $L_{W}=3 \mathrm{~nm}$. For even lower well widths the calculated exciton binding energy decreases, due to the increased penetration of the exciton wavefunction in the barriers. Deeper quantum wells $(x=0.23)$ exhibit a similar behavior, but the maximum exciton binding energy is increased to about $37 \mathrm{meV}$ for $L_{W}=2 \mathrm{~nm}$. Similar values are reported by other authors (Pelekanos et al., 1992b; Liaci et al., 1995; Pellegrini et al., 1995). Some discrepancy is found in the wide well region $\left(L_{W}=20 \mathrm{~nm}\right)$, and is likely to reflect the limited accuracy of the trial wavefunction Eq. (4) when the 3D exciton limit is approached (Miller et al., 1985; Bastard, 1991; Liaci et al., 1995).

These results clearly show that exciton confinement in $\mathrm{Zn}_{1-x} \mathrm{Cd}_{x} \mathrm{Se} / \mathrm{ZnSe}$ MQWs can be tuned independently by varying the well composition or thickness. For a given well width of $3 \mathrm{~nm}$, the increase of the well depth (i.e., the increase of the Cd content from typically $x=0.1$ to $x=0.25$ ) results in a $15 \%$ enhancement of the exciton binding energy, reflecting the enhanced localization of the carrier wavefunctions in the well. This is shown more clearly in Figure 8, where we plot calculated (solid and dashed lines) and experimental (solid circles) ground state exciton binding energies as a 


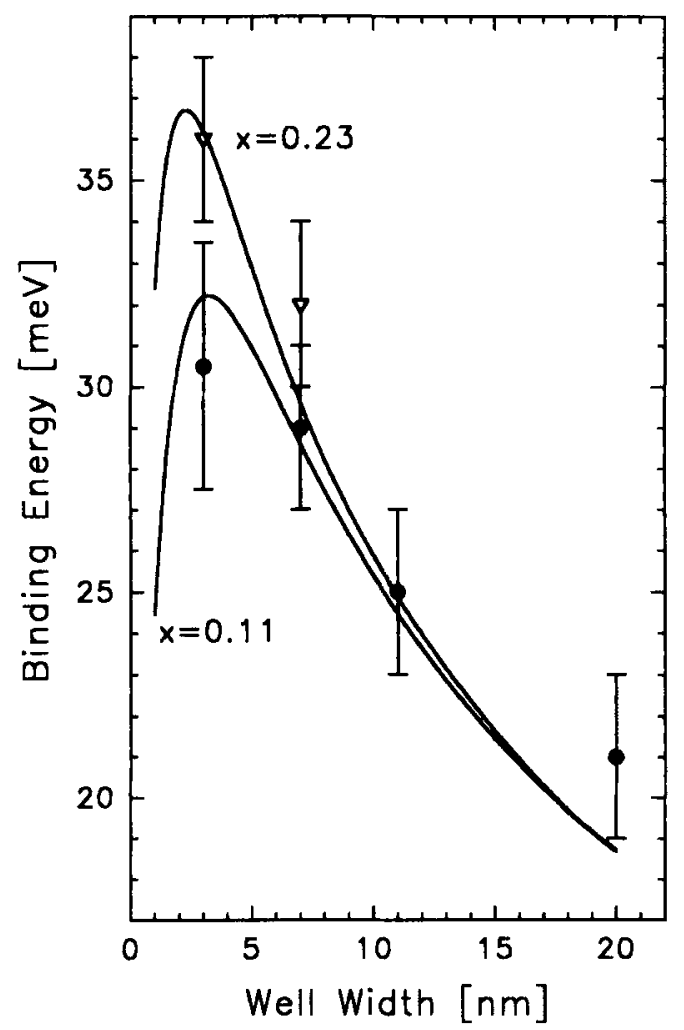

FiG. 7. Experimental exciton binding energy of $\mathrm{Zn}_{(1-x)} \mathrm{Cd}_{x} \mathrm{Se} / \mathrm{ZnSe}$ quantum wells with $x=0.11$ (circles) and $x=0.23$ (triangles) as a function of the well width. The curves are the results of variational calculations taking a linear interpolation of the dielectric constants $[\varepsilon=8.8(1-x)+9.3 x]$.

function of $\mathrm{Cd}$ content. The exciton binding energy is clearly seen to vary from just below to above the LO phonon energy in the well composition range examined.

The data of Figures 7 and 8 suggest that maximum exciton stability $\left(E_{b}>\hbar \omega_{\text {Lo }}\right)$ cannot be achieved in shallow quantum wells $(x \simeq 0.1)$. Conversely, a strongly stabilized exciton exists in the deeper quantum wells $(x \geqslant 0.2)$, at least for sufficiently narrow well widths, as shown, for example, by the presence of a clear excitonic resonance in the room temperature absorption spectrum of Figure 5a. Under the condition $E_{b}>\hbar \omega_{\mathrm{LO}}$, excitons are thus expected to play an important role in real devices operating at high temperatures and strong injection rates, namely lasers and modulators. 


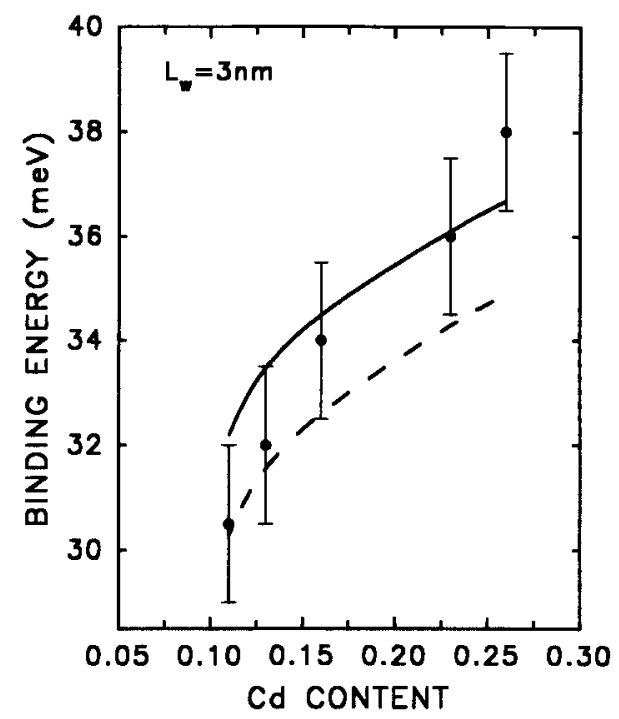

FIG. 8. Compositional dependence of the exciton binding energy of $\mathrm{Zn}_{(1-x)} \mathrm{Cd}_{x} \mathrm{Se} / \mathrm{ZnSe}$ with well width $L_{w}=3 \mathrm{~nm}$. The curves represent the results of variational calculations taking a linear interpolation of the dielectric constants (continuous) or the CdSe bulk value $\varepsilon=9.3$ (dashed).

We have now all of the information required to evaluate the interband transition energies of $\mathrm{ZnCdSe} / \mathrm{ZnSe} \mathrm{MQWs}$. In Figure 9 we compare transition energies calculated in the envelope function approximation including strain (see Section II) with the experimental values obtained by adding the binding energy data in Figure 7 to the experimental energy position of HH and LH resonances (solid and open circles in Figure 9, respectively). The calculated $\mathrm{HH}$ interband transitions with quantum number $n=1,2,3$ (solid lines) and LH (dashed lines) match within $10 \mathrm{meV}$ the experimental values (solid and open circles), for both $\mathrm{Zn}_{0.89} \mathrm{Cd}_{0.11} \mathrm{Se} / \mathrm{ZnSe}$ (topmost section) and $\mathrm{Zn}_{0.77} \mathrm{Cd}_{0.23} \mathrm{Se} / \mathrm{ZnSe} \mathrm{MQWs}$. Such a difference is comparable to the experimental uncertainty expected for fluctuations of the order of $\pm 1 \%$ in the well composition, and/or monolayer fluctuations in the width of narrow wells. Similar agreement is also found in Figure 10, where we plot the $\mathrm{Cd}$ dependence of the ground level $\mathrm{HH}$ and $\mathrm{LH}$ excitonic transition energies for a series of MQWs with $L_{W}=3 \mathrm{~nm}$ and well composition $x$ spanning the 0.10-0.26 range. We should mention that most of the band parameters of $\mathrm{ZnSe}$, zincblende $\mathrm{CdSe}$, and related ternary alloy are not well known (Landolt and Bornstein, 1982). The remarkable overall agreement between calculated and measured transition energies supports the 


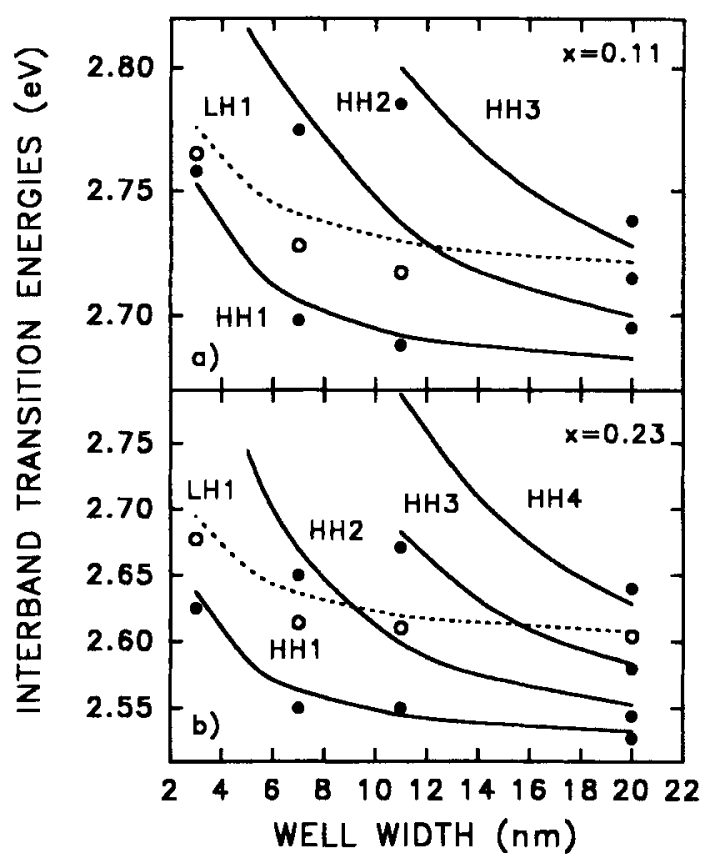

FIG. 9. Interband heavy-hole (HH, solid circles) and light-hole (LH, empty circles) transition energies of $\mathrm{Zn}_{(1-x)} \mathrm{Cd}_{x} \mathrm{Se} / \mathrm{ZnSe}$ quantum wells with $x=0.11$ and $x=0.23$ at $10 \mathrm{~K}$. The curves represent the results of envelope function calculations in the effective mass approximation, including strain. The continuous curves denote $\mathrm{HH}$ transitions with quantum number $n=1,2,3 \ldots$, whereas the dashed curves represent the $n=1 \mathrm{LH}$ transition. The experimental values are obtained by adding the exciton binding energies of Figure 7 to the experimental energy position of the $\mathrm{HH}$ and $\mathrm{LH}$ excitonic resonances measured in absorption.

choice of band parameters (Table I) and exciton binding energies used here. Many theoretical and experimental studies of excitons in $\mathrm{ZnCdSe} / \mathrm{ZnSe}$ MQWs have used quite different values for the conduction and valence band effective masses, deformation potentials, and band offsets (Ding et al., 1990b; Ding et al., 1992; Pelekanos et al., 1992b; Lozykowsky and Shastri, 1992; Alonso et al., 1992; Ding et al., 1993; Chung et al., 1993; Ren et al., 1994; Young et al., 1994; Cingolani et al., 1995; Pellegrini et al., 1995; Liaci et al., 1995) leading to slightly different results. The major problem in the eigenstate calculations is the interplay between the band offset expected for the bulk, unstrained materials and the effect of tetragonal strain field on the band extrema in determining the actual total band discontinuities. Recent data reported in the literature about $\mathrm{ZnCdSe} / \mathrm{ZnSe}$ indicate that the conduction band offset is much larger than the valence band offset, and 


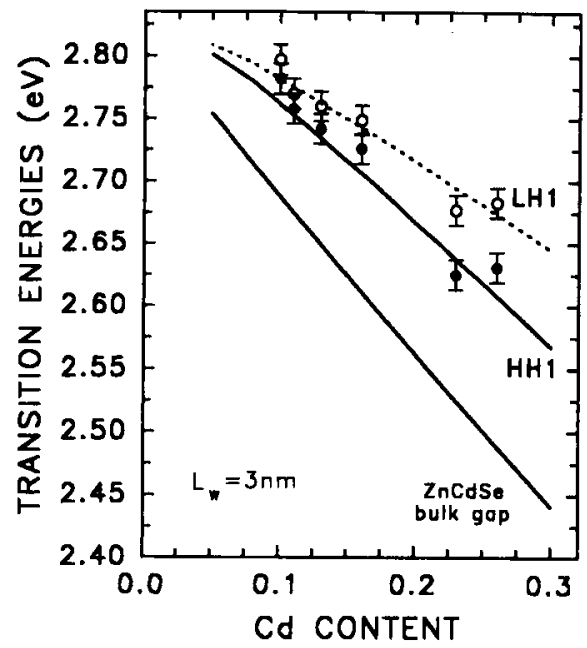

FIG. 10. Compositional dependence of the interband transition energies of $\mathrm{Zn}_{(1-x)} \mathrm{Cd}_{x} \mathrm{Se}$ / $\mathrm{ZnSe}$ quantum wells with $L_{w}=3 \mathrm{~nm}$ at $10 \mathrm{~K}$. The solid and dashed lines correspond to the calculated $\mathrm{HH}$ and LH transition energy, respectively. A constant light-hole exciton binding energy of $20 \mathrm{meV}$ has been assumed in the calculations. The energy gap of the unstrained bulk ternary alloy $\mathrm{Zn}_{(1-x)} \mathrm{Cd}_{x}$ Se is also plotted for comparison (bottommost solid line).

ranges between 0.65 and 0.85 of the band gap difference. The offset value is usually obtained by comparing the energy of the higher index transitions measured by absorption (Ding et al., 1990a; Cingolani et al., 1995; Pellegrini et al., 1995) or reflectivity (Alonso et al., 1992; Liaci et al., 1995) with envelope function calculations. The spread of these determinations thus depends on the choice of the effective masses, and structural parameters for the strain evaluation, and, in most cases, on the poor assessment of the well width and composition. On the other hand, the theoretical prediction of the offset (Chung et al., 1993; Ren et al., 1994) suffers the typical indetermination of band structure calculations which is of the order of several tens of $\mathrm{meV}$.

Nevertheless, the different choice of band offset ratio and/or $\alpha_{c}$ and $\alpha_{v}$ (see Section II) only slightly affects the agreement between calculated and experimental transition energies for the higher index excitonic eigenstates of technologically interesting $\mathrm{Zn}_{1-x} \mathrm{Cd}_{x}$ Se quantum wells (with $x<0.3$ ), due to large effective masses and limited potential depth. For the specific case of Figure 9, we performed several calculations for different values of the conduction band offset (in the 0.7--0.9 $\Delta E_{g}$ range) and with $\alpha_{c}=0$, (ii) $\alpha_{c}=2 / 3 \alpha$, and (iii) $\alpha_{c}=\alpha$. The combination of parameters that was found to better reproduce the experimental results was a conduction band offset equal to about $0.8 \Delta E_{g}$ and $\alpha_{c}=2 / 3 \alpha$. 
Another important issue is the evaluation of the binding energy of the light-hole exciton. In fact, in these heterostructures the combination of strain and the relatively low valence band offset leads to a vanishing confinement potential for the LH states, largely independent of well width and composition. In particular, the depth of the LH potential well varies between $3 \mathrm{meV}$ at $x=0.1$ and $15 \mathrm{meV}$ at $x=0.3$, causing the light-hole states to be weakly confined or resonant with the continuum. This explains why the LH excitonic features readily disappear from the absorption spectra with increasing temperature. In the calculations of Figure 9 we have used the model of Galbraith (1992) for quantum wells of vanishing band offset, to evaluate a binding energy of about $20 \mathrm{meV}$ for the $\mathrm{LH}$ exciton in $\mathrm{ZnCdSe} / \mathrm{ZnSe}$ MQWs, irrespective of the well width and composition. More accurate results for the light-hole exciton binding energy can be found in the work of Liaci et al. (1995).

A totally different scenario is found for the companion $\mathrm{ZnSe} / \mathrm{ZnS}$ material system. In this case the relevant property is that the conduction band offset is about $10 \%$ of the total band gap discontinuity. This value is well documented either theoretically (Quiroga et al., 1990; Bertho and Jouanin, 1993) or experimentally (Mohammed et al., 1987; Shahzad et al., 1988; Shen et al., 1992; Gil et al., 1994; Cingolani et al., 1994b) for $\mathrm{ZnSe} / \mathrm{ZnSSe}$ heterostructures with different sulfur content. The conduction band offset is reduced to a few $\mathrm{meV}$ by the strain, resulting in an almost flat conduction band in heterostructures consisting of ternary $\mathrm{ZnS}_{x} \mathrm{Se}_{1-x}$ barriers (with $x<0.3$ ). The major consequences of this characteristic are (i) the formation of almost delocalized conduction-band states, (ii) a bulk-like exciton binding energy, and (iii) eventually the occurrence of type II band alignment in the presence of large strain or moderate external hydrostatic pressures (Yamada et al., 1991; Lomascolo et al., 1994; Gorczyca and Christensen, 1993).

The excitonic properties of these materials are strongly affected by the weak electronic confinement, and are primarily determined by the quantization of the valence band states. Other materials in which a similar situation occurs are the $\mathrm{CdTe} / \mathrm{ZnCdTe}$ or $\mathrm{ZnSe} / \mathrm{ZnMgSe}$ structures, having little or no valence band offset and strong electron confinement. Several authors have carried out calculations to model these situations. Wu and Nurmikko (1988) have used a modified band edge potential to confine the unconfined particle by the Coulomb force. Galbraith (1992) used a variational technique assuming an anisotropic exciton in which the electron is bound to the hole represented by a delta function. This technique, though giving a weak enhancement of the exciton binding energy (in agreement with the experiments), results in an unphysical independence of the binding energy on the well width. The use of the simple model outlined in Section II provides a 
more correct description of the exciton in these materials, in which the weak electron confinement is treated by using explicitly the heavy-hole and electron wavefunctions. The precise determination of the conduction band offset is crucial for the determination of the eigenstates and exciton binding energy. This has been achieved by photoluminescence (PL) experiments under high hydrostatic pressure. The application of hydrostatic pressure is expected to cause significant relative changes of conduction band offset in these superlattices. This is due to the difference in the band gap pressure coefficients of $\mathrm{ZnSe}(72 \mathrm{meV} / \mathrm{GPa})(\mathrm{Hwang}$ et al., 1994) and $\mathrm{ZnS}$ $(64 \mathrm{meV} / \mathrm{GPa})$. At some critical pressure the $\Gamma$ point of $\mathrm{ZnSe}$ is expected to shift at higher energy than that of the ZnSSe alloy, so that electrons are confined in the barrier and holes remain in the well, resulting in a pressure-induced type I-type II transition. Evidence for this transition has been reported for $\mathrm{ZnSe} / \mathrm{ZnS}$ (Yamada et al., 1991) and for $\mathrm{ZnSe} / \mathrm{ZnSSe}$ superlattices (Lomascolo et al., 1994). Above the critical pressure the transition was monitored through (i) the strong decrease of the PL intensity, (ii) the appearance of different emission bands in the PL spectra, and (iii) by the change of the phonon sideband energies, reflecting the change from $\mathrm{ZnSe}$ LO phonons to ZnSSe LO phonons. These results are summarized in Figure 11 for a $2.8 \mathrm{~nm} / 2.8 \mathrm{~nm} \mathrm{ZnSe} / \mathrm{ZnS}_{0.18} \mathrm{Se}_{0.82}$ superlattice measured at low temperature. The type I-type II transition occurs around $4.5 \mathrm{GPa}$, when the integrated emission intensity drops abruptly. At the critical pressure value the $\Gamma$ points of the well and the barrier are aligned, corresponding to a conduction and offset of $6-7 \mathrm{meV}$. At the same pressure a change in the splitting of the PL phonon sidebands is observed, consistent with the change from the $\mathrm{ZnSe} \mathrm{LO}$ phonon to the $\mathrm{ZnS}_{0.18} \mathrm{Se}_{0.82} \mathrm{LO}$ phonon assisted recombination $\left(\hbar \omega_{\mathrm{LO}}^{\mathrm{ZnSe}}=31.8 \mathrm{meV}\right.$ and $\hbar \omega_{\mathrm{LO}}^{\mathrm{ZnSSe}}=29.3 \mathrm{meV}$, respectively).

The precise evaluation of the conduction band offset allows us to evaluate the small electron confinement energy and the exciton binding energy of the $\mathrm{ZnSe} / \mathrm{ZnS}_{0.18} \mathrm{Se}_{0.82}$ superlattices. These values are compared to the experimental absorption and photoluminescence excitation data, in a way similar to the $\mathrm{ZnCdSe} / \mathrm{ZnSe}$ case. In Figure 12 we show the transmission spectrum of a $\mathrm{ZnSe} / \mathrm{ZnSSe} \mathrm{SL}$, consisting of 80 periods of $3.7 \mathrm{~nm} \mathrm{ZnSe} / 3.7 \mathrm{~nm}$ $\mathrm{ZnS}_{0.8} \mathrm{Se}_{0.82}$. The line-shape analysis reveals a distinct edge of the continuum, from which a binding energy of about $22 \mathrm{meV}$ is estimated. The systematic analysis of symmetric superlattices with well widths comprised in the range $2 \mathrm{~nm}<L_{x}<15 \mathrm{~nm}$, reveals the expected weak dependence of the exciton binding energy on the well width (Fig. 13). Unlike the case of the $\mathrm{ZnCdSe} \mathrm{MQWs}$, the exciton binding energy increases only by about $25 \%$ in the narrowest quantum wells, clearly showing the important role of the electron delocalization in heterostructures with negligible conduction band offset. Similar absorption experiments have been performed in $\mathrm{ZnS} / \mathrm{ZnSe}$ 

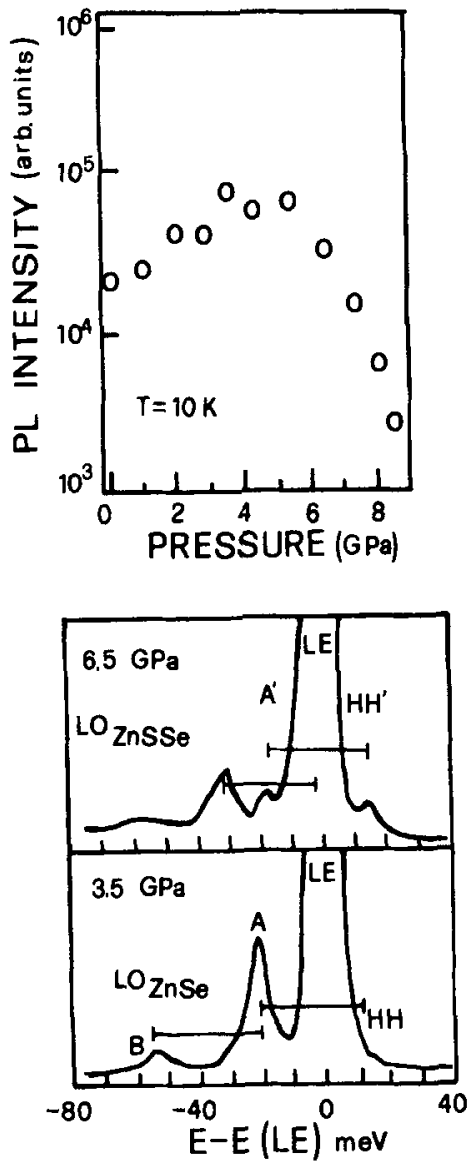

\section{$\mathrm{ZnSe} / \mathrm{ZnSSe}$}

$L_{W}=2.8 \mathrm{~nm}$

$P=O G P a$

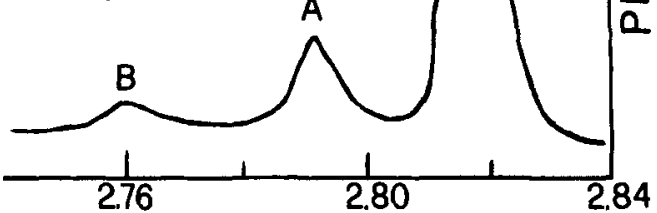

ENERGY (eV)

FIG. 11. Low-temperature continuous-wave luminescence spectrum recorded from a symmetric $\mathrm{ZnS}_{0.18} \mathrm{Se}_{0.82} / \mathrm{ZnSe}$ superlattice of well and barrier width equal to $2.8 \mathrm{~nm}$. LE is the emission of excitons localized at flat islands at the interfaces, A and B are the LO-phonon replicas of the heavy-hole exciton $(\mathrm{HH})$. Top inset: LE emission intensity versus the hydrostatic pressure. The type I-type II transition is monitored through the drop in intensity occurring at 4.5 GPa. Bottom inset: Fine structure of the luminescence spectra recorded just below and above the pressure-induced type I-type II cross-over. Below threshold the phonon-assisted transitions (A,B) involve $\mathrm{ZnSe}$ LO phonons $(31.8 \mathrm{meV})$. Above threshold, the phonon replicas involve ZnSSe LO phonons. 


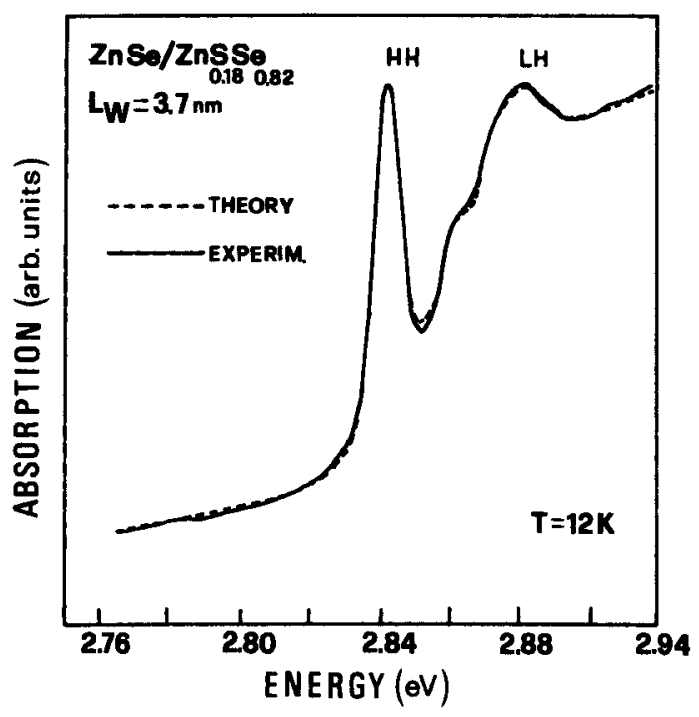

FIG. 12. Experimental and calculated absorption spectrum of a symmetric $\mathrm{ZnS}_{0.18} \mathrm{Se}_{0.82} /$ $\mathrm{ZnSe}$ superlattice (well and barrier width equal to $3.7 \mathrm{~nm}$ ) at $12 \mathrm{~K}$.

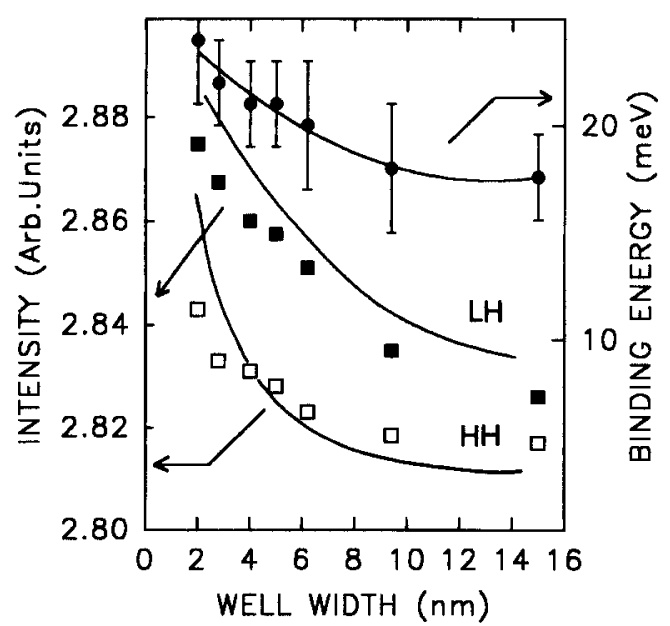

FIG. 13. Well width dependence of the exciton binding energy (circles, right-hand scale) and of the excitonic transition energies (squares, left-hand scale) at $10 \mathrm{~K}$ in symmetric $\mathrm{ZnS}_{0.18} \mathrm{Se}_{0.82} / \mathrm{ZnSe}$ superlattices. The curve interpolating the binding energy symbols is a guide for the eye. The curves reproducing the excitonic energies have been calculated according to the envelope function model, assuming a free-standing superlattice (total thickness about $1 \mu$ ). 
(Shen et al., 1992; Hohnoki et al., 1994) and CdSe/ZnSe heterostructures (Shan et al., 1993; Yang et al., 1993).

The inclusion of the exciton binding energy, of the experimentally estimated conduction band offset, and of the strain corrections in a KrönigPenney model, gives a satisfactory description of the well width dependence of the HH and LH energies in Figure 13 (Cingolani, 1994b). The total exciton energy increases by less than $60 \mathrm{meV}$ in the investigated well width range, because the confinement is dominated by the hole quantization. The discrepancy between experiment and theory is affected by the lack of information on the effective masses and deformation potentials. Furthermore, the assumption of totally free-standing superlattices used in the strain calculations is ideal (the superlattices used in this study had a total thickness of about $1 \mu \mathrm{m}$ ), and might be not totally correct in real samples.

Before concluding this section we recall that no reduction of the electron-phonon coupling is expected in $\mathrm{ZnSe} / \mathrm{ZnSSe}$ superlattices with respect to bulk, due to the weak confinement. This is reflected not only in the weak

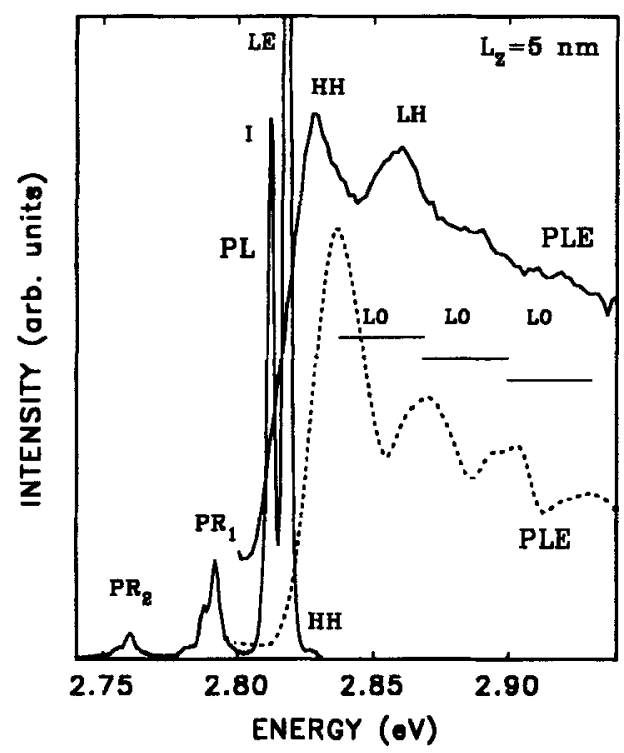

Fig. 14. Photoluminescence (PL) and photoluminescence excitation spectra (PLE) of a $5 \mathrm{~nm} / 5 \mathrm{~nm} \mathrm{ZnS}{ }_{0.18} \mathrm{Se}_{0.82} / \mathrm{ZnSe}$ superlattice at $10 \mathrm{~K}$. The PL spectrum shows sharp localizedstate emission (LE,I) and phonon replicas (PR). The PLE spectrum recorded under low excitation power (dashed curve) exhibits distinct oscillation with periodicity of one LO phonon, characteristic of hot-exciton generation. The PLE measured under higher excitation power recovers the density of states line-shape with distinct heavy-hole (HH) and light-hole (LH) excitonic resonances. 
enhancement of the exciton binding energy, but also in the appearance of strong hot-exciton features in the photoluminescence excitation (PLE) spectra. This situation is depicted in Figure 14, where we compare the PLE spectra of a $5 \mathrm{~nm} / 5 \mathrm{~nm} \mathrm{ZnSe} / \mathrm{ZnS}_{0.18} \mathrm{Se}_{0.82}$ superlattice recorded under different excitation intensities. At very low power (of the order of few $\mu \mathrm{W} \mathrm{cm}{ }^{-2}$ ), the formation of hot excitons can be observed in the PLE spectra, through the characteristic oscillatory behavior of period equal to the $\mathrm{ZnSe}$ LO-phonon energy (Cingolani et al., 1994b). This is due to the enhancement of the excitonic absorption in resonance with the phononassisted transition in the continuum. With increasing the excitation intensity (of the order of $1 \mathrm{~mW} \mathrm{~cm} \mathrm{~m}^{-2}$ ), intercarrier scattering becomes a dominant relaxation mechanism, and the usual density of states line-shape is recovered. In this case the PLE spectrum reproduces the usual absorption shape with clear excitonic resonances (continuous curve in Fig. 14).

\section{Excitons at the Dimensionality Cross-over}

Excitons in a quantum well are treated assuming that the lateral barriers influence primarily the wavefunctions of the individual carriers rather than the exciton wavefunction itself, resulting in the so-called strong confinement regime. Under this condition one can reasonably assume that the eigenvalue of the Coulomb interaction (i.e., the exciton binding energy) is smaller or at most comparable to the quantization energy gained by the carriers. In III-V materials this condition is easily fulfilled, due to the large extent of the exciton envelope function (typically $a_{0}>L_{w}$, where $a_{0}$ is the exciton Bohr radius and $L_{w}$ is the well width), and to the light carrier masses (Cingolani and Ploog, 1991). On the contrary, in ZnSe-based quantum wells the exciton Bohr radius is rather small $\left(a_{0} \leqslant 4 \mathrm{~nm}\right)$ whereas the effective masses of carriers are quite large. The condition $a_{0} \leqslant L_{w}$ is easily verified for most common well width values (Fig. 2). Such condition is referred to as the intermediate confinement regime, in which the lateral boundaries influence the exciton envelope function as a whole, rather than the individual carrier wavefunctions, resulting in the so-called quantization of the center of mass (CM) motion of the exciton. This situation is at the crossover between the three-dimensional and the two-dimensional behavior for the excitonic wavefunction.

From the theoretical point of view, we assume that the exciton has a center of mass motion and moves in real space due to its kinetic energy. Coupled with the radiation field it forms an exciton polariton (Hopfield and Thomas, 1963), whose spatial dispersion affects the optical spectra of absorption, photoluminescence, and reflectivity. The fundamental equation 
of the exciton-photon interaction, which gives the polariton dispersion curves $E(k)$, is obtained by equating the dielectric function (assuming Lorentzian excitonic resonances) and the photon dispersion law:

$$
\varepsilon_{\infty}+\frac{4 \pi \alpha_{o} E_{o}^{2}}{E_{o}^{2}-E^{2}+\beta k^{2}+i \gamma E}=\frac{\hbar^{2} c^{2}}{E^{2}} k^{2}
$$

where $\varepsilon_{\infty}$ is the dielectric constant, $4 \pi \alpha_{o}$ is the oscillator strength, $E_{o}$ is the free exciton energy, $\beta=\hbar^{2} E_{o} / M_{\text {exc }}$ with $M_{\text {exc }}$ the effective exciton mass, $\gamma$ is the exciton broadening, $k$ is its wave vector. The momentum of the $\mathrm{CM}$ along the growth direction $\left(k_{z}\right)$ is quantized because the polariton wave is strongly reflected at the well-barrier interfaces. Standing waves then exist for $L_{w}=n \lambda / 2$, where $n$ is a nonzero integer and $\lambda$ is the polariton wavelength. Consequently, the continuous polariton curve $E(k)$ transforms into a series of discrete points at $k_{n}=n\left(\frac{\pi}{L_{w}}\right)$ with $n=1,2,3 \ldots$. This is true for well width values down to about two times the exciton Bohr radius $a_{0}$.

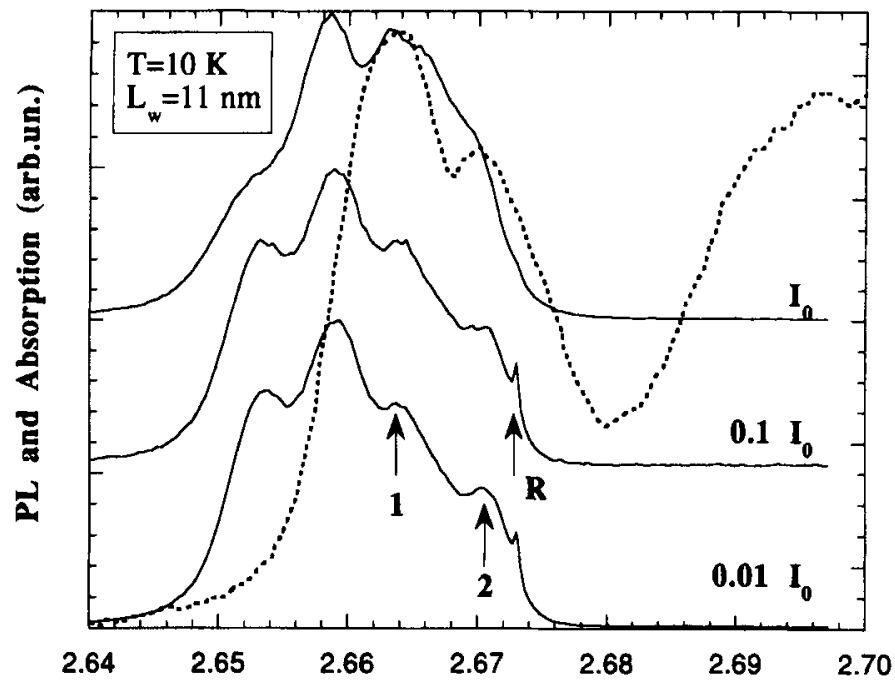

Energy (eV)

Fig. 15. Photoluminescence spectra at different excitation intensities (continuous lines) and absorption spectrum (dashed line) of a $\mathrm{Zn}_{0.89} \mathrm{Cd}_{0.11} \mathrm{Se} / \mathrm{ZnSe} \mathrm{MQWs}$ with $L_{w}=11 \mathrm{~nm}$, recorded at $10 \mathrm{~K} . I_{0}=16 \mathrm{~W} \mathrm{~cm}^{-2}$. The arrows indicate the center of mass states. The peak labeled $\mathbf{R}$ is the Raman line of the exciting laser. 
Under this condition, the energy dispersion of the exciton-polariton becomes

$$
E_{n}^{\mathrm{exc}}=E_{o}+\frac{\hbar^{2}}{2 M_{\mathrm{exc}}}\left(\frac{\pi}{L_{w}}\right)^{2} n^{2}
$$

In Figure 15 we show the PL and absorption spectra of $\mathrm{Zn}_{0.89} \mathrm{Cd}_{0.11} \mathrm{Se} /$ $\mathrm{ZnSe}$ MQWs of well and barrier width $11 \mathrm{~nm}$ and $20 \mathrm{~nm}$, respectively. In the PL spectrum we can distinguish up to four peaks, the lowest energy doublet being extrinsic as deduced from the saturated intensity independence. The comparison between luminescence and absorption spectra confirms the excitonic nature of the other features, which extend over about $10 \mathrm{meV}$ on the high-energy tail of the excitonic resonance. This energy range is smaller than the LO phonon energy in the ternary alloy (about $30 \mathrm{meV}$ ). This prevents fast relaxation of the excited states into the lowest level, resulting in the simultaneous recombination of the excited $\mathrm{CM}$ states.

In Figure 16 we compare the absorption spectra of samples with different well widths $7 \mathrm{~nm}<L_{w}<20 \mathrm{~nm}$ (Greco et al., 1996). This thickness range

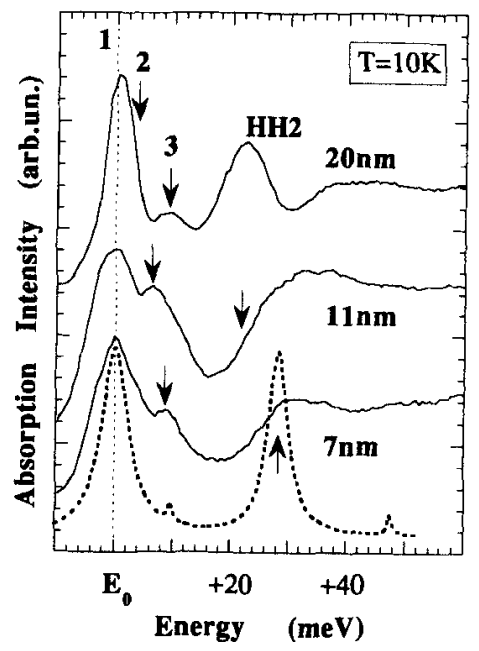

FIG. 16. Comparison of the absorption spectra of $\mathrm{Zn}_{0.89} \mathrm{Cd}_{0.11} \mathrm{Se} / \mathrm{ZnSe} \mathrm{MQWs}$ with different well widths (continuous lines) at $10 \mathrm{~K}$. The arrows indicate center of mass states. The dashed line is the absorption spectrum calculated according to the "thin-slab" variational model discussed in the text (for the $7 \mathrm{~nm}$ MQWs sample). The broadening and the continuum of the quantum wells have been neglected. HH2 labels the $n=2$ exciton resonance of the $20-\mathrm{nm}$ MQWs structure. 

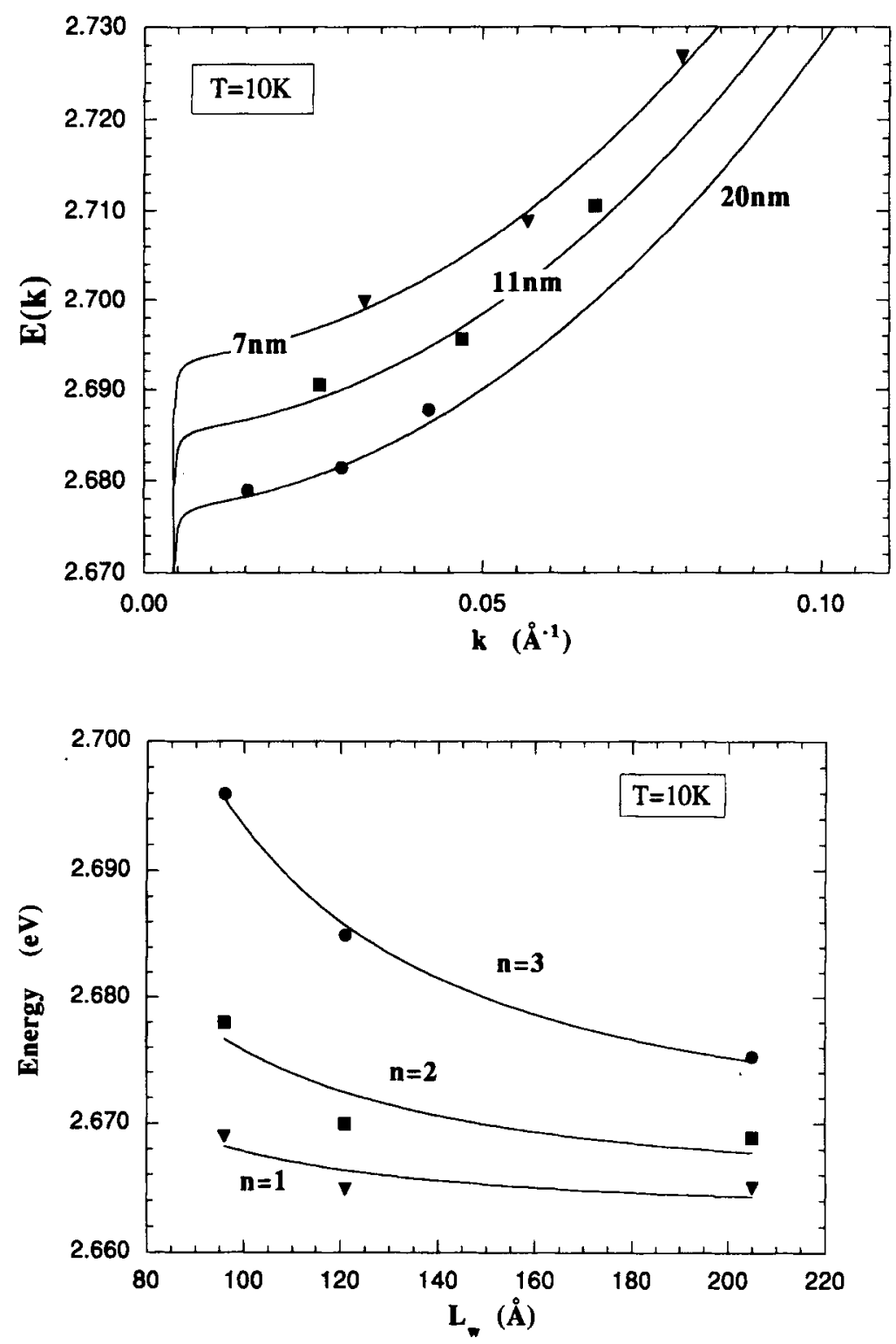

FIG. 17. (a) Energy-momentum dispersion curve of the exciton-polariton calculated by means of the variational model [Eq (10)] and quantized center of mass states (symbols) obtained from the $10 \mathrm{~K}$ absorption spectra of different $\mathrm{ZnCdSe} / \mathrm{ZnSe} \mathrm{MQWs}$. (b) Well width dependence of the center of mass eigenstates (symbols) at $10 \mathrm{~K}$ compared with the energy calculated according to Eq. (9) by using the variational $L_{e q}$. The $n=2$ and $n=3$ curves have been suitably scaled to compare with the data. 
corresponds to the condition $2.5 a_{o}<L_{w}<5 a_{o}$, i.e., to the cross over between two- and three-dimensional crystal. As expected from the $L_{w}$ dependence of the quantization condition (Eq. 9) we observe an increasing splitting of the CM states with decreasing the well thickness. The bulk-like dispersion [Eq (9)] does not reproduce the experimental data unless an unphysical mass enhancement is assumed for the narrower wells. In this case the true exciton-polarition dispersion has to be taken into account. This can be done by using the variational wavefunctions of D'Andrea and Del Sole (1992), valid in the thin-film regime. In this model the exciton is confined in an infinitely deep quantum well of equivalent width $L_{\mathrm{cq}}=L_{w}+2 / P$, where $1 / P$ represents the penetration of the exciton wavefunction in the barrier. $1 / P$ and the exciton Bohr radius are varied in order to minimize the energy of the $\mathrm{CM}$ states. The $\mathrm{CM}$ eigenstates and the dispersion curve can be obtained after the solution of the transcendental equations (D'Andrea and Del Sole, 1990):

$$
\begin{aligned}
k_{n} \tan \left(k_{n} L_{\mathrm{eq}} / 2\right)+P \tanh \left(P L_{\mathrm{eq}} / 2\right) & =0 \\
\frac{\tan \left(k_{n} L_{\mathrm{eq}} / 2\right)}{k_{n}}-\frac{\tanh \left(p L_{\mathrm{eq}} / 2\right)}{P} & =0
\end{aligned}
$$

for even and odd states. Using the variational wavefunctions of D'Andrea and Del Sole (1990) and Eq. (10), we compute the normal incidence optical response of the structures to fit the experimental absorption spectra. The results of the calculations are shown by the dashed line in Figure 16. As we are interested only in the energy-momentum values of the CM states, the actual inhomogeneous broadening and the absorption continuum of the quantum well are neglected in the calculations.

The energy-momentum dispersion for the investigated well widths is plotted in Figure 17a together with the measured CM states. The $K_{n}$ values are obtained after the variational calculation of the absorption spectra. The polariton curve flattens with decreasing well widths. The well width dependence of the CM states is plotted in Figure 17b (Greco et al., 1996). The experimental data are well reproduced by the theory, provided the geometrical well width $L_{w}$ is replaced by the variational $L_{\text {eq }}$ in Eq (9). These data demonstrate that the optical response of $\mathrm{ZnCdSe} / \mathrm{ZnSe} \mathrm{MQWs}$ around the cross-over between two- and three-dimensional crystal is dominated by the quantization of the center of mass motion of the exciton-polariton.

\section{Phototransport Processes}

In this section we discuss the phototransport processes in II-VI quantum wells. These effects are very important for the realization of optoelectronic 
devices, like lasers and nonlinear optical modulators, exploiting the enhanced excitonic properties of II-VI heterostructures. The control of doping for the fabrication of $p-i-n$ heterostructures, and the deposition of highquality ohmic contacts are presently a matter of intense investigation, and presumably much work has still to be done before reaching the technological standard of the III-V materials. These technological limitations prevent the precise determination of the built-in electric field of real devices. On this basis, the present achievements in the field of phototransport have to be considered as very preliminary. Different technological approaches have been pursued to realize II-VI quantum well diodes. In particular either Schottky-barrier diodes or $p-i-n$ heterostructures have been fabricated, with $\mathrm{ZnCdSe} / \mathrm{ZnSe}$ and $\mathrm{ZnSe} / \mathrm{ZnSSe}$ quantum wells. The effect of tunneling and thermoionic emission at the metal/p-ZnSe interface on the current voltage characteristics of $\mathrm{ZnSe} / \mathrm{ZnSSe}$ diodes has been investigated in detail by Suemune (Suemune, 1993). A comparative study of the optical and electrical properties of $p-i-n$ diodes and Schottky-barrier diodes based on MOCVD grown $\mathrm{ZnSe} / \mathrm{ZnSSe}$ samples has demonstrated a considerable improvement in the current-voltage characteristics of these diodes with respect to corresponding MBE-grown $\mathrm{ZnCdSe/ZnSe} \mathrm{superlattices} \mathrm{(Fujii} \mathrm{et} \mathrm{al.,} \mathrm{1994;}$ Suemune et al., 1994). Furthermore, well-resolved excitonic resonances have been observed in the low-temperature $(77 \mathrm{~K})$ photocurrent spectra of $\mathrm{ZnSe} / \mathrm{ZnSSe}$ superlattices (Suemune and Yamanishi, 1993).

Photocurrent spectroscopy has been successfully investigated in $\mathrm{ZnCdSe} /$ ZnSe quantum wells grown in $p-i-n$ configuration (Wang et al., 1993a; Cingolani et al., 1994c; Cingolani et al., 1994d). All these experiments demonstrate that in samples with proper configuration, the photocurrent spectra closely reproduce the actual absorption spectrum of the heterostructure. In Figure 18 we show the layer sequence of a $p-i-n$ diode embedding $\mathrm{ZnCdSe}$ MQWs in the intrinsic region grown on a $p$-type GaAs substrate (Cingolani et al., 1994c). The band profile clearly evidences the advantage of the present configuration. Electron-hole pairs photogenerated at the surface ( $n$-type layer) are readily separated by the internal electric field. The minority carriers experience two favorable potential discontinuities, allowing efficient collection of holes at the bottom contact. Conversely, the majority carriers are retained in the photogeneration region by the double potential barrier. The narrow gap $p$-type layer, besides avoiding the complex problem of $p$-doping of the II-VI substrate, is thus exploited to enhance the minority carrier collection of the heterostructure, i.e., the strength of the electrical signal induced by the absorption of light.

In Figure 19 we show typical photocurrent (PC) spectra measured at different temperatures. The spectra exhibit sharp excitonic features and the distinct edge of the $\mathrm{ZnSe}$ barrier. At low temperature carrier freeze-out 


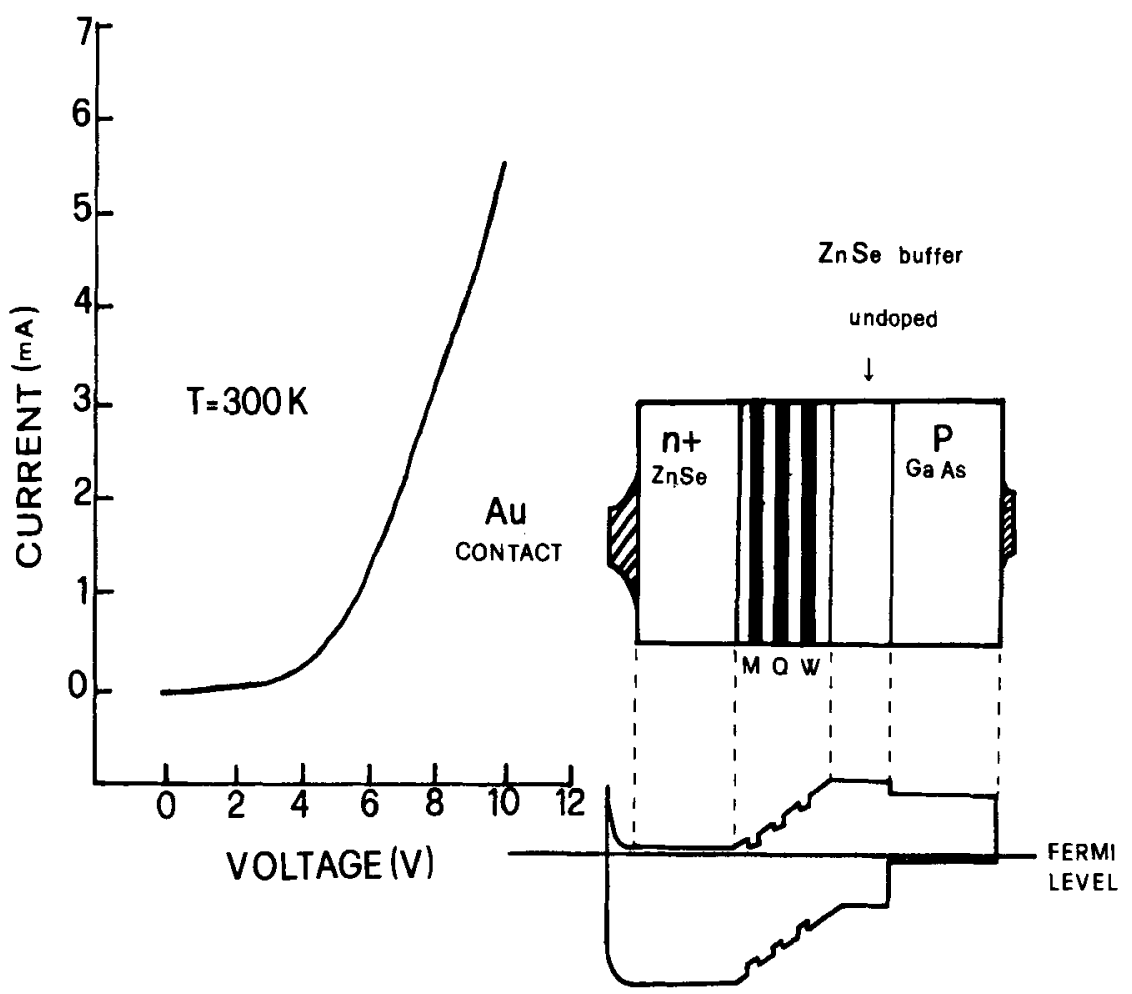

FIG. 18. Right: Schematics of the $p-i-n$ heterostructure embedding $\mathrm{Zn}_{(1-x)} \mathrm{Cd} d_{x} \mathrm{Se} / \mathrm{ZnSe}$ and corresponding band profile in real space, obtained from the self-consistent solution of the Schrödinger and Poisson equation. Left: Room temperature current-voltage characteristic of a structure containing 10 quantum wells with $x=0.10$ and $L_{w}=3 \mathrm{~nm}$.

occurs, and the photocurrent is rather low. With increasing temperature, carriers are thermally activated and the PC line-shape closely reproduces the absorption spectrum (dashed lines in Fig. 19). Following the discussion of Section III.1, the fundamental exciton resonance is seen to disappear at high temperatures in the shallower quantum wells $\left(\hbar \omega_{\mathrm{LO}}>E_{b}\right)$, whereas it remains stable up to room temperature in the deeper QWs.

For a quantitative treatment of the photocurrent process one has to write the continuity equation (Moss et al., 1973)

$$
\frac{d J_{z}}{d z}=e I \alpha e^{-\alpha z}-\frac{\Delta p}{\tau}
$$



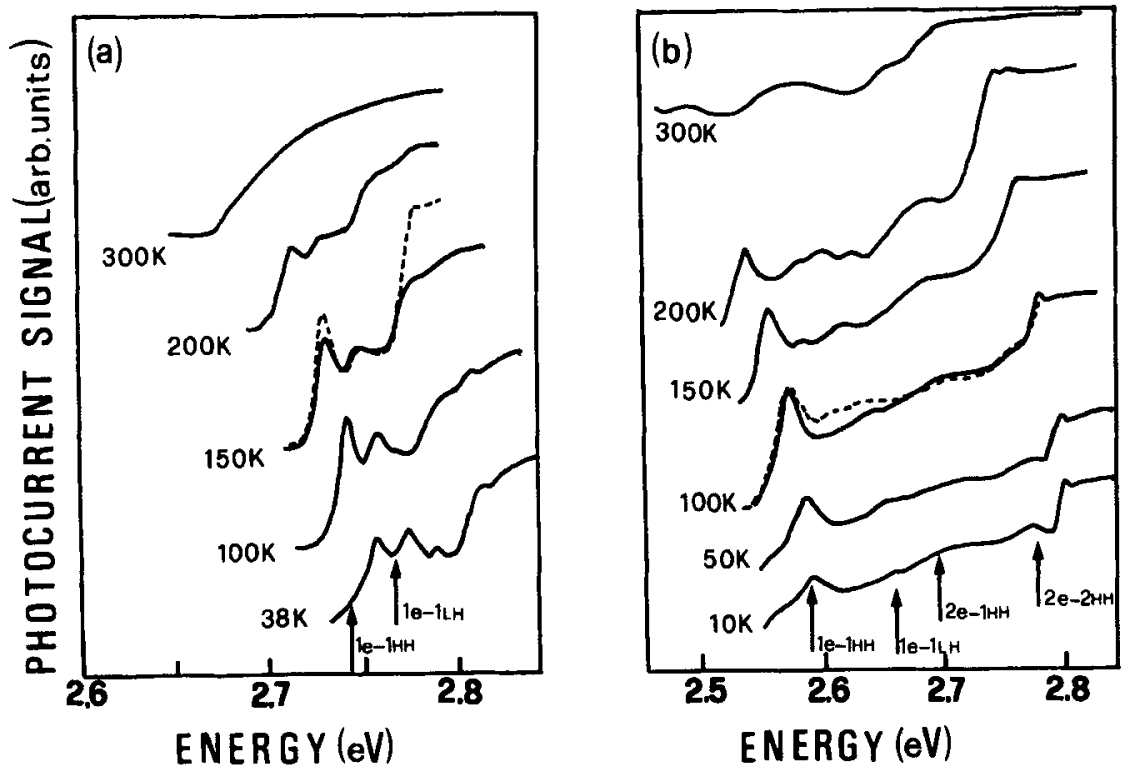

FiG. 19. (a) Temperature-dependent photocurrent spectra recorded from $\mathrm{Zn}_{0.9} \mathrm{Cd}_{0.1} \mathrm{Se} /$ $\mathrm{ZnSe}$ multiple quantum wells embedded in $p-i-n$ heterojunction. The well width is $L_{w}=3 \mathrm{~nm}$. The dashed line represents the measured absorption spectrum. (b) Same as in Figure 19a, but for a $\mathrm{Zn}_{0.75} \mathrm{Cd}_{0.25} \mathrm{Se} / \mathrm{ZnSe} \mathrm{MQWs}$ heterostructure.

and the transport equation

$$
J_{z}=-e D \frac{d}{d z}(\Delta p)+e \mu \Delta p E
$$

Eq (11) consists of two terms: the photogeneration rate depending on the absorption coefficient $\alpha$ ( $I$ is the photon flux) and the nonradiative recombination losses $\tau$ (where $\Delta p$ is the photogenerated excess of minority carriers). The latter includes the surface recombination $e S \Delta p$ (where $S$ is the surface recombination velocity). This loss mechanism is particularly relevant in semiconductor heterostructures with many interfaces. Values as high as $S=10^{5} \mathrm{~cm} \mathrm{sec}^{-1}$ can be reached at the air/semiconductor interface, which reduce by about a factor 1000 or more at the semiconductor/semiconductor interface (Gowar, 1984). The total current density flowing across the heterostructure is given by Eq (12). The major contribution comes from the diffusion term (where $D$ is the diffusion coefficient) and by the drift term, which is negligible in the absence of external polarization ( $\mu$ is the carrier mobility and $E$ is the electric field). 
Eqs (11) and (12) contain the main ingredients for the understanding of the photocurrent spectra of Figure 19. When the energy of the exciting photons exceeds the barrier energy, the light is absorbed directly in the $\mathrm{ZnSe}$ top-layer $(=2.82 \mathrm{eV}$ at $10 \mathrm{~K})$. The minority carriers are thus generated in a thin layer at the surface of the $n$-type $\mathrm{ZnSe}$ contact, from where they have to cross the intrinsic region and the p-type region before reaching the contact. Under these conditions there are two main carrier losses mechanisms: (i) the nonradiative recombination along the carrier path which is longer than the carrier diffusion length, and (ii) the surface recombination at the sample surface. The photogenerated excess of minority carriers (first term of Eq (11)) is partly balanced by the losses. The PC signal is thus not proportional to the absorption as the nonradiative recombination affects the internal carrier collection efficiency. Indeed the $\mathrm{ZnSe}$ region of the PC spectra, though showing a distinct band gap edge with excitonic resonance, does not reproduce quantitatively the strength of the absorption measured by transmission experiments (see the dashed lines in Fig. 19).

When the photon energy is in the transparency region of the $\mathrm{ZnSe}$ barrier, the carriers are generated directly in the quantum wells, with a homogeneous density due to the small total thickness of $\mathrm{ZnCdSe}$. Here they diffuse across the $\mathrm{ZnSe}$ buffer layer toward the GaAs p-contact. Due to the limited distance from the $p$-contact (the total thickness of the MQW region is $L=30 \mathrm{~nm}$ ) and the lack of free surfaces, the collection efficiency is rather large, resulting in a PC signal proportional to the actual absorption of the quantum well. This means that the loss terms in $\mathrm{Eq}$ (11) give a minor contribution to the total photogenerated current. Indeed, in the limit of vanishingly small loss terms in Eq (11), one obtains

$$
J_{z}=e \alpha I \int_{0}^{L} e^{-\alpha z} d z=e I\left(1-e^{-\alpha z}\right) \simeq e I \alpha L
$$

Under this condition, the PC signal is directly proportional to the absorption coefficient. This finding is confirmed by the close similarity of the QW absorption and photocurrent spectra in Figure 19. The important role of the surface recombination is further evidenced when the free surface surrounding the metallic contact on top of the sample is chemically removed by deep chemical etching. Indeed, dramatic improvement in the PC response of the structures is obtained after fabricating mesa-structures of about $800 \mathrm{~nm}$ depth and area coincident with the metallic contact itself, as shown in Figure 20 . The photocurrent signal increases by a factor 10 , even without bias, and the excitonic features are strongly sharpened, especially in the bulk $\mathrm{ZnSe}$ region. In this case the PC spectrum really reproduces the absorption 


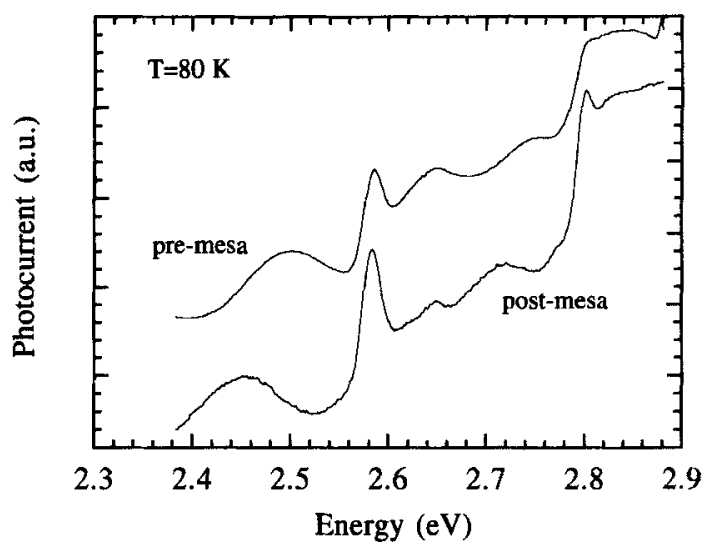

FIG. 20. Photocurrent spectra recorded before and after the fabrication of submillimetric mesas from a $\mathrm{Zn}_{0.9} \mathrm{Cd}_{0.1} \mathrm{Se} / \mathrm{ZnSe}$ multiple quantum well structure in $p-i-n$ configuration.

spectrum. This is a direct consequence of the reduction of the surface recombination velocity in the illuminated region. This suggests that the surface recombination velocity is the dominant carrier loss mechanism reducing the internal quantum efficiency of these II-VI heterostructures.

Finally we analyze the PC spectra under external polarization. For the specific case of Figure 18, no shift or bleaching of the exciton resonance is observed under reverse bias, because of the modest internal field (of the order of $10^{4} \cdot \mathrm{V} \mathrm{cm}^{-1}$ ) due to the large total thickness of the intrinsic area and to the series resistance effects at the nonohmic contacts. However, dramatic changes in the spectral line-shape of the photocurrent are observed for reverse bias around the breakdown field. These are shown in Figure 21 where we can see that the excitonic peaks observed at zero bias progressively transform into sharp dips with increasing bias. For polarization values corresponding to the breakdown value $(-4.5 \mathrm{~V})$ the $\mathrm{PC}$ spectra become flat, then the sign reversal of the PC signal occurs for larger negative voltages. Similar effects have been observed in the PC spectra of III-V heterostructures under similar experimental conditions (Marshall et al., 1989; Leavitt and Bradshaw, 1991; Grahan et al., 1992; Yokuda et al., 1994). At low field the transit time of the carriers across the intrinsic region containing thick barriers can be longer than the carrier lifetime, resulting in a poor carrier collection at the bottom contact. On the contrary, at higher fields or in the presence of thin barriers, the transit time and the lifetime of the carriers can be comparable. These effects manifest themselves with a sudden change of the PC line-shape, as the one observed in Figure 21. It is worth noting that the sign reversal of the PC resonances disappears 


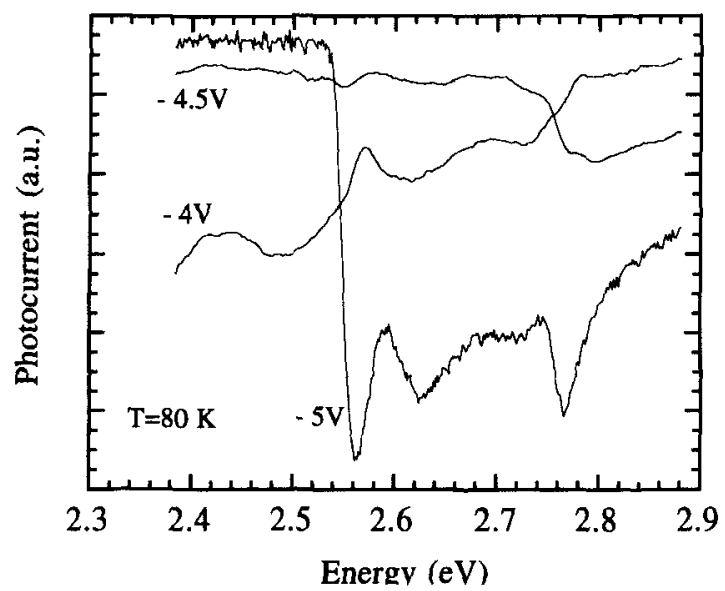

FIG. 21. Sign-reversal of the photocurrent occurring around the breakdown voltage in a $\mathrm{Zn}_{0,9} \mathrm{Cd}_{0.1} \mathrm{Se} / \mathrm{ZnSe} p-i-n$ structure.

when the mesa structures are fabricated. This is again a consequence of the reduction of the surface recombination, which allows a good carrier transport, independent of the transit time effects in the intrinsic region.

\section{Temporal Evolution of the Excitonic Transitions}

In this section we discuss the transient properties of excitons confined in $\mathrm{ZnSe}$-based quantum wells. In optical experiments excitons are formed with some excess energy by the off-resonant pumping. The excess energy is relaxed in a few hundreds fs by the exciton-LO phonon interaction, resulting in a quasi-equilibrium distribution of thermalized excitons. After this short transient, excitons undergo a number of different interactions, namely localization at potential fluctuations, scattering with electrons or other excitons, and eventually recombine radiatively on a time scale of the order of few hundred ps.

The femtosecond dynamics of excitons has been measured in $\mathrm{ZnSe} / \mathrm{ZnSSe}$ (Stevens et al., 1994; Kuroda et al., 1994) and in ZnCdSe/ZnSe (Ding et al., 1993; Neukirch et al., 1994; Tokizaki et al., 1994) heterostructures. The main features of the transient pump and probe transmission spectra are the small blue shift of the excitonic resonance occurring $2-3 \mathrm{ps}$ after the excitation, due to the hard-core exciton repulsion (exchange effect), and the presence of induced absorption bleaching within the inhomogeneously broadened ex- 


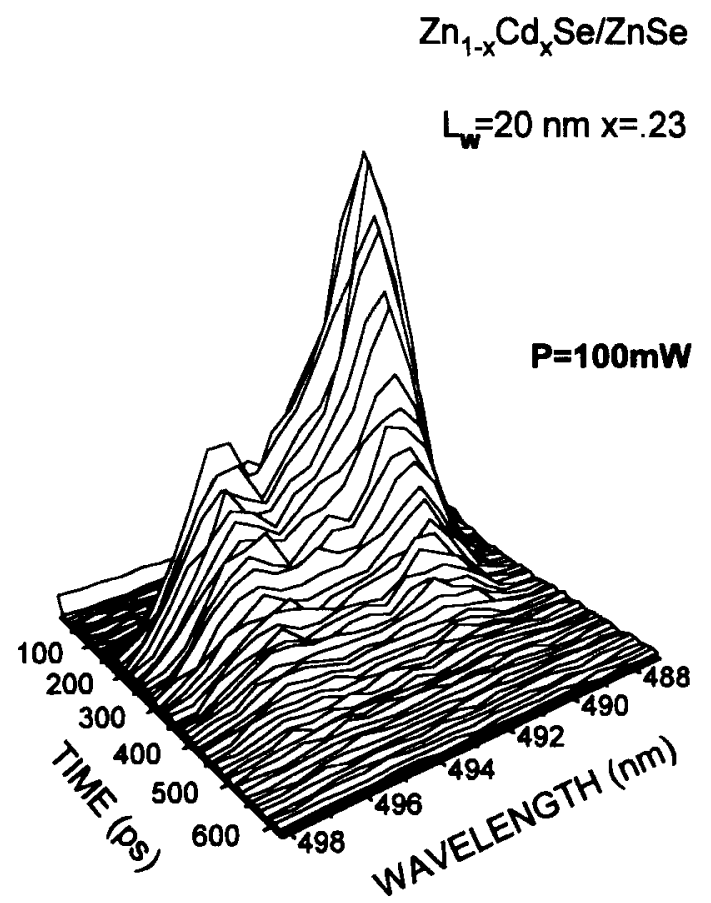

Fig. 22. Time-resolved luminescence spectrum of a $\mathrm{Zn}_{0.77} \mathrm{Cd}_{0.23} \mathrm{Se} / \mathrm{ZnSe}$ multiple quantum well with $L_{w}=20 \mathrm{~nm}$ at $10 \mathrm{~K}$. The integrated power was $100 \mathrm{~mW}$ (excitation wavelength $390 \mathrm{~nm}$, pulse width $2 \mathrm{ps}$, repetition frequency $82 \mathrm{MHz}$ ). The $n=1$ and $n=2$ exciton bands can be clearly resolved around 495 and $491 \mathrm{~nm}$, respectively.

citon linewidth. In $\mathrm{ZnCdSe} / \mathrm{ZnSe} \mathrm{MQWs}$ the typical exciton-phonon interaction time is found to be of the order of $90 \mathrm{fs}$, whereas intraband relaxation of the photoinjected carriers occurs within 2-3 ps. After that, exciton formation occurs (Tokizaki et al., 1994).

On the time scale of the exciton lifetime the behavior of the II-VI quantum wells does not differ appreciably from that of III-V structures. The exciton dynamics is indeed dominated by the temporal evolution of the polariton states (Andreani et al., 1992; Citrin, 1994). In Figure 22 we display the time-resolved luminescence spectrum of a $\mathrm{ZnCdSe} / \mathrm{ZnSe} \mathrm{MQW}$ structure of well width $20 \mathrm{~nm}$ and Cd content $x=0.23$. The $n=1$ and $n=2$ excitonic subbands are clearly resolved with typical decay times of the order of 200 and 150 ps respectively, consistent with the results of Tsutsumi et al., 1994. Somewhat shorter decay times are measured in similar samples (Gutowsky et al., 1995) probably due to relevant localization problems. 


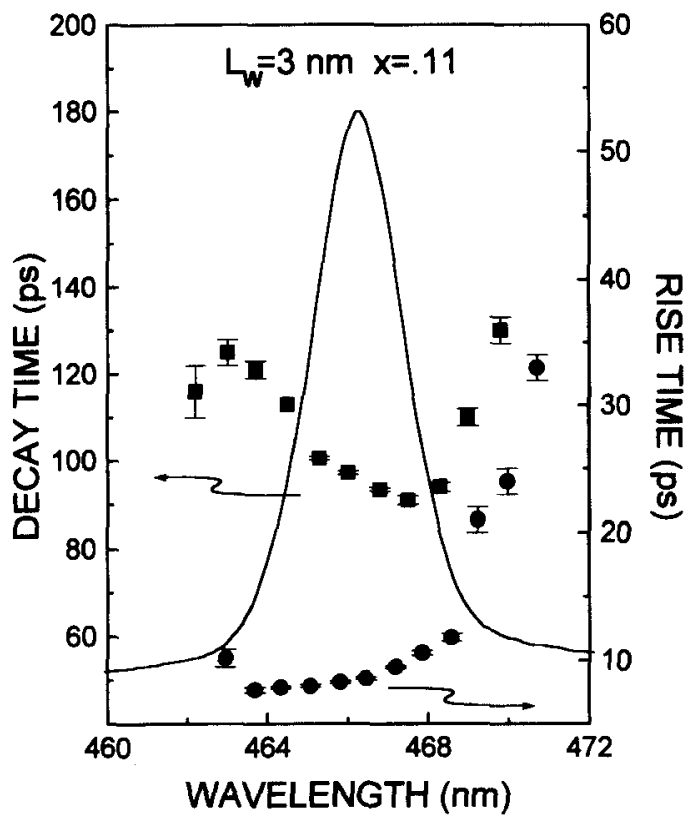

FIG. 23. Spectrally resolved rise time (dots and right-hand scale) and decay-time (squares and left-hand scale) of a $\mathrm{Zn}_{0.89} \mathrm{Cd}_{0.11} \mathrm{Se} / \mathrm{ZnSe}$ multiple quantum well with $L_{w}=3 \mathrm{~nm}$. Note the increase of the rise time occurring in the low-energy tail of the exciton band due to trapping and localization.

The time-resolved luminescence experiments provide important information on the localization of excitons within the inhomogeneously broadened density of states caused by disorder in the ternary alloy $\mathrm{ZnCdSe}$ matrix. As a general trend the temporal evolution of the luminescence from localized excitons exhibits rise time and decay time longer than the free exciton. This is clearly seen in the low-energy tail of the exciton resonance of $\mathrm{ZnCdSe} /$ ZnSe quantum wells under above-barrier excitation (Fig. 23). The long rise time indicates the trapping of excitons at local potential fluctuations. This temporal evolution changes with the excitation intensity, as shown in Figure 24 . With increasing the power density both the rise time and the decay time get shorter, as expected for the free exciton luminescence. This change reveals the saturation of the available density of localization states and the recovery of the intrinsic free-exciton recombination. A further increase of excitation generates a rather high carrier density resulting in the almost

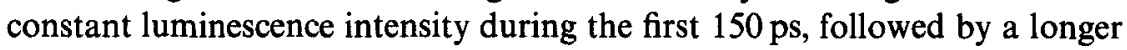
decay time. Under this condition the excitonic population is fed by the carrier plasma as long as excess carriers are available to form new excitons, 


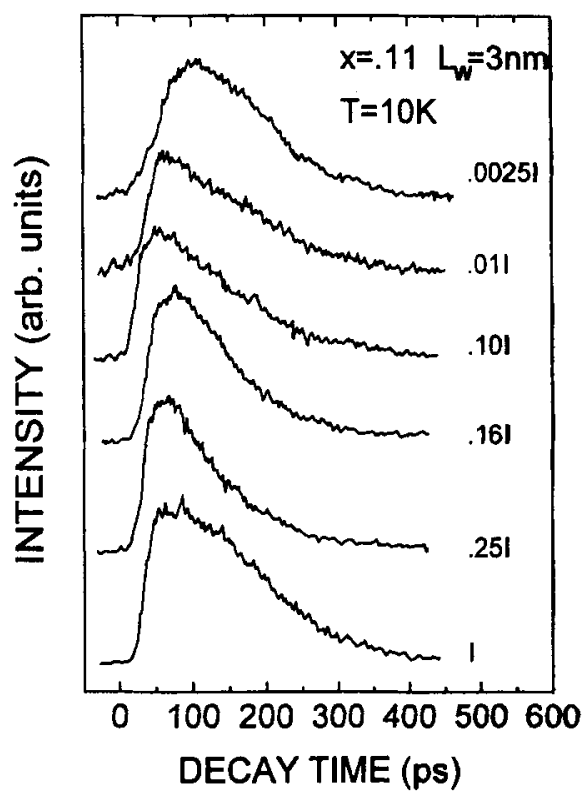

FIG. 24. Temporal evolution of the photoluminescence intensity in a $\mathrm{Zn}_{0.89} \mathrm{Cd}_{0.11} \mathrm{Se} / \mathrm{ZnSe}$ of well width $L_{w}=3 \mathrm{~nm}$ at $10 \mathrm{~K}$. The detection wavelength was fixed at the peak of the exciton band. The maximum integrated power is $\mathrm{I}=80 \mathrm{~mW}$.

resulting in the "plateau" region on the time-resolved curve. The important point is that the localization dynamics is found to change with the quantum well stoichiometry in ternary alloy $(\mathrm{ZnCdSe} / \mathrm{ZnSe})$ quantum wells. Cd-rich samples suffer important compositional and strain fluctuations which are clearly evidenced by the stronger excitonic-absorption linewidth as compared to shallow QWs. Therefore, the recover of the intrinsic free-exciton recombination, monitored through the shortening of the rise and decay time, is often not observed in deep ZnCdSe MQWs. This is clearly shown in Figure 25 where we compare the intensity dependence of the spectrally resolved decay and rise time of $\mathrm{ZnCdSe} \mathrm{MQWs}$ of different composition. As already discussed in Figure 24, in the shallow quantum well the short rise time characteristic of the free-exciton recombination is recovered by increasing the power density (Fig. 25a). On the contrary, a clear localization dynamics is observed in the whole excitation intensity range for the deep quantum wells (Fig. 25b). A detailed rate-equation analysis of the time resolved luminescence traces provides the density of localization centers existing in the quantum well (Lomascolo et al., 1996). This is found to vary between $9.10 \mathrm{~cm}^{-2}$ and $4.10^{10} \mathrm{~cm}^{-2}$ for $\mathrm{Cd}$ content in the range 

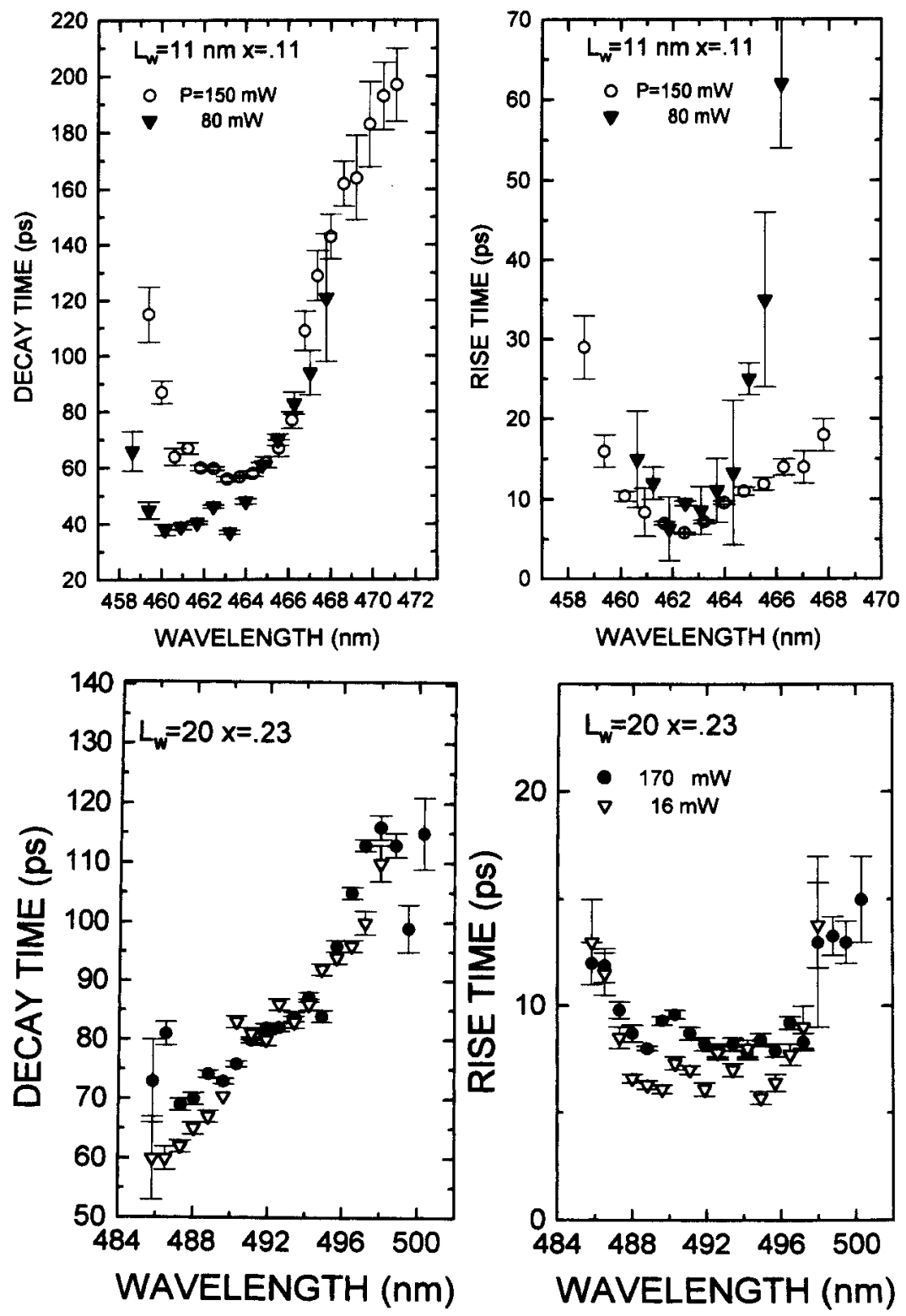

FIG. 25. (a) Intensity dependence of the decay time (left panel) and rise time (right panel) measured from a $\mathrm{Zn}_{0.89} \mathrm{Cd}_{0.11} \mathrm{Se} / \mathrm{ZnSe}$ heterostructure of well width $L_{\mathrm{w}}=11 \mathrm{~nm}$ at $10 \mathrm{~K}$ under different excitation power. Note the shortening of the rise time, indicating the saturation of the localization centers occurring at high photogeneration rates. (b) The same as in Figure 25a, but for a $\mathrm{Zn}_{0.77} \mathrm{Cd}_{0.23} \mathrm{Se} / \mathrm{ZnSe}$ multiple quantum well of width $L_{w}=20 \mathrm{~nm}$. No saturation of the localization centers is observed under intense photoexcitation. 

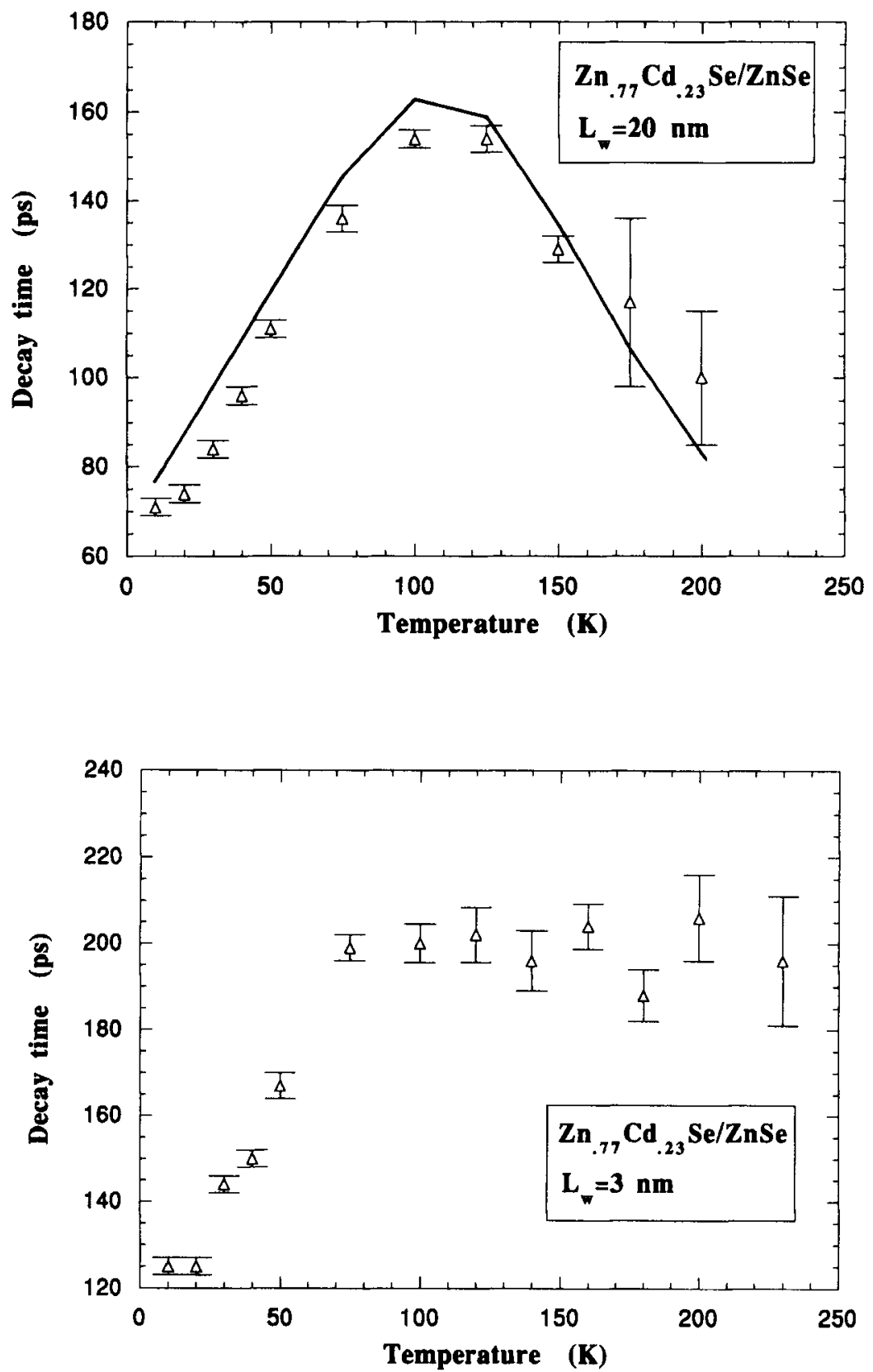

FIG. 26. (a) Temperature dependence of the exciton decay time in $\mathrm{Zn}_{0.89} \mathrm{Cd}_{0.11} \mathrm{Se} / \mathrm{ZnSe}$ multiple quantum wels $\left(L_{w}=7 \mathrm{~nm}\right)$. The curve is calculated according to Eq. 14. (b) Same as in Figure 26a, but for a $\mathrm{Zn}_{0.77} \mathrm{Cd}_{0.23} \mathrm{Se} / \mathrm{ZnSe}$ multiple quantum well sample. 
$0.1<\mathrm{x}<0.23$. This result is very important for the understanding of the lasing mechanism. In fact, stimulated emission is found to occur in the sample of Figure 25b, at injection densities for which the localization of excitons is still dominant. This supports the model of optical gain from localized exciton states proposed by Ding et al. (1992). Conversely, the lasing threshold of the shallow quantum wells (Fig. 25a) occurs at intensity such that the localization is saturated, indicating that the exciton localization is not the main lasing mechanism in shallow ZnCdSe MQWs (Cingolani et al., 1994a). Similar conclusions are found to be valid for the $\mathrm{ZnSe} / \mathrm{ZnSSe}$ heterostructures (Stevens et al., 1994). In this case the localization of excitons primarily occurs at the barrier/well interfaces due to the formation of extended islands.

The temperature dependence of the decay time of the exciton luminescence provides more information on the polariton confinement in the quantum well. The polariton lifetime in quantum wells is expected to grow linearly with the temperature (Andreani et al., 1992; Citrin, 1994). This phenomenon competes with the thermal escape of carriers from the quantum wells which reduces the overlap of the electron and hole wavefunctions, resulting in a decrease of the exciton lifetime. This dynamics is shown in Figure 26 for deep and shallow $\mathrm{ZnCdSe} / \mathrm{ZnSe} \mathrm{MQWs}$. In the shallow quantum well the decay time increases almost linearly with the temperature, then it saturates and starts to decrease around $100 \mathrm{~K}$. At this temperature the less confined particle, presumably the heavy-role, is thermally activated above the barrier and the exciton itself becomes unstable. In the deep quantum well the saturation is observed, but the depth of the quantum well is large enough to prevent thermal escape of the carriers up to room temperature. This spectral behavior has been observed in III-V quantum wells, and has been quantitatively explained by Michler et al. (1992) and by Gurioli et al. (1992). The temperature-dependent exciton decay time is described by

$$
\tau(T)=\frac{\tau_{w}}{1+\frac{\tau_{w}}{\tau_{b}} C^{1 / 2} \exp \left(-\frac{\Delta E}{k T}\right)}
$$

where $C$ is the scattering rate, $\tau_{w(b)}$ is the decay time in the well (barrier), and $\Delta E$ is the activation energy of the carriers confined in the well (this is analogous to the work function of the heavy-hole in the investigated structures). The calculated temperature-dependent decay times are compared to the experimental data in Figure 26 (continuous lines). The activation energy obtained from the fit compares quite well with the potential step experienced by the heavy-holes, thus confirming the offset and the strain correction adopted for the evaluation of the quantized states in these heterostructures (see Section III.1). 


\section{Nonlinear Excitonic Properties}

\section{Basic Theoretical Concepts}

In this section we discuss the nonlinear optical properties of excitons in II-VI quantum wells. Due to the enhanced excitonic stability, nonlinear optical processes involving excitons are very important in II-VI quantum wells and must be considered relevant even for the design of optoelectronic devices operating with high carrier density and at room temperature. The excitonic nonlinearities originate from different mechanisms, namely: (i) the interaction of excitons with an external electric field and (ii) the many-body interactions (Schmitt-Rink et al., 1989). The former case is based on the charge separation occurring in a polarized quantum well, which causes a decreased overlap of the electron-hole wavefunction (quantum confined Stark effect). This in turn causes a reduction of the exciton oscillator strength, and of the binding energy. There are only a few experiments reported in the literature due to the technological problems to be faced in the fabrication of good $p-i-n$ structures (see Section III.3). Electroabsorption experiments have been performed on $\mathrm{ZnSe} / \mathrm{ZnSSe}$ superlattices by Marquadt et al. (1994) who showed a negligible Stark shift of the excitonic resonance. This finding was interpreted as a consequence of the weak exciton polarizability due to the negligible conduction band offset. A distinct quantum confined Stark effect has been revealed under external forward bias either in $p-i-n$ or Schottky diodes embedding $\mathrm{ZnCdSe} /$ $\mathrm{ZnSe} \mathrm{MQWs}$ in the depletion layer. In the former case the built-in electric field was not known, due to the nonohmic contacts and the doping inhomogeneities (Wang et al., 1993a). The Schottky barrier, on the other hand, though giving a large Stark shift and a well-known potential discontinuity (about $1.4 \mathrm{~V}$ for the $\mathrm{Au} / \mathrm{ZnSe}$ interface), results in a worse current-voltage characteristic (Kawakami et al., 1993a; Wang et al., 1994). Low-threshold electroptic modulation recently has been obtained in $\mathrm{ZnCdSe} / \mathrm{ZnSe}$ quantum wells both in photocurrent and luminescence. The bleaching of the oscillator strength, the stark-shift, and the well width dependence of the non-linearity have been quite well described by a variational model for the exciton in electric field (Giugno $e t$ al., 1996). Finally, bistable self-electro-optic operation (Wang et al., 1993b) has been demonstrated in $\mathrm{ZnCdSe} / \mathrm{ZnSe}$ quantum wells.

Many-body nonlinearities, on the other hand, have been extensively studied in recent years, with special attention to III-V heterostructures. In general, the presence of a dense carrier/exciton plasma influences the excitonic states through the screened Coulomb potential and the exchange and correlation interactions which reduce the exciton binding energy and change the self-energy of the electron-hole pair. These many-body processes are treated by means of complicated theoretical models based on the 
random phase approximation, which allow one to describe the carrier density dependence of the electronic and excitonic states (Klingshirn and Haug, 1981; Schmitt-Rink et al., 1989; Haug and Schmitt-Rink, 1985). Most of these theories have been developed for III-V semiconductors, in which excitons are not strongly bound (typical binding energy of the order of $10 \mathrm{meV}$ ). Consistent with the experimental observations, it is found that at carrier densities well below $10^{11} \mathrm{~cm}^{-2}$ the excitonic resonances are bleached and the optical absorption is due to interband transitions. In terms of basic thermodynamics, the free-carrier gas causes a rapid ionization of the exciton gas, and the two phases can coexist only at low carrier densities and temperatures (Cingolani and Ploog, 1991).

In II-VI semiconductors, and especially in quantum wells with enhanced excitonic stability, the coexistence of the exciton and free carrier gas occurs on a wider density and temperature range. Due to the large exciton binding energy, carrier densities in excess of $10^{11} \mathrm{~cm}^{-2}$ can easily be achieved in the material without relevant excitonic bleaching. This has a tremendous impact on the operation principle of optoelectronic devices based on wide band gap II-VI quantum wells.

Many-body processes like electron-electron, exciton--electron, and exciton-exciton interactions, cause the reduction of the exciton binding energy, due to the renormalization of the electronic and excitonic states, and the saturation of the excitonic absorption (exciton bleaching). The exact calculations of these processes are very difficult. In this section, we will discuss a few relevant perturbative approximations, valid in the limit of intermediate carrier densities, which provide handable analytical formulas for the evaluation of the self-energy corrections of the interacting freeexciton/free carrier gas (Cingolani et al., 1996a).

The macroscopic excitonic bleaching occurring at high carrier densities is explained by the reduction of the overlap of the electron and hole wavefunctions caused by the surrounding interacting carriers (Schmitt-Rink et al., 1989). The exciton oscillator strength depends on the electron carrier density in the well, and is proportional to the squared amplitude of the exciton wavefunction in the volume where the electron and the hole overlap. It is expected that the overlap amplitude of the electron and hole rapidly decreases as the sheet carrier density increases. The bleaching of the oscillator strength $f$ is described by a simple saturation model (Schmitt-Rink et al., 1985)

$$
f(n)=f(0) \cdot \frac{1}{1+\frac{n}{N_{c}}}
$$

where $n$ is the total carrier density and $f(0)$ is the unperturbed oscillator strength. $N_{c}$ is a critical saturation density, i.e., the carrier density at which 
$f(n)$ becomes one-half of the value of the unperturbed crystal. This quantity has to be calculated by a full many-body treatment of the various carrierexciton interactions. A useful analytical approximation valid in the nondegenerate limit and at low temperature, including the effect of phase space filling (PSF) and exchange (EXC), has been presented by Schmitt-Rink et al. (1985)

$$
\frac{1}{N_{c}}=\frac{1}{N_{c}^{\mathrm{PSF}}}+\frac{1}{N_{c}^{\mathrm{EXC}}}
$$

where $N_{c}^{\mathrm{PSF}}=\frac{1}{8 \pi a_{0}^{2}}$ and $N_{c}^{\mathrm{EXC}}=\frac{1}{9.89 \pi a_{0}^{2}}$.

Both these terms originate from the Pauli exclusion principle. The PSF describes the blocking of the conduction and valence states available for the formation of new excitons. The short-range exchange interaction represents the energy gained by electrons with same spin orientation in avoiding each other. Long-range Coulomb interactions, i.e., the classical screening among carriers is neglected, because it has been shown to be substantially reduced in quantum well structures with respect to the bulk.

The reduction of the exciton binding energy $\left(E_{b}\right)$ originates from the renormalization of the electron-hole pair energy. At first order we can write a density-dependent exciton binding energy

$$
E_{b}\left(n_{t o t}\right)=E_{b}-B G R\left(n_{e h}\right)-\pi a_{0}^{2}\left(3.84 n_{x}+13.1 n_{e h}\right) E_{b}
$$

In Eq (17) $n_{t o t}=n_{e h}+n_{x}$ indicates the total density of electron-hole pairs (injected or photogenerated) which consists of free carriers $\left(n_{e h}\right)$ and excitons $\left(n_{x}\right)$, distributed according to a temperature- and density-dependent phase diagram. The right hand part of $\mathrm{Eq}$ (17) contains the energy corrections due to the different many-body interactions. BGR is the band gap renormalization induced by free carriers and is discussed in more detail below. The last term includes the blue-shift of the exciton resonance induced by the exciton-exciton interaction, which depends only on the exciton density $n_{x}$ (Schmitt-Rink et al., 1985), and the effect of free carriers on the exciton wavefunction (phase space filling). Actually an additional term accounting for the renormalization of the single particle states induced by the excitons should be included (Cingolani et al., 1996a). For sake of simplicity we will neglect this term in the following, and we will calculate the $B G R$ for the total carrier density, no matter whether the electron-hole pairs are bound or not. These analytical approximations are strictly valid in the limit of nondegenerate Fermi gas and for $k T / E_{b}<1$. These conditions are reasonably fulfilled in II-VI quantum wells under strong photogeneration rates, by virtue of their large effective masses and binding energy. The band gap 
renormalization term consists of the exchange $\left(\Sigma^{\mathrm{exc}}\right)$ and Coulomb-hole $\left(\Sigma^{e h}\right)$ self-energy corrections. According to Campi and Coriasso (1995)

$$
\Sigma^{e x c}=\frac{e \hbar^{2}}{2 a_{o} \mu} \cdot\left[\chi\left(\frac{\chi}{k_{F}+\chi}\right)+k_{F}\right]
$$

where $k_{F}=\left(2 \pi n_{e n}\right)^{1 / 2}$ is the Fermi wavevector, $\mu$ is the electron-hole reduced mass and $a_{0}$ is the quasi-two-dimensional exciton Bohr radius. For the Coulomb-hole term we have

$$
\Sigma^{c h}=\frac{e \hbar^{2}}{2 a_{o} \mu} \cdot \chi
$$

In Eqs (18) and (19) $\chi$ represents the screening wavevector (inverse screening length), given by

$$
\chi=\chi_{e}+\chi_{h}=\frac{2 \pi e^{2}}{\varepsilon} \cdot\left(\frac{\partial n_{e h}}{\partial E_{F}^{e}}+\frac{\partial n_{e h}}{\partial E_{F}^{h}}\right)
$$

where $E_{F}^{e, h}$ is the Fermi energy of electrons and holes. The total band gap renormalization for the neutral electron and hole plasma is thus given by $\operatorname{BGR}\left(n_{e h}\right)=2 \Sigma^{\mathrm{exc}}+{ }^{2} \Sigma^{c h}$, where the factor 2 accounts for the mutual electron-hole and hole-electron interactions. Similarly, the band gap renormalization for the single component (electron or hole) plasma is given by one-half of the BGR obtained for the neutral electron-hole plasma.

Experimentally, one can investigate these processes either by injecting optically or electrically a dense neutral electron-hole plasma in the quantum well, or by using a single component plasma created by the modulation doping in the barrier (either $p$ - or $n$-type). In the former case a twocomponent plasma (TCP) is formed, whereas the latter case allows one to obtain a one-component plasma (OCP) in equilibrium conditions in the well.

\section{Excitons and the One-component Electron Plasma}

The one-component plasma is formed by introducing donor or acceptor atoms in the middle of the barrier during the growth. In order to keep the Fermi level constant through the whole material, the impurity atoms are 

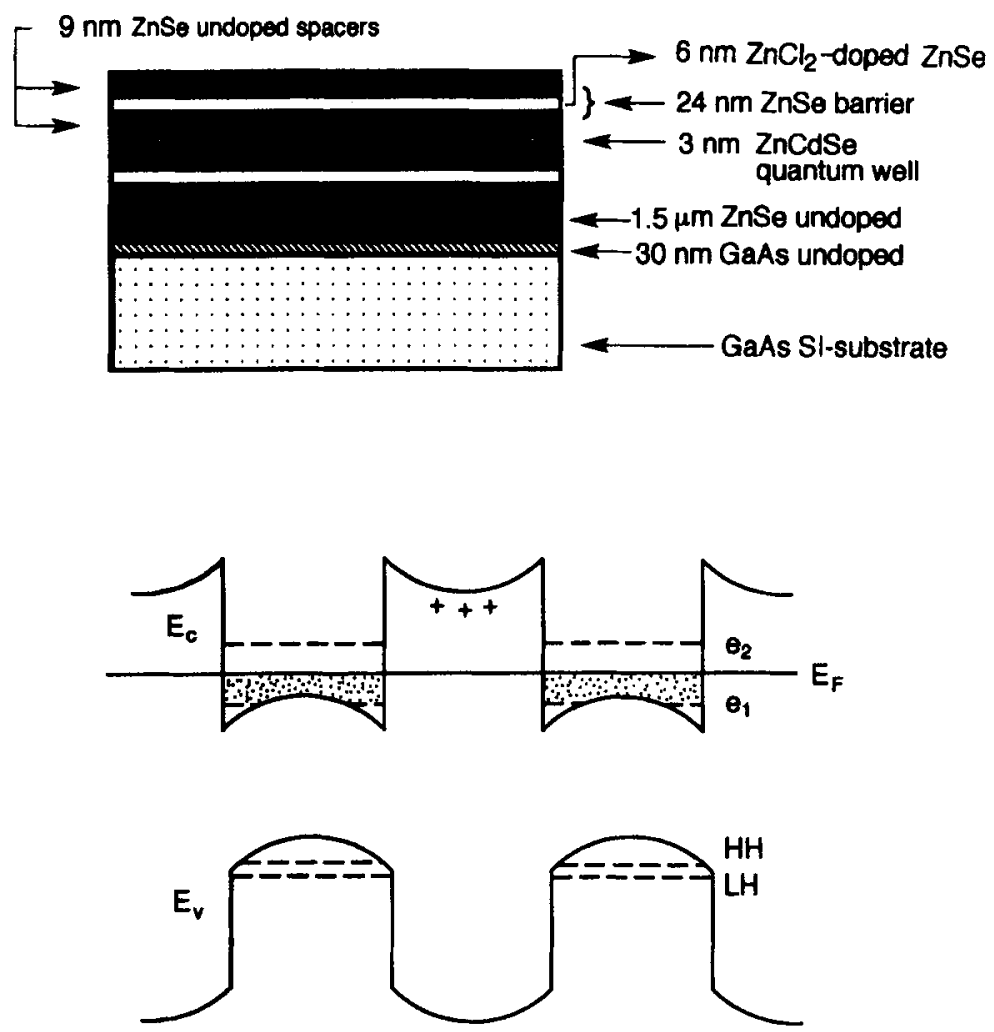

Fig. 27. Layer sequence and band profile of the modulation-doped quantum well heterostructures.

ionized and the free carriers transfer into the well, where the plasma forms (Livescu et al., 1988; Cingolani et al., 1990). The band structure of the undoped material is modified by the electric field created by the charge transfer. The typical structure of the modulation-doped quantum wells (MDQWs) and the corresponding band diagram are shown in Figure 27.

The unperturbed eigenstates of the modulation-doped QWs are modified by the free carriers introduced by the doping. The modified energy levels can be evaluated by solving self-consistently the Poisson and Schrödinger equations. A parabolic bending of the bands, proportional to the sheet carrier density $n$ and to the dielectric constant of the material, is assumed in the calculations. In first-order perturbation theory, the correction to the energy levels induced by the electric field generated by the carriers depends on the band bending and on the electron and hole wavefunctions. This in turn affects the excitonic states. In ZnSe-based quantum wells the electro 

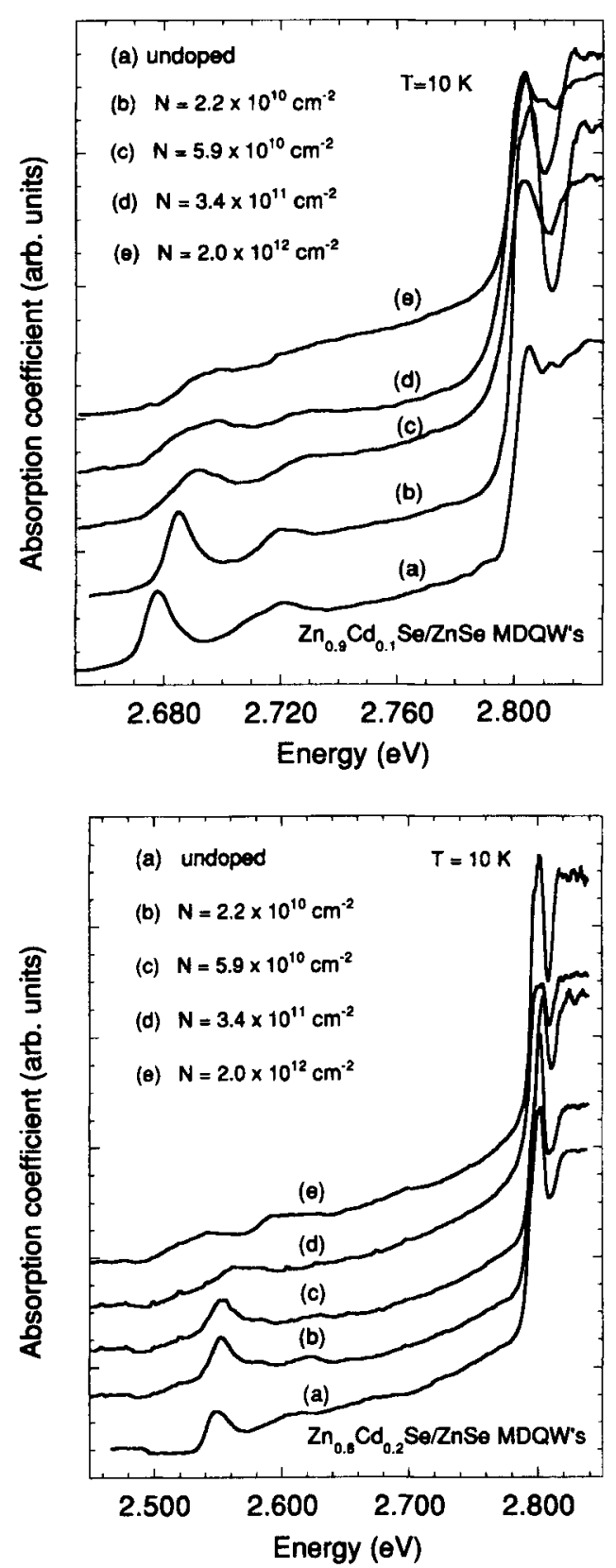

Fig. 28. (a) Absorption spectra of a set of $\mathrm{Zn}_{0.9} \mathrm{Cd}_{0.1} \mathrm{Se} / \mathrm{ZnSe}$ modulation-doped multiple quantum wells with different doping concentrations. The well width is $L_{w}=3 \mathrm{~nm}$. (b) The same as in Figure 28a, but for a set of $\mathrm{Zn}_{0.8} \mathrm{Cd}_{0.2} \mathrm{Se} / \mathrm{ZnSe}$ modulation doping quantum wells. 
static effects on the ground level heavy-hole exciton turn out to be very small, of the order of 1-2 meV at carrier densities of the order of $10^{12} \mathrm{~cm}^{-2}$. We will therefore neglect this correction in the following discussion. Figure 28a shows the low temperature absorption spectra of $n$-type $\mathrm{Zn}_{0.9} \mathrm{Cd}_{0.1} \mathrm{Se}$ MDQWs as a function of the sheet carrier density from $2.2 \cdot 10^{12} \mathrm{~cm}^{-2}$ (Calcagnile et al., 1996). Curve (a) refers to the undoped sample and is shown as reference. This spectrum exhibits the heavy-hole (HH) and light-hole (LH) excitonic transitions, with full width at half maximum (FWHM) of $11 \mathrm{meV}$ and $20 \mathrm{meV}$, respectively. Moreover, at $2.805 \mathrm{eV}$ the excitonic transition from the undoped $\mathrm{ZnSe}$ barrier layer is also observed. The absorption spectra of the modulation-doped samples [curves (b)-(d)] exhibit a clear reduction of the oscillator strength. At the highest density, the spectrum labeled (e) does not show any excitonic feature indicating that the carrier concentration in this sample is above the critical density for the exciton bleaching. Similar results are obtined from the deeper $\mathrm{Zn}_{0.8} \mathrm{Cd}_{0.2} \mathrm{Se} /$ $\mathrm{ZnSe} \mathrm{MQWs}$ shown in Figure 28b, though with a stronger excitonic resonance. At the maximum sheet carrier density $\left(n=2.0 \cdot 10^{10}\right.$ to $2.0 \cdot 10^{12} \mathrm{~cm}^{-2}$ ) still a weak excitonic peak is observed in the spectrum, as expected from the enhanced exciton binding energy ( $37 \mathrm{meV}$ for the $x=0.2$ sample and $30 \mathrm{meV}$ for the $x=0.1$ sample, respectively).

The effect of carrier concentration on the intensity of the optical transitions can be evaluated by estimating the oscillator strength and the binding energy of the $\mathrm{HH}$ exciton. The oscillator strength is directly proportional to the integrated area of the absorption peak (Masumoto et al., 1985):

$$
\int \alpha(E) d E=\frac{2 \pi^{2} e^{2} \hbar}{m c \varepsilon^{1 / 2}} f(0)
$$

where $e$ is the electron charge, $\varepsilon$ is the dielectric constant, and $c$ the light velocity. Using $\mathrm{Eq}(21)$ the $\mathrm{HH}$ oscillator strength can be obtained directly from the experimental spectra of Figure 28. As shown in Figure 29 the oscillator strength of the heavy-hole exciton decreases with increasing the sheet carrier density. The experimental results follow the saturation behavior of the exciton oscillator strength predicted by Eq (16). The critical density for exciton bleaching is found to be $N_{c}=1.0 \cdot 10^{11} \mathrm{~cm}^{-2}$ for the shallow MDQWs, and $N_{c}=2.0 \cdot 10^{11} \mathrm{~cm}^{-2}$ for the $x=0.2 \mathrm{MDQWs}$. These values of $N_{c}$ compare reasonably well with the theoretical critical densities obtained by the perturbative description $\mathrm{Eq}$ (15), which amount to $2 \cdot 10^{11} \mathrm{~cm}^{-2}$ and $3.6 \cdot 10^{11} \mathrm{~cm}^{-2}$ for $x=0.1$ and $x=0.2$, respectively. We should mention that the above saturation model has been obtained for the two-component electron-hole plasma. The straightforward extension to 

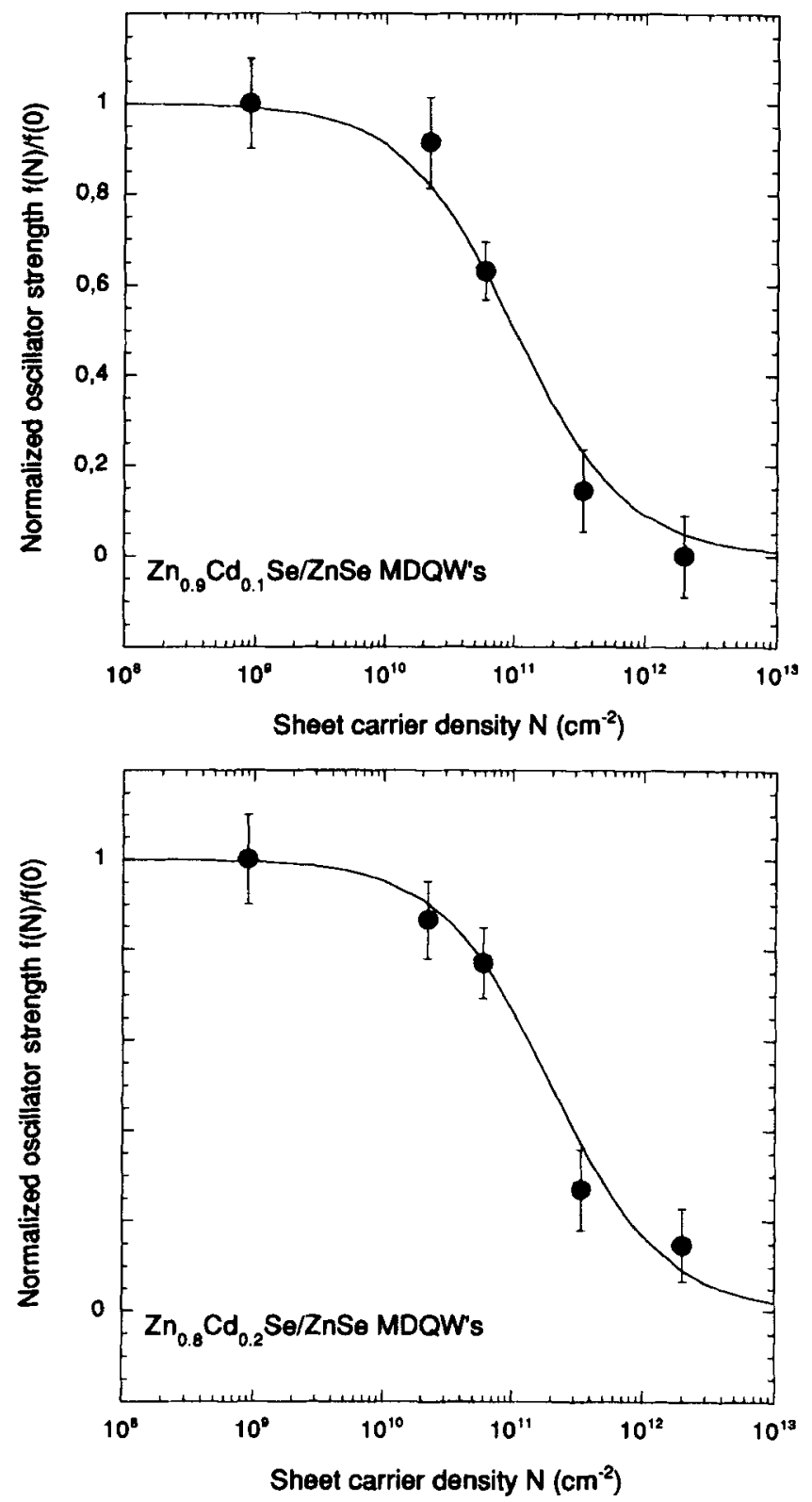

FiG. 29. (a) Carrier density dependence of the normalized integrated absorption of the heavy-hole exciton peak for the $\mathrm{Zn}_{0.9} \mathrm{Cd}_{0.1} \mathrm{Se} / \mathrm{ZnSe}$ modulation-doped multiple quantum wells of Figure 28a. The continuous curve is the saturation of the oscillator strength calculated by means of Eq. (16). (b) The same as in Figure 29a but for the set of $\mathrm{Zn}_{0.8} \mathrm{Cd}_{0.2} \mathrm{Se} / \mathrm{ZnSe}$ modulation-doped multiple quantum wells studied in Figure $28 \mathrm{~b}$. 

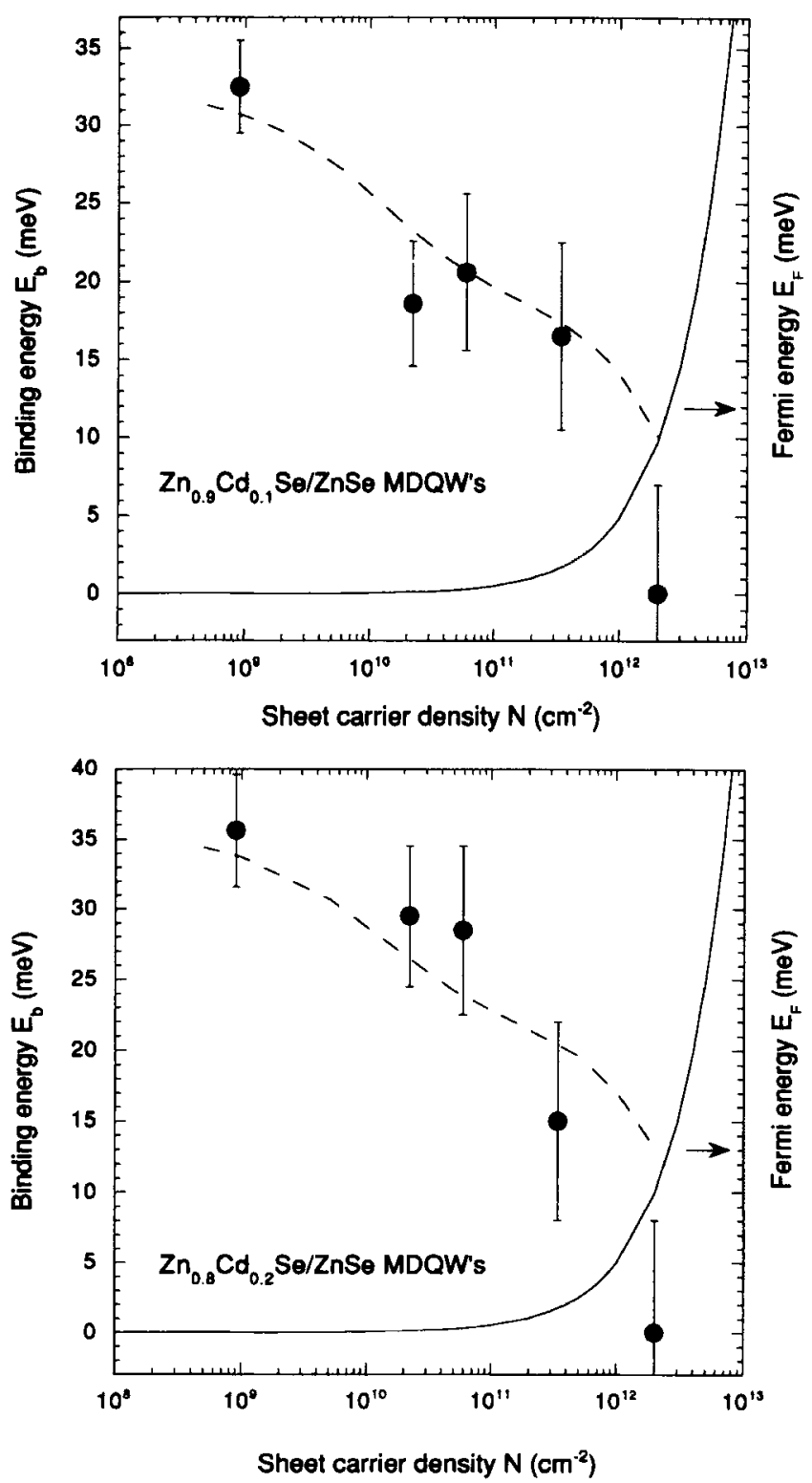

FIG. 30. (a) Carrier density dependence of the heavy-hole exciton binding energy (symbols) obtained from the $\mathrm{Zn}_{0.9} \mathrm{Cd}_{0.1} \mathrm{Se} / \mathrm{ZnSe}$ modulation-doped multiple quantum wells of Figure 28a. The dashed curve represents the renormalization of the exciton binding energy calculated by means of Eqs (18)-(20). The continuous curve is the calculated low-temperature quasiFermi energy of the electron plasma. The increasing error bars are due to the enhancement of the spectral broadening occurring at high carrier density which affects the line-shape fitting precision. (b) The same as in Figure $30 \mathrm{a}$ but for the $\mathrm{Zn}_{0.8} \mathrm{Cd}_{0.2} \mathrm{Se} / \mathrm{ZnSe}$ modulation-doped multiple quantum wells of Figure $28 \mathrm{~b}$. 
the one-component electron-plasma existing in MDQWs can thus be questionable.

The effects of carrier density on the HH exciton binding energy were determined by means of a line-shape analysis (see Section III.1), through the evaluation of the energy difference between the HH exciton energy and its continuum. The experimental results are shown in Figure 30. The curves were calculated assuming that the binding-energy reduction is caused by the band gap renormalization induced by the single-component plasma, neglecting excitonic interactions, i.e. $E_{b}^{\prime}(n)=E_{b}-\mathrm{BGR}$, where $\mathrm{BGR}=\Sigma^{\text {exc }}+\Sigma^{\text {ch }}$ is given by Eqs (18) and (19). The overall agreement is good at low and intermediate densities, where both the theory and the line shape fitting of the spectra are rather precise. At high carrier densities the agreement becomes poor. This is expected since the binding energy obtained from the line shape fitting is rather unprecise due to the broadening of the spectra (see the large error bar). Also, the analytical expressions for the self-energies become unreliable in the high density limit.

\section{Excitons and the Electron - hole Plasma}

In this section we discuss the nonlinear properties of excitons in the presence of a dense neutral electron-hole plasma. The basic physics is analogous to the OCP case. However, under stationary conditions, excitons can form and coexist with free carriers leading to a complicated free carrier/exciton phase boundary. The key-parameter is the exciton binding energy modified by the many-body interactions and by the finite temperature of the distribution functions, which governs the dissociation of the exciton phase into an electron-hole plasma phase. Depending on the degree of confinement of the exciton wavefunction, identical injection conditions can create either an exciton-rich or a free-carrier-rich phase. This in turn determines the main stimulated emission mechanism in quantum wells of different size and composition. The most direct way to study these phenomena is the nonlinear pump and probe transmission spectroscopy, in which a strong pump beam creates the electron-hole plasma while a weak tunable beam probes the changes of the excitonic absorption.

In Figure 31, we display the intensity dependence of the pump and probe transmission spectra measured at $10 \mathrm{~K}$ in two samples with exciton binding energies of $25 \mathrm{meV}$ (Fig. 31a) and $38 \mathrm{meV}$ (Fig. 31b) (Cingolani et al., 1994e, 1996a). In both cases a clear exciton bleaching is observed. The bleaching intensity amounts to about $1 \mathrm{~kW} \mathrm{~cm}{ }^{-2}$ for the shallow wells and increases up to about $50 \mathrm{~kW} \mathrm{~cm}^{-2}$ in the deeper wells, having considerably stabler exciton. A quantitative interpretation of these experiments is based on the line-shape 

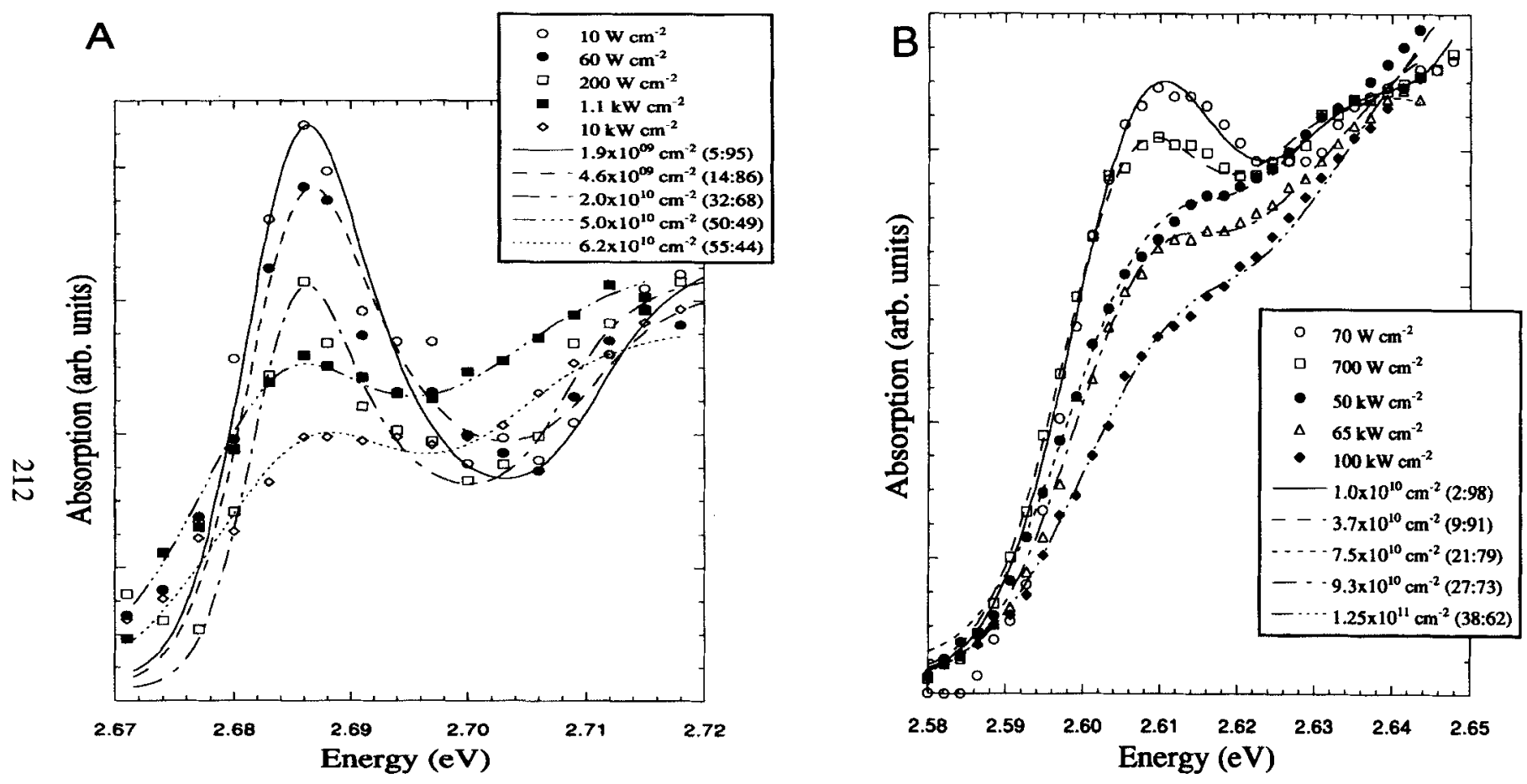

Fig. 31. (a) Nonlinear pump-and-probe absorption spectra measured at $10 \mathrm{~K}$ on a $\mathrm{Zn}_{0.89} \mathrm{Cd}_{0.11} \mathrm{Se} / \mathrm{ZnSe}$ multiple quantum well with $L_{\mathrm{w}}=11 \mathrm{~nm}$ under different pump intensities (symbols). The continuous lines are the calculated nonlinear absorption spectra. For sake of clarity the number of experimental points plotted on the figure has been reduced. The inset shows the total carrier densities corresponding to different pump intensities. In parenthesis we report the ratio of free carriers and excitons obtained from the self-consistent calculation of the pump-and-probe spectra for each pumping condition $\left(n_{e h}: n_{x}\right)$. (b) The same as in Figure $31 \mathrm{~b}$ but for $\mathrm{Zn}_{0.77} \mathrm{Cd}_{0.23} \mathrm{Se} / \mathrm{ZnSe}$ multiple quantum wells of well width $L_{w}=3 \mathrm{~nm}$. 
analysis of the nonlinear pump-and-probe spectra. Following Section IV.1, the nonlinear absorption profile is given by:

$$
\begin{aligned}
& \alpha\left(\hbar \omega, n_{\text {tot }}\right)=\mathrm{const} \cdot\left(\frac{1}{1+\frac{n_{\text {tot }}}{N_{c}}}\right) \cdot G\left\{\hbar \omega-\left[E_{g}-E_{b}^{\prime}\left(n_{t o t}\right)\right], \Gamma\left(n_{t o t}\right)\right\} \\
& \quad+\text { const } \cdot \Theta\left[\hbar \omega-E_{g}^{\prime}\left(n_{e h}, \Gamma\left(n_{t o t}\right), \gamma\right)\right] \cdot S\left(\hbar \omega-E_{b}\right) \cdot f_{e}\left(n_{e h}, T\right)-f_{h}\left(n_{e h}, T\right)
\end{aligned}
$$

In Eq (22) $n_{t o t}$ is the total density of elementary excitations given by the sum of free-carrier and exciton density, distributed according to the temperature dependent phase diagram of the exciton and electron-hole plasma phase. The first factor of Eq (22) accounts for the saturation of the oscillator strength of the excitonic transition, through the critical saturation density $N_{c}$ (Eqs (15) and (16)). The exciton density of states is modeled by a Gaussian function $G$, whose position depends on the renormalized exciton binding energy (Eq (17)). The $\Theta$ function in Eq (22) models the steplike density of states, which includes a phenomenological broadening factor $\gamma$ to account for compositional fluctuations in the ternary well. Coulomb coupling at the continuum, though very weak in these structures, is included by using the Sommerfeld factor $S(\hbar \omega)$. A density-dependent lifetime broadening factor $\left[\Gamma\left(n_{t o t}\right)\right]$ is used in the calculations. Finally, $T$ indicates the thermodynamic temperature of the distribution functions $\left(f_{e}, f_{h}\right)$.

The calculated pump and probe spectra are shown by the continuous lines in Figure 31, showing excellent agreement with the experimental spectra. The exciton bleaching is found to occur at $4 \cdot 10^{10} \mathrm{~cm}^{-2}$ and $1.5 \cdot 10^{11} \mathrm{~cm}^{-2}$ in the two samples, consistent with the different exciton binding energies. These values are considerably smaller than those expected from Eq. (16). This result is mainly due to the finite thermodynamic temperature assumed for the electron-hole and exciton populations and to the complete description of the many-body renormalization of the exciton binding energy included in our model. The equilibrium between the two phases is established by a mass-action law. A set of coupled equations for the total density of photogenerated elementary excitations

$$
n_{\text {tot }}=n_{\text {eh }}+n_{\text {exc }}
$$

and for the relative balance of free carriers and excitons

$$
\frac{n_{e h}^{2}}{n_{\text {exc }}}=\frac{m_{e} m_{h} k_{b} T}{2 \pi \hbar^{2}\left(m_{e}+m_{h}\right)} \cdot \exp \left(\frac{-E_{b}^{\prime}\left(n_{t o t}\right)}{k_{b} T}\right)
$$



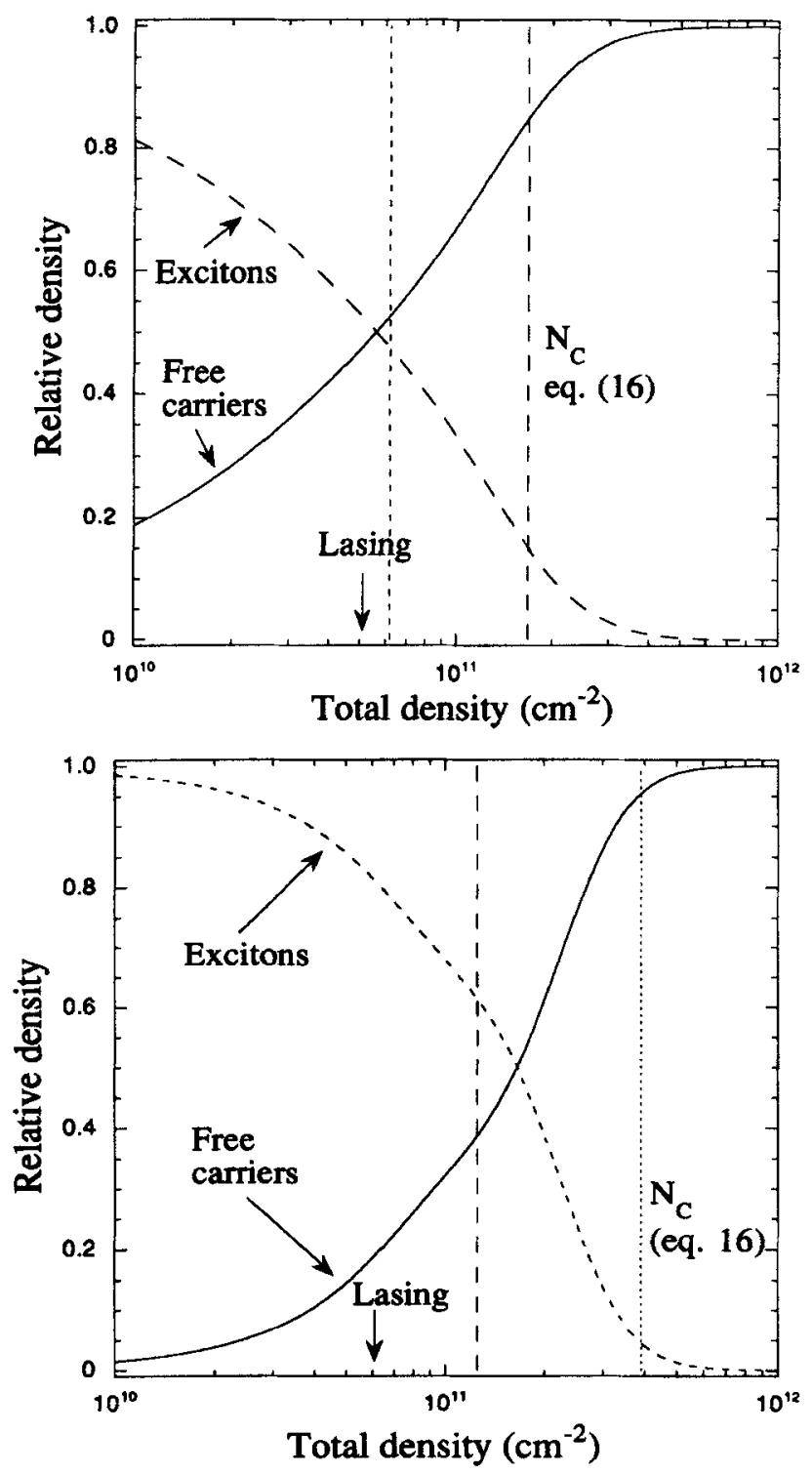

Fig. 32. (a) Phase diagram of the exciton gas and free-carrier gas coexisting in the sample investigated in Figure 31a. The dashed and continuous curves indicate the exciton and free-carrier populations, respectively. The experimental exciton saturation density obtained from the pump and probe line-shape analysis is indicated by the vertical short-dashed line, to be compared with the $N_{c}$ value calculated by means of $\mathrm{Eq}$ (16) (long-dashed vertical line). The carrier density of the lasing threshold (corresponding to $4-6 \mathrm{~kW} \mathrm{~cm}^{-2}$ pump intensity) is indicated by the vertical arrow. Depending on sample characteristics, this falls either in the free-carrier-rich or in the exciton-rich part of the phase diagram. (b) The same as in Figure 32a but for the sample investigated in Figure $31 \mathrm{~b}$. 
has to be solved self-consistently as a function of the total density of elementary excitations $\left(n_{t o t}\right)$. All the many-body renormalizations are taken into account according to $\mathrm{Eq}$ (17).

The results of the calculations are shown in Figure 32 for the same samples investigated in Figure 31 (Cingolani et al., 1996a). The key parameters are the temperature of the distribution function and the total density $n_{t o t}$, which affect the boundary between the free-carrier and exciton phase in a dramatic way. The carrier temperature adopted in Figure 32 was evaluated from the slope of the high-energy tail of the luminescence spectra recorded at different excitation intensities under the photogeneration conditions of the pump and probe experiments. Typical values ranged between $2 \mathrm{meV}$ and $5 \mathrm{meV}$ in the investigated excitation intensity range, and were found to vary sublinearly with the excitation intensity. The phase diagrams of Figure 32 indicate that the transition from an exciton-rich to a freecarrier-rich phase indeed occurs at $n_{\text {tot }}$ values below those expected from the simple approach of Eq. (16), provided the actual carrier temperature and the many-body corrections due to free carriers and excitons are taken into account. In shallow quantum wells the free-carrier phase sets in at injected densities well below $1 \cdot 10^{11} \mathrm{~cm}^{-2}$, consistent with the observation of exciton bleaching in the pump and probe spectra. In deeper wells the exciton phase dominates up to higher $n$-values resulting in a stable exciton gas even under very intense injection rates. This finding has a strong impact on the operation of quantum well lasers based on II-VI materials. In fact, lasing occurs at injected carrier densities which can be either above or below the boundary of the exciton/free-carrier phase transition (resulting in a freecarrier laser or in an excitonic laser), depending on the temperature and on the binding energy of the exciton, i.e. on the compositional and structural parameters of the quantum wells.

\section{Role of Excitons in the Lasing of ZnSe-based Quantum Wells}

In this section we will discuss the interplay between exciton and freecarrier recombination in the stimulated emission mechanism of $\mathrm{ZnCdSe}$ / $\mathrm{ZnSe}$ and $\mathrm{ZnSe} / \mathrm{ZnSSe} \mathrm{MQWs}$. It is well known that the operation principle of a laser is based on a three-level system in which the electronic population of the intermediate level is inverted by the fast relaxation of electrons injected in the topmost level. In semiconductors the three-level system involves the electron and hole quasi-Fermi levels and the renormalized band gap from which stimulated emission occurs. Such process involves free carriers distributed in the conduction and valence bands. In II-VI wide 
band gap quantum wells, the strong Coulomb correlation may lead to stable excitons even in the presence of a dense free-carrier background. Lasing can occur through the stimulated recombination of excitons as well, provided an intermediate level exists to form the three-level system, namely a localized exciton state or the final state of a scattering process (exciton-exciton, exciton-electron, exciton-phonon).

These processes can be distinguished spectroscopically by the characteristic energy of their luminescence. In general, energy and momentum conservation and Boltzman distribution for the excitons are assumed in the scattering process (Klingshirn and Haugh, 1981). For the free excitonexciton scattering the resulting emission should occur at energy:

$$
\hbar \omega_{\mathrm{cxc}-\mathrm{exc}} \simeq E_{H H}-E_{b}-\frac{\hbar^{2} k^{2}}{2 \mu}
$$

where $E_{H H}$ is the free exciton (heavy-hole) energy, and $\mu$ is the reduced exciton mass. If the scattering involves a free exciton and a localized exciton the resulting photon energy can be obtained by replacing the exciton binding energy with the localization energy of the trapped exciton, which is ionized after the scattering. Therefore, the exciton-exciton collision should result in a characteristic emission band which is red-shifted with respect to the free exciton emission by an amount equal to the exciton binding energy (neglecting the kinetic energy).

The exciton-electron scattering should occur at energy

$$
\hbar \omega_{\mathrm{exc}-e l}=E_{\mathrm{HH}}-\frac{\hbar^{2} k^{2}}{2 m_{e}}
$$

As the kinetic energy term can usually be neglected, this recombination mechanism mostly contributes to the broadening of the excitonic line. Eventually, a spectral shift from the excitonic band can be observed with increasing the excitation intensity or the temperature. In particular, an anomalous temperature dependence of the emission is expected, which follows the simple relation (Klingshirn and Haug, 1981):

$$
\hbar \omega(T)_{\mathrm{exc}-e l} \simeq E_{H H}(T)+\sigma\left(\frac{m_{e}}{m_{H H}}\right) K_{b} T
$$

where $\sigma$ is a weak function of the effective mass ratio.

The energy conservation in the exciton-phonon scattering leads to the well-known relation

$$
\hbar \omega_{\mathrm{exc}-\mathrm{LO}}=E_{H H}-\hbar \omega_{\mathrm{LO}}
$$


In $\mathrm{ZnSe}$ and related ternary alloys, the LO phonon energy amounts to about $31 \mathrm{meV}$, resulting in a strongly red-shifted emission band.

We should mention that biexcitons also can play a role in the lasing processes. The formation of biexcitons in II-VI quantum wells has been investigated by few groups ( $\mathrm{Fu}$ et al., 1988; Kuroda et al., 1992a). The reduced binding energy of the biexciton with respect to the exciton, and the competition of inelastic scattering processes make the biexciton particle rather unstable, so that biexciton lasing is very unlikely to be dominant in ZnSe MQWs at high temperatures. Recent observations of biexciton recombination and lasing have been reported by Wang and Simmons (1995), Kreller et al. (1995), Yamada et al. (1995), and Kozlov et al. (1996).

Finally, we recall that the free-carrier recombination is treated by the usual Fermi gas model. The electron and hole population in quasistationary conditions is modeled by Fermi-Dirac distribution functions $\left(f_{e, h}\right)$, taking into account the effective carrier temperature, the band gap renormalization, and the density-dependent quasi-Fermi level $\left(F_{e, h}\right)$ (Lasher and Stern, 1964). Actually, many-body effects and partial removal of the $k$-conservation are included in the PL line-shape according to Section IV.1:

$$
I(\hbar \omega) \simeq \int_{\infty}^{\infty} D_{\text {joint }}(E) f_{e}(E) \cdot\left[1-f_{h}(-E)\right] L\left(\hbar \omega-E_{g}^{\prime}, \Gamma\right) \cdot d E
$$

where $D_{\text {joint }}$ is the joint density of states and $E_{g}^{\prime}$ is the renormalized band gap edge. Under these conditions, the PL is expected to raise around the renormalized band gap edge, with considerable spectral broadening in the low-energy tail; and a bandwidth somewhat proportional to the total quasi fermi level of the electron-hole plasma.

The unambiguous identification of one of the above processes needs a systematic investigation, either in terms of spectral positions or in terms of temperature and intensity dependence. In particular, the free-carrier recombination is expected to red-shift with the carrier density, proportionally to the band gap shrinkage, whereas the other processes exhibit a spectral shift due to the increasing exchange of kinetic energy in the scattering. These spectral features are often difficult to be distinguished, and are strongly affected by the broadening of the emission bands occurring with increasing the excitation power. In order to facilitate the comparison of the experimental spectra with Eqs (25)-(28), we plot in Figure 33 the well width dependence and the intensity dependence of the stimulated emission band measured in a set of $\mathrm{ZnS}_{0.18} \mathrm{Se}_{0.82} / \mathrm{ZnSe}$ superlattices (Cingolani et al., 1996b). The exciton energy determined by photoluminescence excitation or absorption spectroscopy is used as the origin of the energy axis, to determine the relative energy position of the photoluminescence line below and above the stimulation threshold. Two main conclusions can be drawn 


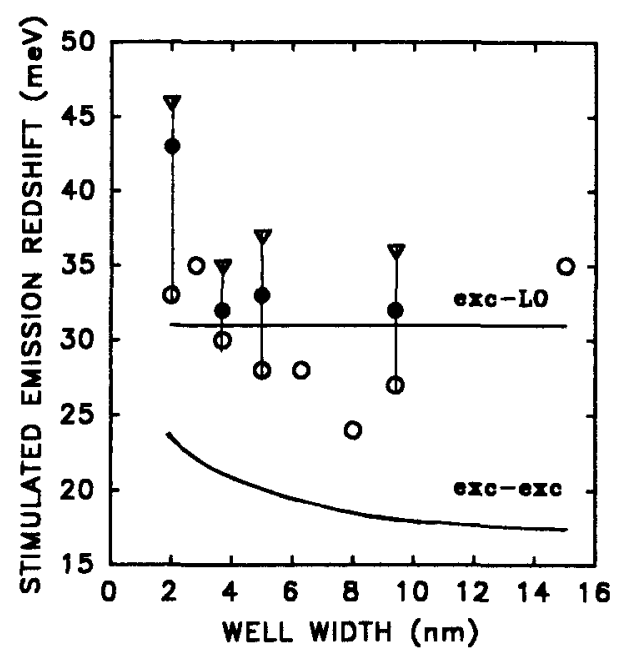

FiG. 33. Well width dependence of the stimulated emission red shift measured in a set of symmetric $\mathrm{ZnS}_{0.18} \mathrm{Se}_{0.82} / \mathrm{ZnSe}$ superlattices at $10 \mathrm{~K}$. The empty circles were measured just at the threshold for the stimulated emission $I_{\sigma}$. The full circles and the triangles correspond to excitation intensities equal to $5 I_{o}$ and $10 I_{o}$ respectively. The curves represent the expected red shift of the recombination due to inelastic exciton-phonon (exc-LO) and exciton-exciton (exc-exc) scattering processes.

from these results: first, the red shift of the stimulated emission around threshold ranges between 20 and $35 \mathrm{meV}$ irrespective of the well width (empty dots in Fig. 33). The measured values compare neither with the characteristic exciton binding energy $\left(E_{b}\right.$, from Fig. (13) nor with the LO phonon energy (LO line) in the superlattices. This rules out the free exciton-exciton [Eq. (25)] or exciton-phonon scattering [Eq (28)] as dominant lasing mechanisms in ZnSe-based MQWs.

Second, the splitting is found to increase considerably with increasing the excitation intensity. This is shown by the different symbols plotted in Figure 33 , representing the red shift of the stimulated emission for excitation intensities close to the stimulation threshold $I_{0}$ (empty dots), at $5 I_{0}$ (full dots) and at $10 I_{0}$ (triangles), as measured in few selected samples. This might explain why, accidentally, particular combinations of sample size and excitation intensity can result in characteristic stimulated emission energies which are apparently consistent with Eqs (25)-(28), thus leading to wrong attributions (Dabbico et al., 1992). We should mention that the absolute position of the stimulated emission is found to depend not only on the temperature and excitation intensity, but also on the physical size of the small cavities cleaved for the high excitation intensity studies. 
The exciton electron scattering process is somewhat more difficult to identify. The main emission band should have a clear onset of the lowenergy tail of the exciton band, and a broad low-energy tail due to the kinetic energy distribution of the scattered electrons recombining after the collision. However, the stimulated emission of the samples investigated in Figure 33 is always observed with a considerable red shift even at the stimulation threshold. This rules out the exciton-electron scattering as dominant recombination mechanism in our samples. Similar results are found for the $\mathrm{ZnCdSe} / \mathrm{ZnSe}$ material system. Based on these considerations, we are forced to deal with the two remaining mechanisms: namely, scattering involving localized excitons and free-carrier recombination. Both processes are likely to be dominant in the ZnSe-based MQWs. The presence of localized excitons is demonstrated by a number of optical experiments, namely photocurrent and PLE spectroscopy, in both $\mathrm{ZnSe} / \mathrm{ZnSSe}$ (Kuroda et al., 1992b) and $\mathrm{ZnCdSe} / \mathrm{ZnSe}$ (Ding et al., 1990b; Cingolani et al., 1995). The possibility of optical gain at the low-energy tail of a strongly inhomogeneous excitonic density of states has been recently discussed in $\mathrm{ZnCdSe} / \mathrm{ZnSe}$ heterostructures with rather high $\mathrm{Cd}$ content (Ding et al., 1992; Ding et al., 1993). On the other hand, electron-hole plasma recombination can be obtained provided the photogeneration rate is strong enough to overcome the Mott threshold for exciton screening.

The spectroscopic identification of lasing from localized excitons or free carriers is very difficult. A systematic comparison of the pump and probe spectra displayed in Figure 31 and the stimulated emission spectra collected from $\mathrm{ZnCdSe} \mathrm{MQWs}$ of different composition and size reveals that stimulated emission threshold (of the order of $4-6 \mathrm{~kW} \mathrm{~cm}^{-2}$ ) can be either larger or smaller than the exciton bleaching intensity, depending on the exciton stability of the investigated sample. Therefore, excitonic lasing or free-carrier lasing can be obtained depending on the phase diagram of the exciton and free-carrier gas in thermal equilibrium. Two limiting cases can be clearly identified: (a) the deep and narrow quantum wells $\left(E_{b}>32 \mathrm{meV}\right)$ exhibit stimulated emission at power densities such that distinguishable excitonic features can still be observed in the pump and probe spectra; (b) lasing in the shallow and thick quantum wells $\left(E_{b}<25 \mathrm{meV}\right)$ occurs at power densities well above the bleaching intensity. In the wide range of intermediate size and composition, the bleaching intensity and the stimulated emission threshold are comparable, preventing a straightforward correlation between the two processes.

An unambiguous identification of these two different recombination mechanisms can be obtained by measuring the diamagnetic shift of the stimulated emission in high magnetic field. The basic idea, which was already successfully applied to GaAs quantum wells (Cingolani et al., 1991), 
relies on the different magnetoluminescence shift exhibited by excitonic and free-carrier-related features in the optical spectra. The excitonic recombination in magnetic field (B) exhibits a diamagnetic shift quadratic in the field:

$$
\Delta E \simeq \frac{\hbar^{4} \varepsilon^{2}}{e^{2} \mu^{3}} \cdot B^{2}
$$

where $\mu$ is the reduced exciton mass. By using the correct in-plane heavyhole mass, a very small diamagnetic shift, of the order of $2-4 \mu \mathrm{eV} / T^{2}$ is expected for $\mathrm{Zn}_{0.9} \mathrm{Cd}_{0.1} \mathrm{Se}$. Conversely, the free-carrier recombination is characterized by the well-known Landau shift, linear in the magnetic field:

$$
\Delta E \simeq \frac{1}{2} \cdot\left[\frac{\hbar e}{m_{e}}+\frac{\hbar e}{m_{h}}\right] \cdot B
$$

In this case a stronger spectral shift of about $0.65 \mathrm{meV} / T$ is found.

A summary of the experimental data obtained from the $\mathrm{ZnCdSe} / \mathrm{ZnSe}$ MQWs is displayed in Figure 34 (Cingolani et al., 1994e). Clearly two classes of results can be identified: excitonic shift for the deepest quantum wells, with binding energies as high as $35 \mathrm{meV}$, and Landau shift for the other samples with binding energies smaller than $30 \mathrm{meV}$. These data demonstrate that both excitonic or free-carrier lasing can occur in these quantum wells.

Shallow quantum wells exhibit a free-carrier behavior. The stimulated emission always occurs at carrier densities corresponding to bleached exciton features in the pump and probe spectra, though smaller than expected from Eq. (16), corresponding to the free-carrier-rich side of the phase diagram. The excitonic stimulated emission occurs in deep quantum wells at carrier densities falling in the exciton-rich side of the phase diagram, as expected from the strong stability of the elementary excitation. In these samples the excitonic recombination is observed up to the strongest injection densities, when the excitonic features have been bleached in the pump and probe spectra. This is probably due to rather high $\mathrm{Cd}$ content of these samples causing appreciable compositional fluctuations $( \pm 1 \%)$ leading to exciton localization. This phenomenon is expected to raise considerably the screening threshold of the exciton, and is not accounted for in the calculation of the exciton/free-carrier gas phase diagram.

Similar results are obtained from the $\mathrm{ZnSe} / \mathrm{ZnSSe}$ superlattices. In Figure 35 we display a summary of the magnetophotoluminescence experiments performed on a representative $\mathrm{ZnSSe} / \mathrm{ZnSe}$ sample $(5 \mathrm{~nm} / 5 \mathrm{~nm}$ superlattice) (Cingolani et al., 1996b). The symbols represent the energy positions of the 


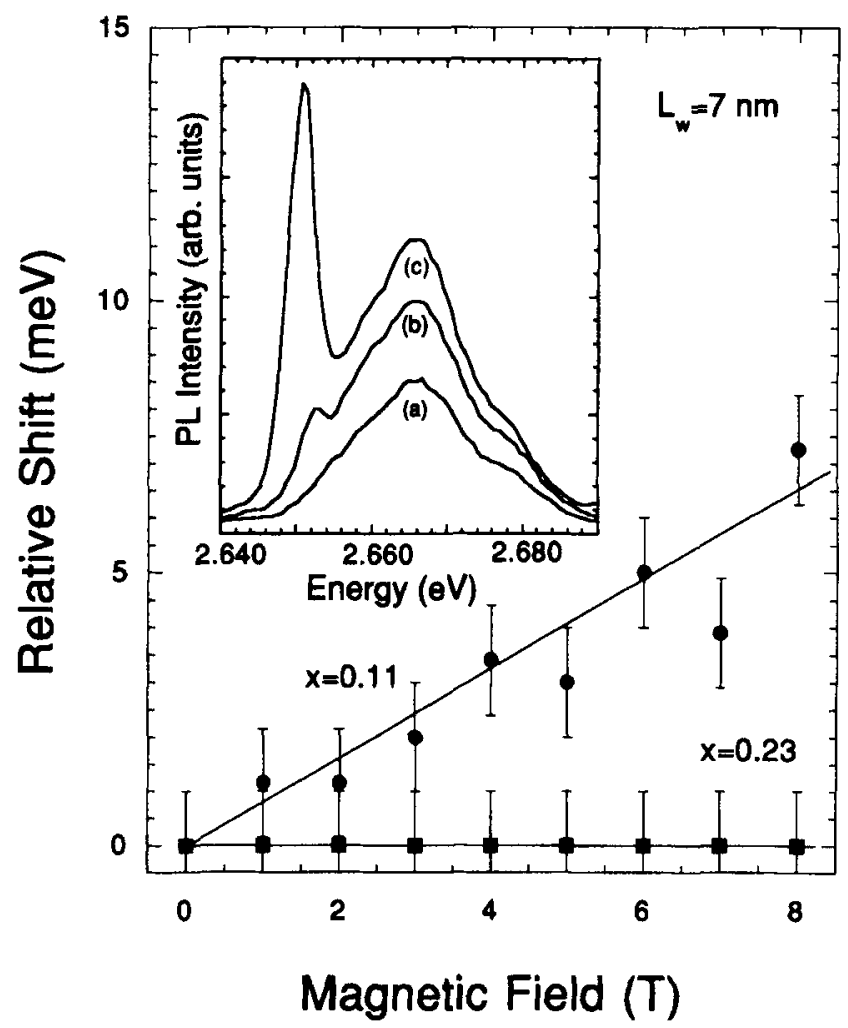

Fig. 34. Shift of the stimulated emission in magnetic field for a deep $(z=0.23)$ and a shallow $(x=0.11) \mathrm{Zn}_{(1-x)} \mathrm{Cd}_{x} \mathrm{Se} / \mathrm{ZnSe}$ multiple quantum well heterostructure at $4 \mathrm{~K}$. The well width is $L_{w}=7 \mathrm{~nm}$. Inset: Stimulated emission spectra around the threshold. (a) $1 \mathrm{~kW} \mathrm{~cm}^{-2}$, (b) $4 \mathrm{~kW} \mathrm{~cm}{ }^{-2}$, and (c) $10 \mathrm{~kW} \mathrm{~cm}^{-2}$.

various features displayed in Figure 14. The heavy-hole exciton measured by PLE (HH, empty squares), the strong localized exciton (LE, empty dots), and the first phonon replica ( $\mathrm{PR}_{1}$, empty triangles) measured under lowpower continuous-wave excitation exhibit an almost constant energy position up to magnetic field of $8 \mathrm{~T}$. This is consistent with the small excitonic diamagnetic shift predicted by Eq. (30) (continuous lines). Actually an even smaller diamagnetic shift should be observed for localized excitons. However, this is below the resolution limit of the equipment used in this experiment. The spontaneous emission band measured under strong excitation, just below threshold (labeled SP in Fig. 35) also exhibits no detectable shift with increasing the magnetic field (downwards empty triangles). This is a clear signature of excitonic recombination, even though the photo- 


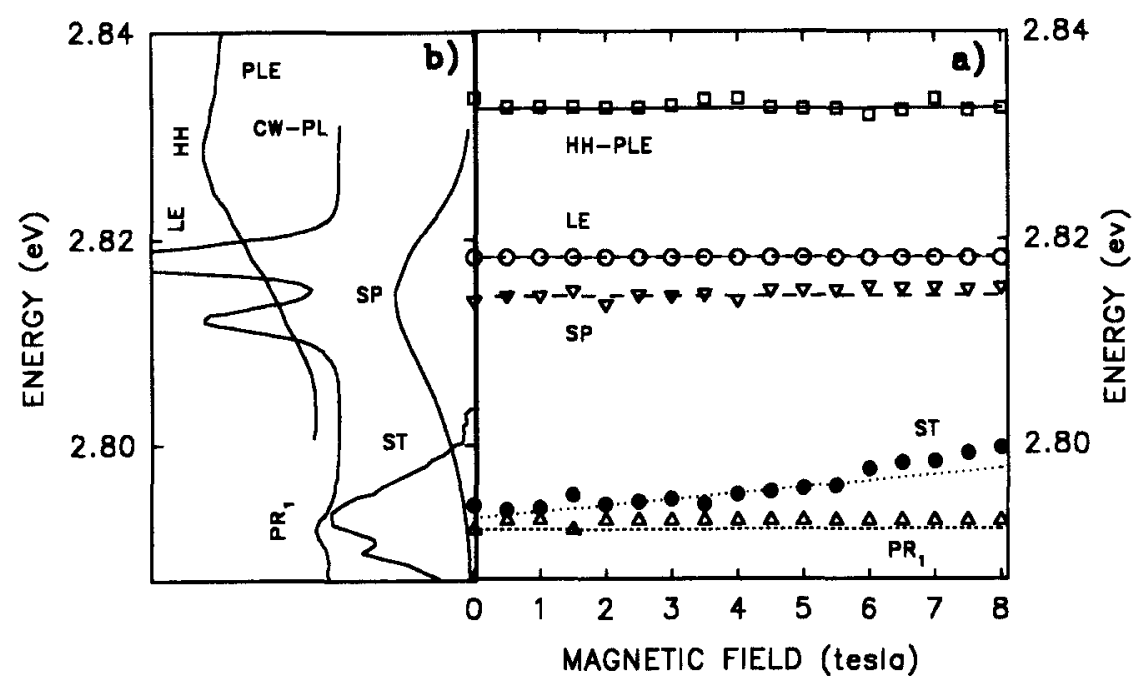

FIG. 35. (a) Magnetoluminescence shift of the main emission lines of a symmetric $\mathrm{ZnS}_{0.18} \mathrm{Se}_{0.82} / \mathrm{ZnSe}$ superlattice at $4 \mathrm{~K}$. Empty symbols indicate excitonic processes: the heavy-hole exciton band in photoluminescence excitation (HH-PLE, empty squares), the localized exciton continuous wave luminescence ( $L E$, empty circles) and its phonon replica (PR and upwards empty triangles), and the spontaneous emission obtained under strong pulsed excitation just below threshold (SP, downwards triangles). The full symbols indicate free-carrier processes associated with the stimulated emission (ST, full circles).

generated carrier density has been raised in the $10^{11} \mathrm{~cm}^{-2}$ range. On the contrary, the stimulated emission band (ST and full dots in Fig. 35) is found to shift linearly with the magnetic field, consistent with Eq (31) (dotted line), clearly indicating that lasing occurs through electron-hole recombination in our $\mathrm{ZnSSe} / \mathrm{ZnSe}$ superlattices. We should mention that the stimulated emission band in Figure 35 has been measured under strong optical pumping of intensity far above the stimulation threshold $\left(I \sim 10 I_{0}\right)$. The energy position is therefore strongly red-shifted with respect to the $\mathrm{HH}$ peaks shown in Figure 14 (in this case it is even coincident with the phonon replica $\mathbf{P R}_{1}$, resulting in possibly erroneous attributions). However, the intensity-dependent measurements confirmed the Landau shift of the stimulated emission band at any injection level above the threshold (with the zero-field spectral position of the lasing line in the range 2.79-2.81 eV). Only at excitation intensities very close to $I_{0}$, the stimulated emission line disappeared and the spontaneous emission band (SP) showed up a clear diamagnetic behavior (empty downwards triangles). 


\section{Conclusions}

In conclusion, we have overviewed the main linear and nonlinear optical properties of excitons in $\mathrm{ZnSe}$-based quantum wells. The fundamental importance of strongly bound excitons in terms of polariton properties, time dynamics, and oscillator strength has been addressed through the discussion of recent spectroscopic experiments. The role of excitons in the operation of optoelectronic devices operating in the blue-green region has been emphasized in the discusion of the nonlinear optical properties. The unique combination of large exciton binding energy and reduced screening and phonon coupling occurring in quantum wells permits the observation of novel phenomena connected with the coexistence of the exciton gas and the free-carrier gas at high density. Besides their fundamental importance, these phenomena are found to play an important role in the physics of blue-green lasers and modulators.

\section{ACKNOWLEDGMENTS}

This work is the outcome of a collaboration between different groups. The author wishes to thank the group of optoelectronics at the Material Science Department of the University of Lecce: L. Calcagnile, G. Coli', M. DeVittorio, A. Cola, D. Greco, P. V. Giugno, P. Prete, and R. Rinaldi for the collaborative work on the spectroscopy and transport experiments. $M$. Lomascolo, M. DiDio, and L. Tapfer of the CNRSM laboratory (Brindisi, Italy) are gratefully acknowledged for their collaboration on the timeresolved luminescence experiments and structural analysis. Special thanks to A. Franciosi, L. Sorba, and L. Vanzetti at the TASC laboratory (Trieste, Italy) for their continuous collaboration and for the MBE growth of the high-quality $\mathrm{ZnCdSe} / \mathrm{ZnSe}$ heterostructures. The author is also indebted to I. Suemune (Hokkaido University, Japan) for supplying the MOCVDgrown $\mathrm{ZnSe} / \mathrm{ZnSSe}$ superlattices.

\section{REFERENCES}

Alonso, R. G., Parks, C., Ramdas, A., Luo, H., Samarth, N., Furdyna, J. K., and Ram-Mohan, L. R. (1992). Phys. Rev. B45, 1181.

Andreani, L. C., D'Andrea, A., and Del Sole, R. (1992). Physics Lett. A168, 451.

Baranovskii, S. D., Doerr, U., Thomas, P., Naumov, A., and Gebhardt, W. (1993). Phys. Rev. B48, 17149.

Bastard, G., Mendez, E. E., Chang, L. L., and Esaki, L. (1982). Phys. Rev. B26, 1974.

Bastard, G. (1991). Wave Mechanics Applied to Semiconductor Heterostructures. Editions de Physique, Les Ulis, Paris. 
Bertho, D., and Jouanin, C. (1993). Phys. Rev. B47, 2184.

Bratina, G., Nicolini, R., Sorba, L., Vanzetti, L., Mula, G., Yu, X., and Franciosi, A. (1993). J. Cryst. Growth 127, 387.

Calcagnile, L., Lomascolo, M., Rinaldi, R., Prete, P., Cingolani, R., Vanzetti, L., Bonanni, A., Bassani, F., Sorba, L., and Franciosi, A. 22nd International Conference on the Physics of Semiconductors August 15-19, 1994, Vancouver, Canada.

Calcagnile, L., Rinaldi, R., Prete, P., Stevens, C. J., Cingolani, R., Vanzetti, L., Sorba, L., and Franciosi, A. (1996). Phys. Rev. B54, 17248.

Campi, D., and Coriasso, C. (1995). Phys. Rev. B51, 7985.

Chemla, D. S., Miller, D. A. B., Smith, P. W., Gossard, A. C., and Wiegmann, W. (1984). IEEE J. Quantum El. QE-20, 265.

Chung, H. Y. A., Uhle, N., and Tschudi, T. (1993). Appl. Phys. Rev. 63, 1378.

Cingolani, R., Stolz, W., Ploog, K. (1990). Phys. Rev. B40, 2950.

Cingolani, R., LaRocca, G. C., Kalt, H., Ploog, K., Potemsky, M., and Maan, J. C. (1991). Phys. Rev. B43, 9662.

Cingolani, R., and Ploog, K. (1991). Adv. Phys. 40, 535.

Cingolani, R., Rinaldi, R., Calcagnile, L., Prete, P., Sciacovelli, P., Tapfer, L., Vanzetti, L., Mula, G., Bassani, F., Sorba, L., and Franciosi, A. (1994a). Phys. Rev. B49, 16769.

Cingolani, R. Lomascolo, M., Lovergine, N., Dabbicco, M., Ferrara, M., and Suemune, I. (1994b). Appl. Phys. Rev. 64, 2439.

Cingolani, R., Di Dio, M., Lomascolo, M., Rinaldi, R., Prete, P., Vasanelli, L., Vanzetti, L., Bassani, F., Bonnani, A., Sorba, L., and Franciosi, A. (1994c). Phys. Rev. B50, 12179.

Cingolani, R., de Vittorio, M., DiDio, M., Lomascolo, M., Cola, A., Vasanelli, L., Vanzetti, L., Sorba, L., and Franciosi, A. (1994d). Superlatt. Microstr. 16, 363.

Cingolani, R., Calcagnile, L., Franciosi, A., Sorba, L., and Vanzetti, L. (1994e). SPIE II-VI Blue Green Laser Diodes 112, 2346.

Cingolani, R., Prete, P. Greco, D., Giugno, P. V., Lomascolo, M., Rinaldi, R., Calcagnile, L. Vanzetti, L., Sorba, L., and Franciosi, A. (1995). Phys. Rev. B51, 5176.

Cingolani, R., Calcagnile, L., Colǐ, G., Rinaldi, R., Lomascolo, M., Didio, M., Franciosi, A., Vanzetti, L., La Rocca, G. C., and Campi, D. (1996a). J. Opt. Soc. Am. B13, 1268.

Cingolani, R., Colĭ, G., Calcagnile, L., Convertino, A. L., Lomascolo, M., Didio, M., and Suemune, I. (1996). Phys. Rev. B54, 15 December.

Citrin, D. S. (1994). Phys. Rev. B50, 5497.

Dabbicco, M., Cingolani, R., Ferrara, M., Suemune, I., and Kuroda, Y. (1992). Appl. Phys. Lett. 72, 4969.

D'Andrea, A., and Del Sole, R. (1990). Phys. Rev. B41, 1413.

Ding, J., Jeon, H., Nurmikko, A. V., Luo, H., Samarth, N., and Furdyna, J. K. (1990a). Appl. Phys. Lett. 57, 2756.

Ding, J., Pelekanos, N., Nurmikko, A. V., Luo, H., Samarth, N., and Furdyna, J. K. (1990b). Appl. Phys. Lett. 57, 2885.

Ding, J., Jeon, H., Ishihara, T. Hagerott, M., Nurmikko, A. V., Luo, H., Samarth, N., and Furdyna, J. (1992). Phys. Rev. Lett. 69, 1707.

Ding, J., Hagerott, M., Ishihara, T., Jeon, H., and Nurmikko, A. V. (1993). Phys. Rev. B47, 10528.

Fu, Q., Lee, D., Mysyrowicz, A., Nurmikko, A. V., Gunshor, R. L., and Kolodziejski, L. A. (1988). Phys. Rev. B37, 8791.

Fujii, Y., Suemune, I., and Fujimoto, M. (1994). Jpn. A. Appl. Phys. 33, 840.

Galbraith, I. (1992). Phys. Rev. B45, 6950.

Givgno, P. V., de Vittorio, M., Rinaldi, R., Cingolani, R., Quaranta, F., Vanzetti, L., Sorba, L., and Franciosi, A. (1996). Phys. Rev. BS4, 16934.

Gil, B., Cloitre, T., DiBlasio, M., Bigenwald, P., Aigouy, L., Briot, N., Briot, O., Bouchara, D., Aulombard, R. L., and Calas, J. (1994). Phys. Rev. B50, 18231.

Gorczyca, I., and Christensen, N. E. (1993). Phys. Rev. B48, 17202.

Gowar, J. (1984). Optical Communication Systems. Prentice Hall, New York. Chapter 8. 
Grahan, H. T., Fisher, A., and Ploog, K. (1992). Appl. Phys. Lett. 61, 2211.

Greco, D., Cingolani, R., D’Andrea, A., Tommasini, N., Vanzetti, L., and Franciosi, A. (1996). Phys. Rev. B54, 16998.

Gurioli, M., Martinez-Pastor, J., Colocci, M., Deparis, C., Chastaingt, B., and Massies, J. (1992). Phys. Rev. B46, 6922.

Gutowsky, J., Diessel, A., Neukirch, U., Weckendrup, D., Beher, T., Jobst, B., and Hommel, D. (1995). Phys. Stat. Sol. B187, 423.

Haug, H., and Schmitt-Rink, S. (1985). J. Opt. Soc. Am. B2, 1135.

Hohnoki, S., Katayama, S., and Hasegawa, A. (1994). Solid State Commun. 89, 41.

Hopfield, J. J., and Thomas, D. G. (1963). Phys. Rev. 132, 563.

Hwang, S. J., Sham, W., Song, J. J., Zhu, Z. Q., and Tao, T. (1994). Appl. Phys. Lett. 64, 2267.

Kawakami, Y., Wang, S. Y., Simpson, J., Hauksson, I., Adams, S. J. A., Stewart, H., Cavenett, B. C., and Prior, K. A. (1993a). Physica B185, 508.

Kawakami, Y., Hauksson, I., Stewart, H., Simpson, J., Galbraith, I., Prior, K. A., and Cavenett, B. C. (1993b). Phys. Rev, B B48, 11994.

Klingshirn, C., and Haug, H. (1981). Phys. Report 70, 316.

Kozlov, V., Kelkar, P., Nurmikko, A. V., Chu, C.-C., Grillo, D. L., Han, J., Hua, C. G., and Gunshor, R. L. (1996). Phys. Rev. B53, 10837.

Kreller, F., Lowisch, M., Puls, J., and Henneberger, F. (1995). Phys. Rev. Lett. 75, 2420.

Kuroda, Y., Suemune, I., Fujimoto, M., and Fuji, A. (1992a). J. Appl. Phys. 72, 3029.

Kuroda, Y., Suemune, I., Fuji, A., and Fujimoto, M. (1992b). Appl. Phys. Lett. 61, 1182.

Kuroda, T. Inoue, K., Suemune, I., and Minami, F. (1994). Proc. 22nd Int. Conf. Physics of Semiconductors, Vol. II. Vancouver, 1388.

Landolt-Bornstein. (1982). Numerical Data and Functional Relationship in Science and Technology, ed. O. Madelung. Springer, Berlin. Gp. III, Vol. 17a.

Lasher, G., and Stern, F. (1964). Phys. Rev. A133, 553.

Lawaetz, P. (1971). Phys. Rev. B4, 3460.

Leavitt, R. P., and Bradshaw, J. L. (1991). Appl. Phys. Lett. 59, 2433.

Lee, J., Koteles, E., and Vassel, M. O. (1986). Phys. Rev. B33, 5512.

Liaci, F., Bigenwald, P., Briot, O., Gil, B., Briot, N., Cloitre, T., and Aulombard, P. L. (1995). Phys. Rev. B51, 4699.

Lippens, P. E., and Lanoo, M. (1989). Phys. Rev. B39, 10935.

Livescu, G., Miller, D. A. B., Chemla, D. S., Ramaswamy, M., Chang, T. Y., Sauer, N., Gossard, A. C., and English, J. H. (1988). IEEE J, Quant. Electron. QE-24, 1677.

Lomascolo, M., Didio, M., Greco, D., Calcagnile, L., Cingolani, R., Vanzetti, L., Sorba, L., and Franciosi, A. (1996). Appl. Phys. Lett. 69, 1145.

Lomascolo, M., Li, G. H., Syassen, K., Cingolani, R., and Suemune, I. (1994). Phys. Rev. B50, 14635 .

Lozykowsky, H. J., and Shastri, V. K. (1992). J. Appl. Phys. 69, 3235.

Marquadt, E., Opitz, B., Scholl, M., and Heuken, M. (1994). J. Appl. Phys. 75, 8022.

Marshal, T., Colak, S., and Cammack, D. (1989). J. Appl. Phys. 66, 1753.

Masumoto, Y., Matsuura, M., Tarucha, S., and Okamoto, H. (1985). Phys. Rev. B32, 4275.

Michler, P., Hangleiter, A., Moser, M., Geiger, M., and Scholz, F. (1992). Phys. Rev. B46, 7280.

Miller, D. A. B., Chemla, D. S., Damen, T. C., Gossard, A. C., Wiegmann, W., Wood, T. H., and Burrus, C. A. (1985). Phys. Rev. B32, 1043.

Mohammed, K., Olego, D. J., Newbury, P., Cammack, D. A., Dalby, R., and Cornellisen, H. (1987). Appl. Phys. Lett. 50, 1820.

Moss, T. S., Burrell, G. J., and Ellis, B. (1973). Semiconductor Optoelectronics. Butterworth and Co., London. Chapter 5.

Neukirch, U. Weckendrup, D., Gutowski, J., Hommel, D., and Landwher, G. (1994). J. Cryst. Growth 138, 861.

Pelekanos, N. T., Haas, H., Magnea, N., Mariette, H., and Asiela, A. W. (1992a). Appl. Phys. Lett. 61, 3154. 
Pelekanos, N. T., Ding, J., Hagerott, M., Nurmikko, A. V., Luo, H., Samarth, N., and Furdyna, J. K. (1992b). Phys. Rev. B45, 6037.

Pellegrini, V., Atanasov, R., Tredicucci, A., Beltram, F., Amzulini, C., Sorba, L., Vanzetti, L., and Franciosi, A. (1995). Phys. Rev. B51, 5176.

Pollak, F. H., and Cardona, M. (1968). Phys. Rev. 172, 816.

Quiroga, L., Rodriguez, F. J., Camacho, A., and Tejedor, C. (1990). Phys. Rev. B42, 11198.

Ren, S.-F., Gu, Z.-Q., and Chung, Y.-C. (1994). Phys. Rev. B49, 7569.

Rudin, S., Reinecke, T. L., and Segal, B. (1990). Phys. Rev. B42, 11218.

Schmitt-Rink, S., Chemla, D. S., and Miller, D. A. B. (1985). Phys. Rev. B32, 6601.

Schmitt-Rink, S., Chemla, D. S., and Miller, D. A. B. (1989). Advances in Physics 38, 89.

Shahzad, K., Olego, D. J., and Van de Walle, C. G. (1988). Phys. Rev. B38, 1417.

Shan, W., Hwang, S. J., Hays, J. M., Song, J. J., Zhu, Z. Q., and Yao, T. (1993). J. Appl. Phys. 74, 5699.

Shen, A., Wang, H., Wang, Z., and Lu, S. (1992). Appl. Phys. Lett. 60, 2640.

Singh, R. K., and Singh, S. (1987). Phys. Stat. Sol. B140, 407.

Stevens, C. J., Taylor, R. A., Ryan, J. F., Cingolani, R., Dabbicco, M., Ferrara, M., and Suemune, I. (1994). Semicond. Sci. Technol. 9, 762.

Suemune, I. (1993). Appl. Phys. Lett. 63, 2612.

Suemune, I, and Yamanishi, M. (1993) New Functionality Materials, Volume A: Optical and Quantum-structural Properties of Semiconductors, ed T. Tsuruta, M. Doyama, M. Seno, and S. Fujita. Elsevier Science Publ., Amsterdam.

Suemune, I., Fujii, Y., and Fujimoto, M. (1994). J. Cryst. Growth 138, 750.

Sugawara, M. (1992). J. Appl. Phys. 71, 277.

Tamargo, M. C., Brasil, M. J. S. P., Nahory, R. E., Martin, R. J., Waever, A. L., and Gilchrist, H. L. (1991). Semicond. Sci. Technol. 6, A8.

Tapfer, L., and Ploog, K. (1986). Phys. Rev. B33, 5565.

Thomas, R. J., Chandrasekhar, H. R., Chandrasekhar, M., Samarth, N., Luo, H., and Furdyna, J. (1992). Phys. Rev. B45, 9505.

Tokizaki, T., Sakai, H., Nakamura, A. Manabe, Y., Hayashi, S., and Mitsuyu, T. (1994). Proc. 22nd Int. Conf. Physics Semiconductors, Vol. II. Vancouver, 1532.

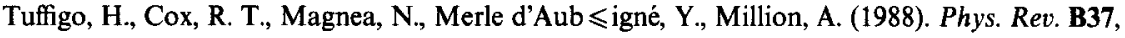
4310.

Tsutsumi, T., Yen, J. Y., Suoma, I., and Oka, Y. (1994). Superlatt. Microstr. 16, 000.

Ves, S., Schwartz, U., Christenses, N. E., Syassen, K., and Cardona, M. (1990). Phys. Rev. B42, 9113.

Wang, L. and Simmons, J. (1995). Appl. Phys. Lett. 67, 1450.

Wang, S. Y., Simpson, J., Stewart, H., Adams, S. J. A., Hauksson, I., Kawakami, Y., Taghizadeh, M. R., Prior, K. A., and Cavenett, B. C. (1993a). Physica B185, 508.

Wang, S. Y., Horsburgh, G., Thompson, P., Hauksson, I., Mullins, J. T., Prior, K. A., and Cavenett, B. C. (1993b). Appl. Phys. Lett. 63, 857.

Wang, S. Y., Thompson, P., Horsburgh, G., Mullins, J. T., Livingstone, M., Galbraith, I., Prior, K. A., and Cavenett, B. C. (1994). Proc. 22nd Int. Conference Physics of Semiconductors, Vol. 2. 1536.

Wu, J. W., and Nurmikko, A. V. (1988). Phys. Rev., B38, 1504.

Yamada, Y., Masumoto, Y., Taguchi, T., and Takemura, K. (1991). Phys. Rev. B44, 1801.

Yamada, Y., Mishina, T., Masumoto, Y., Kawakami, Y., Suda, J., and Fujita, S. (1995). Phys. Rev. B52, R2289.

Yang, F., Henderson, B., and O'Donnel, K. P. (1993). Solid State Commun. 88, 687.

Yao, F., Fujimoto, M., Chang, S. K., and Tanino, H. (1991). J. Cryst. Growth 111, 823.

Yokogawa, T., Ishikawa, T., Merz, J. L., and Taguchi, T. Y. (1994). J. Appl. Phys. 75, 2189.

Yokuda, Y., Abe, Y., and Tsukada, N. (1994). J. Appl. Phys. 75, 1620.

Young, P. M., Runge, E., Ziegler, M., and Ehrenreich, H. (1994). Phys. Rev. B49, 7424.

Zhu, W., Yoshihara, H., Takebayashi, K., and Yao, T. (1993). Appl. Phys. Lett. 63, 1678. 